Article

\title{
Synthesis, Docking, 3-D-Qsar, and Biological Assays of Novel Indole Derivatives Targeting Serotonin Transporter, Dopamine D2 Receptor, and Mao-A Enzyme: In the Pursuit for Potential Multitarget Directed Ligands
}

\author{
Christopher Cerda-Cavieres ${ }^{1}$, Gabriel Quiroz ${ }^{2}$, Patricio Iturriaga-Vásquez ${ }^{3}{ }^{[}$, \\ Julio Rodríguez-Lavado ${ }^{1}$, Jazmín Alarcón-Espósito ${ }^{1}$, Claudio Saitz ${ }^{1}$, Carlos D. Pessoa-Mahana ${ }^{4}$, \\ Hery Chung ${ }^{4}\left(\mathbb{D}\right.$, Ramiro Araya-Maturana ${ }^{5} \mathbb{D}$, Jaime Mella-Raipán ${ }^{6,7}$, David Cabezas ${ }^{6}$, \\ Claudia Ojeda-Gómez ${ }^{8}$, Miguel Reyes-Parada $9,10, * \mathbb{D}$ and Hernán Pessoa-Mahana ${ }^{1, *}$ \\ 1 Departamento de Química Orgánica y Fisicoquímica, Facultad de Ciencias Químicas y Farmacéuticas, \\ Universidad de Chile, Casilla 233, Santiago 8380494, Chile; cdc.cavieres@gmail.com (C.C.-C.); \\ julio.rodriguez@ciq.uchile.cl (J.R.-L.); jazmin.alarc@gmail.com (J.A.-E.); clsaitz@ciq.uchile.cl (C.S.) \\ 2 Programa de Doctorado en Farmacología, Universidad de Chile, Santiago 8380453, Chile; \\ gquirozn@ciq.uchile.cl \\ 3 Departamento de Ciencias Químicas y Recursos Naturales, Facultad de Ingeniería y Ciencias, Universidad \\ de la Frontera, Temuco 4811230, Chile; patricio.iturriaga@ufrontera.cl \\ 4 Departamento de Farmacia, Facultad de Química, Pontificia Universidad Católica de Chile, Santiago \\ 7820244, Chile; cpessoa@puc.cl (C.D.P.-M.); chung.hery@gmail.com (H.C.) \\ 5 Instituto de Química y Recursos Naturales, Universidad de Talca, Talca 3460000, Chile; raraya@utalca.cl \\ 6 Instituto de Química y Bioquímica, Facultad de Ciencias, Universidad de Valparaíso, Av. Gran Bretaña 1111, \\ Valparaíso 2360102, Chile; jaime.mella@uv.cl (J.M.-R.); jaime.mella@uc.cl (D.C.) \\ 7 Centro de Investigación Farmacopea Chilena (CIFAR), Universidad de Valparaíso, Av. Gran Bretaña 1111, \\ Valparaíso 2360102, Chile \\ 8 Colegio Instituto San Martín, Hermanos Maristas, Curicó 3341965, Chile; claudia-ojeda@gmail.com \\ 9 Centro de Investigación Biomédica y Aplicada (CIBAP), Escuela de Medicina, Facultad de Ciencias Médicas, \\ Universidad de Santiago de Chile, Santiago 9170002, Chile \\ 10 Facultad de Ciencias de la Salud, Universidad Autónoma de Chile, Talca 3467987, Chile \\ * Correspondence: miguel.reyes@usach.cl (M.R.-P.); hpessoa@ciq.uchile.cl (H.P.-M.)
}

Academic Editors: Tomasz Plech and Marta Andres-Mach

Received: 27 August 2020; Accepted: 3 October 2020; Published: 10 October 2020

Abstract: A series of 27 compounds of general structure 2,3-dihydro-benzo[1,4]oxazin-4-yl)-2\{4-[3-(1H-3indolyl)-propyl]-1-piperazinyl\}-ethanamides, Series I: 7(a-o) and (2-\{4-[3-(1H-3-indolyl) -propyl]-1-piperazinyl\}-acetylamine)- $N$-(2-morfolin-4-yl-ethyl)-fluorinated benzamides Series II: 13(a-1) were synthesized and evaluated as novel multitarget ligands towards dopamine $D_{2}$ receptor, serotonin transporter (SERT), and monoamine oxidase-A (MAO-A) directed to the management of major depressive disorder (MDD). All the assayed compounds showed affinity for SERT in the nanomolar range, with five of them displaying Ki values from 5 to $10 \mathrm{nM}$. Compounds $7 \mathrm{k}, \mathrm{Ki}=5.63 \pm 0.82 \mathrm{nM}$, and $13 \mathrm{c}, \mathrm{Ki}=6.85 \pm 0.19 \mathrm{nM}$, showed the highest potencies. The affinities for $\mathrm{D}_{2}$ ranged from micro to nanomolar, while MAO-A inhibition was more discrete. Nevertheless, compounds $7 \mathbf{m}$ and $\mathbf{7 n}$ showed affinities for the $\mathrm{D}_{2}$ receptor in the nanomolar range ( $7 \mathrm{n}: \mathrm{Ki}=307 \pm 6 \mathrm{nM}$ and $7 \mathrm{~m}: \mathrm{Ki}=593 \pm 62 \mathrm{nM}$ ). Compound $\mathbf{7 n}$ was the only derivative displaying comparable affinities for SERT and $\mathrm{D}_{2}$ receptor $\left(\mathrm{D}_{2} / \mathrm{SERT}\right.$ ratio $\left.=3.6\right)$ and could be considered as a multitarget lead for further optimization. In addition, docking studies aimed to rationalize the molecular interactions and binding modes of the designed compounds in the most relevant protein targets were carried out. Furthermore, in order to obtain information on the structure-activity relationship of the synthesized series, a 3-D-QSAR CoMFA and CoMSIA study was conducted and 
validated internally and externally $\left(\mathrm{q}^{2}=0.625,0.523\right.$ for CoMFA and CoMSIA and $\mathrm{r}^{2}$ ncv $=0.967$, 0.959 for CoMFA and CoMSIA, respectively).

Keywords: polypharmacology; $\mathrm{SERT}$; dopamine $\mathrm{D}_{2}$ receptor; 3-indolylpropylpiperazines; multitarget; docking; QSAR

\section{Introduction}

Major depressive disorder (MDD) is a common, chronic, recurring, heterogeneous, and potentially life-threatening disease affecting up to $20 \%$ of the world population, according to World Health Organization [1]. MDD is triggered by a complex pattern of genetic, epigenetic, and environmental factors and, despite its prevalence, it is not being properly treated [2-4]. As a result, such a devastating disorder is enormously costing, economically, socially, and individually $[5,6]$.

Since the monoamine hypothesis of depression was developed in the 1950s [7], antidepressant drug discovery has been a very active research field [8], although MDD's underlying mechanism and neurological basis are not yet fully understood [9-11]. The global antidepressants market is expected to grow from $\$ 14.3$ billion in 2019 to about $\$ 28.6$ billion in 2020 as mental health issues are expected to surge because of the Covid-19 pandemic making an impact on the global economy.

Thus far, the vast majority of marketed antidepressants act through the serotoninergic and norepinephrinergic systems, elevating synaptic levels of the corresponding neurotransmitters by blocking the monoamine transporters [12-15]. Many adverse effects associated with first-generation antidepressants (mainly tricyclic and monoamine oxidase inhibitors, $-\mathrm{MAOi}-$ ) were partially overcome by the arrival of second-generation antidepressants, including selective serotonin reuptake inhibitors (SSRIs), norepinephrine reuptake inhibitors (NRIs), and dual antidepressants (SNRIs), with the latter being advantageous as they can treat a wide range of symptoms [16-19]. However, much less approved antidepressants do target the dopamine system, notwithstanding dopamine has also been implicated in the pathophysiology of depression [20].

Inside the central nervous system (CNS), dopamine plays pivotal roles in executive functions, motor control, emotion, cognition, reinforcement, and reward, among others [21-23]. Its dysfunction is related to the pathophysiology of several CNS disorders, such as Parkinson's disease, multiple sclerosis, schizophrenia, drug addiction, and MDD [24-26]. Even though depression has been historically associated with misregulation within the serotonin/norepinephrine system, in the last years, an increasing number of voices have been raised supporting that dopamine system dysfunction strings along with it $[24,27,28]$.

Due to the aforementioned causes, MDD is nowadays recognized as a complex dynamic system [29] endowed with a complex pathophysiology. For this reason, depression symptoms and its underlying causes are more likely to be treated by a multitarget approach, by using promiscuous drugs also defined as multi-target directed ligands (MTDLs): A single drug molecule that selectively targets multiple receptors, so that different pathways conducting to the disease can be modified by using a single molecule [20,30-32].

Indole derivatives have always been recognized as serotoninergic modulators, given their related structural connection. Furthermore, in our research group, we have extensive experience in the synthesis of indolylpropyl-piperazine derivatives, which have been successfully employed by us [33-37] and other groups $[38,39]$ as very potent serotonin transporter (SERT) ligands. On the other hand, molecular structures containing morpholine [40] or benzoxazinone [41-45] cores have been reported as MAOi or dopamine $\mathrm{D}_{2}$ receptor modulators, respectively. In the light of such a background, we decided to conduct an exploratory study by merging the aforementioned indolylpropyl-piperazines and morpholine/benzoxazinone units. Herein, we describe the synthesis, docking, QSAR, and biological 
evaluation of two series of molecular hybrids targeting SERT, MAO-A, and $\mathrm{D}_{2}$ receptor in the pursuit of promising multi-target leads for potential MDD treatment.

\section{Results and Discussion}

\subsection{Chemistry Series I}

2,3-Dihydro-benzo[b][1,4] oxazin-4-yl)-2-\{4-[3-(1H-3-indolyl)-propyl]-1-piperazinyl $\}$ ethanamide derivatives $7 \mathbf{a}-\mathbf{o}$

The synthetic approach for the preparation of the target compounds $7 \mathbf{a}-\mathbf{o}$ is outlined below. The reaction took place between piperazine benzoxazine derivatives $\mathbf{6}(\mathbf{a}-\mathbf{c})$ obtained in a six-step sequence and $82-95 \%$ yields (Scheme 1), with 3-indolyl tosylates $1 \mathbf{a}-\mathbf{c}(\mathrm{R}=\mathrm{H}, \mathrm{F}, \mathrm{Br})$ obtained by reported literature procedures $[37,46]$ to give nine final compounds $7(\mathbf{a}, \mathbf{b}, \mathbf{c}, \mathbf{g}, \mathbf{h}, \mathbf{i}, \mathbf{m}, \mathbf{n}, \mathbf{o})$ in a $42-89 \%$ yield (Scheme 2).

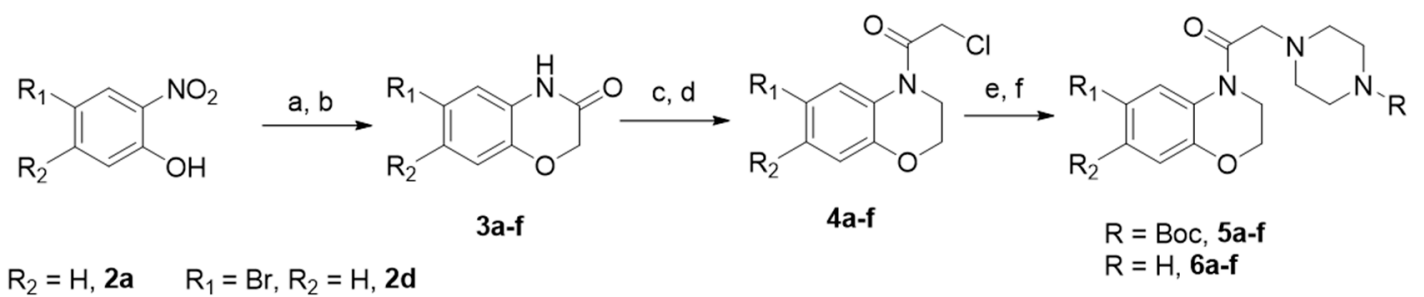

$R_{1}=R_{2}=H, 2 a \quad R_{1}=B r, R_{2}=H, 2 d$

$R_{1}=H, R_{2}=F, 2 b \quad R_{1}=C l, R_{2}=F, 2 e$

$R_{1}=F, R_{2}=H, 2 c \quad R_{1}=O M e, R_{2}=H, 2 f$

Scheme 1. Synthesis of piperazinylbenzoxazine derivatives $6(\mathbf{a}-\mathbf{c})$. Reagents and experimental conditions.

(a) $\mathrm{Fe}^{\circ} /\left(\mathrm{H}_{2} \mathrm{O} / \mathrm{EtOH} / \mathrm{CH}_{3} \mathrm{COOH}\right)$ 1:1:1/60 ${ }^{\circ} \mathrm{C}$; (b) 2-chloroacetyl chloride, $\mathrm{CH}_{3} \mathrm{CN}, \mathrm{K}_{2} \mathrm{CO}_{3}, 80{ }^{\circ} \mathrm{C}$;

(c) $\mathrm{THF} / \mathrm{LiAlH}_{4} / \mathrm{N}_{2} /{ }^{\circ} \mathrm{C}$; (d) 2-chloroacetyl chloride-THF, $0{ }^{\circ} \mathrm{C}$; (e) $\mathrm{N}$-Boc-piperazine, $\mathrm{CH}_{3} \mathrm{CN}_{2} \mathrm{~K}_{2} \mathrm{CO}_{3}$, $80{ }^{\circ} \mathrm{C}$; (f) TFA- $\mathrm{CH}_{2} \mathrm{Cl}_{2}, 0{ }^{\circ} \mathrm{C}$.

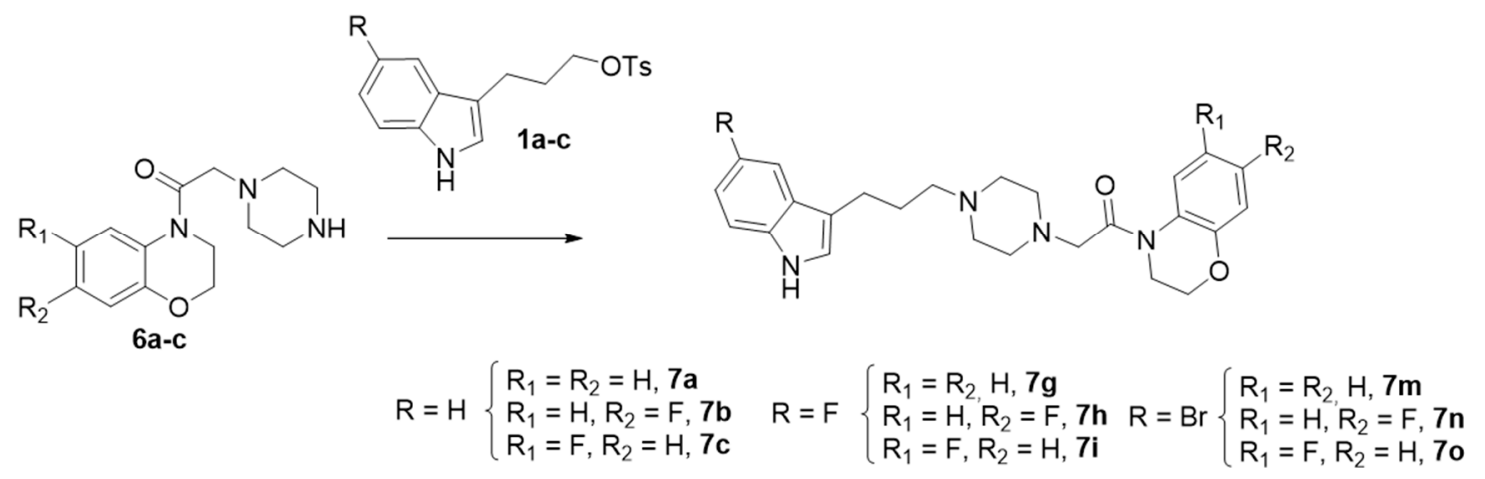

Scheme 2. Synthesis of Series I. Reagents and conditions: $\mathrm{K}_{2} \mathrm{CO}_{3}, \mathrm{CH}_{3} \mathrm{CN}, 80{ }^{\circ} \mathrm{C}$, Yield $(42-89 \%)$.

The synthesis of the derivatives $\mathbf{7} \mathbf{d}-\mathbf{f}$ and $\mathbf{7 j} \mathbf{j} \mathbf{1}$ was accomplished by a different synthetic strategy, involving indolylpiperazines $\mathbf{9 a}-\mathbf{b}$ [35], which were reacted with $N$-chloroacetyl benzoxazines $\mathbf{4 d}-\mathbf{f}$ and $4 \mathbf{j}-\mathbf{1}$ to give the aforementioned six final compounds $(7 \mathbf{d}-\mathbf{f}$ and $\mathbf{7 j}-\mathbf{1})$ with good to excellent yields (Scheme 3). 
<smiles>[R]c1ccc2[nH]cc(CCCN3CCNCC3)c2c1</smiles>

4d-f

$$
R=H\left\{\begin{array}{l}
R_{1}=C l, R_{2}=H, 7 d \\
R_{1}=B r, R_{2}=H, 7 e \\
R_{1}=O C H_{3}, R_{2}=H, 7 f
\end{array} \quad R=F \quad\left\{\begin{array}{l}
R_{1}=C l, R_{2}=H, 7 j \\
R_{1}=B r, R_{2}=H, 7 k \\
R_{1}=O C H_{3}, R_{2}=H, 7 l
\end{array}\right.\right.
$$

Scheme 3. Synthesis of Series I. Route 2. Reagents and conditions: $\mathrm{K}_{2} \mathrm{CO}_{3}, \mathrm{CH}_{3} \mathrm{CN}, 80^{\circ} \mathrm{C}$, Yield (63-91\%).

In summary, 15 compounds were synthesized for Series I in yields ranging from $42 \%$ to $91 \%$.

\subsection{Chemistry Series II}

(2-\{4-[3-(1H-Indol-3-yl)-propyl]-1-piperazinyl\}-acetylamine)-N-(2-morpholin-4-yl-ethyl)-fluorinated benzamides

The synthetic pathway of this series involved the fluorinated benzamide derivatives 12a-d, which were obtained from commercially available isomeric fluoro nitrobenzoic acids in a three-step sequence with good to excellent yields as shown in Scheme 4.

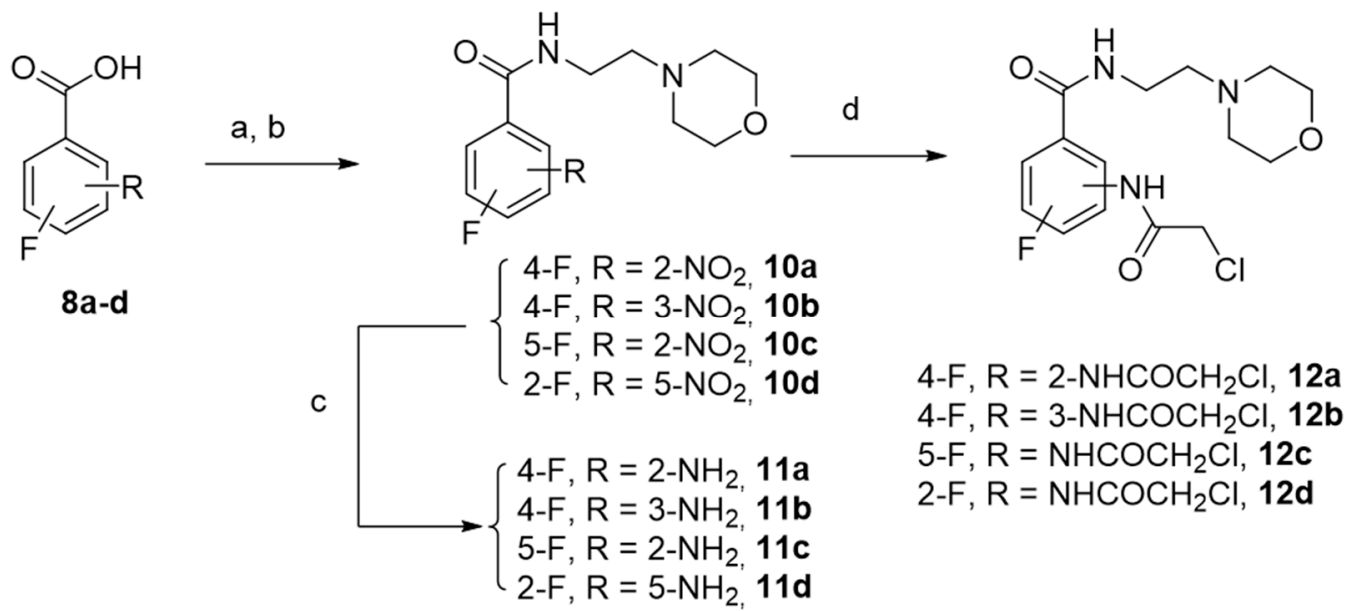

Scheme 4. Synthesis of derivatives 12a-d. Reagents and experimental conditions: (a) $\mathrm{SOCl}_{2}, 60{ }^{\circ} \mathrm{C}$; (b) 2-aminoethyl morpholine, anhydrous THF; (c) $\mathrm{Fe}^{\circ} /\left(\mathrm{H}_{2} \mathrm{O} / \mathrm{EtOH} / \mathrm{CH}_{3} \mathrm{COOH}\right) / 60^{\circ} \mathrm{C}$; (81-98\%) (d) 2-chloroacetylchloride, anhydrous THF, $0{ }^{\circ} \mathrm{C}$, Yield (93-97\%).

The fluorinated benzamides derivatives 12a-d were finally connected to indolylpropylpiperazines 9a-c to achieve the expected compounds 13a-1, with yields ranging from $47 \%$ to $85 \%$ (Scheme 5). 
$\mathrm{R}$

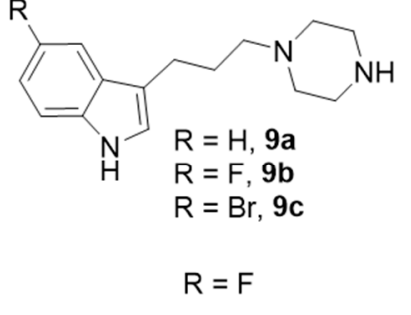

5-F, 2-CONHCH${ }_{2} \mathrm{CH}_{2}$ Morph, 13a 2-F, 5- $\mathrm{CONHCH}_{2} \mathrm{CH}_{2}$ Morph, 13b 4-F, 5- $\mathrm{CONHCH}_{2} \mathrm{CH}_{2}$ Morph, 13c 4-F, 2-CONHCH${ }_{2} \mathrm{CH}_{2}$ Morph, 13d

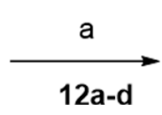

$\mathrm{R}=\mathrm{Br}$

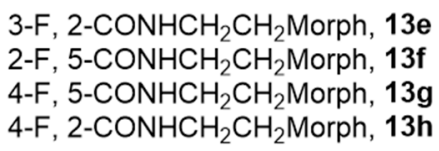

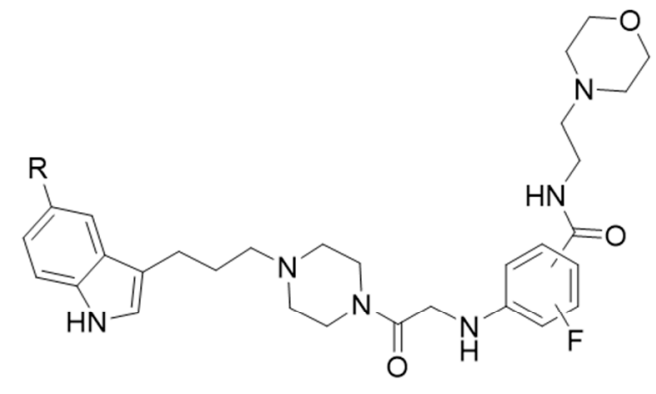

$\mathrm{R}=\mathrm{H}$

5-F, 2-CONHCH $\mathrm{CH}_{2}$ Morph, 13i 2-F, 5- $\mathrm{CONHCH}_{2} \mathrm{CH}_{2}$ Morph, 13j 4-F, 5- $\mathrm{CONHCH} \mathrm{CH}_{2}$ Morph, $13 \mathrm{k}$ 4-F, 2- $\mathrm{CONHCH}_{2} \mathrm{CH}_{2}$ Morph, 13I

Scheme 5. Synthesis of Series II derivatives 13a-1. Reagents and conditions: $\mathrm{K}_{2} \mathrm{CO}_{3}, \mathrm{CH}_{3} \mathrm{CN}, 80{ }^{\circ} \mathrm{C}$, Yield (47-85\%).

\subsection{Pharmacology Series I}

Table 1 summarizes the affinity of compounds $7 \mathbf{a}-\mathbf{o}$ for SERT, $\mathrm{D}_{2}$ receptor, and MAO-A. Most compounds were potent and clearly selective as SERT ligands, showing in all cases affinities in the nanomolar range, whereas the affinities for $\mathrm{D}_{2}$ and MAO-A ranged from micromolar to much higher values, respectively. A detailed analysis of SERT activities indicates that a C-5 substitution of the indole ring with halogens (fluorine or bromine; compounds $7 \mathrm{~g}$ or $7 \mathrm{~m}$ ) leads to more potent compounds than the unsubstituted derivative (7a). On the other hand, the presence of a halogen atom at C-6 of the benzoxazine ring increased the affinity (e.g., 7c, 7d, and 7e vs. 7a). Accordingly, the most potent compounds were those exhibiting a dual halogen substitution pattern $(\mathbf{7 i}, \mathbf{7 j}$, and $\mathbf{7 k}$ ), with $\mathrm{Ki}$ values below $10 \mathrm{nM}$. The C-7 halogen substitution on the benzoxazine ring gave no consistent effects, slight increases $(\mathbf{7 b}$ and $\mathbf{7 h})$ or decreases $(\mathbf{7 n})$ of affinity were observed, as compared with the corresponding C-7 unsubstituted compounds $\left(7 \mathbf{a}, 7 \mathrm{~g}\right.$, and $7 \mathrm{~m}$, respectively). The $\mathrm{D}_{2}$ receptor affinity for this series indicates that no conclusive structure-activity relationships can be extracted for these compounds. Nevertheless, it is apparent that dihalogenated derivatives, bearing one halogen atom at the C- 5 of the indole ring and the other at either the C- 6 or C-7 of the benzoxazine moiety $(\mathbf{7 h}-\mathbf{7 k}, \mathbf{7 m}-\mathbf{7 o})$, resulted in more potent compounds than their corresponding monohalogenated or unsubstituted counterparts $(7 \mathbf{a}-7 \mathbf{e}, 7 \mathbf{g})$. Moreover, the presence of a methoxyl group at the C-6 of the benzoxazine ring has almost no effect on the affinity of the compounds for $\mathrm{D}_{2}$ receptor. It is worth mentioning that the dihalogenated compound $\mathbf{7 n}$ was the only derivative displaying comparable affinities for SERT and $\mathrm{D}_{2}$ receptor $\left(\mathrm{D}_{2} / \mathrm{SERT}\right.$ ratio $\left.=3.6\right)$ and could be considered as a potential leader in the search of more potent multitarget compounds. 
Table 1. Affinities, measured as Ki values at the serotonin transporter (SERT), $\mathrm{D}_{2}$ receptor, and percent of monoamine oxidase-A (MAO-A) inhibition (at $100 \mu \mathrm{M}$ ) of indolepiperazinyl benzoxazine derivatives (Series I).

\begin{tabular}{|c|c|c|c|c|c|c|}
\hline Compound & $\mathbf{R}$ & $\mathbf{R}_{\mathbf{1}}$ & $\mathbf{R}_{2}$ & SERT (nM) & $\mathrm{D}_{2}(\mathrm{nM})$ & $\begin{array}{c}\% \text { Inhibition } \\
\text { MAO-A at } 100 \mu \mathrm{M}\end{array}$ \\
\hline Fluoxetine & - & - & - & $3.15 \pm 0.32$ & N.D. & N.D. \\
\hline Haloperidol & - & - & - & N.D. & $3.88 \pm 0.44$ & N.D. \\
\hline $7 a$ & $-\mathrm{H}$ & $-\mathrm{H}$ & $-\mathrm{H}$ & $307.10 \pm 30.46$ & $4840 \pm 68$ & $27 \%$ \\
\hline $7 \mathrm{~b}$ & $-\mathrm{H}$ & $-\mathrm{H}$ & $-F$ & $180.80 \pm 19.97$ & $4740 \pm 131$ & $43 \%$ \\
\hline $7 \mathrm{c}$ & $-\mathrm{H}$ & $-F$ & $-\mathrm{H}$ & $68.78 \pm 1.02$ & $2387 \pm 163$ & $40 \%$ \\
\hline $7 d$ & $-\mathrm{H}$ & $-\mathrm{Cl}$ & $-\mathrm{H}$ & $81.39 \pm 4.97$ & $1265 \pm 182$ & $18 \%$ \\
\hline $7 e$ & $-\mathrm{H}$ & $-\mathrm{Br}$ & $-\mathrm{H}$ & $36.57 \pm 0.55$ & $910 \pm 263$ & $38 \%$ \\
\hline $7 f$ & $-\mathrm{H}$ & $-\mathrm{OCH}_{3}$ & $-\mathrm{H}$ & $412.20 \pm 57.86$ & $4407 \pm 647$ & $58 \%$ \\
\hline $7 \mathrm{~g}$ & $-F$ & $-\mathrm{H}$ & $-\mathrm{H}$ & $9.63 \pm 0.31$ & $2315 \pm 64$ & $41 \%$ \\
\hline $7 \mathrm{~h}$ & $-F$ & $-\mathrm{H}$ & $-F$ & $7.61 \pm 0.02$ & $2317 \pm 163$ & $0 \%$ \\
\hline $7 \mathbf{i}$ & $-F$ & $-F$ & $-\mathrm{H}$ & $7.51 \pm 0.12$ & $1681 \pm 79$ & $0 \%$ \\
\hline $7 \mathbf{j}$ & $-F$ & $-\mathrm{Cl}$ & $-\mathrm{H}$ & $12.30 \pm 2.31$ & $902 \pm 4$ & $20 \%$ \\
\hline $7 \mathbf{k}$ & $-F$ & $-\mathrm{Br}$ & $-\mathrm{H}$ & $5.63 \pm 0.82$ & $1041 \pm 232$ & $20 \%$ \\
\hline 71 & $-F$ & $-\mathrm{OCH}_{3}$ & $-\mathrm{H}$ & $12.60 \pm 1.52$ & $3322 \pm 256$ & $52 \%$ \\
\hline $7 \mathrm{~m}$ & $-\mathrm{Br}$ & $-\mathrm{H}$ & $-\mathrm{H}$ & $50.18 \pm 2.47$ & $593 \pm 62$ & $30 \%$ \\
\hline $7 n$ & $-\mathrm{Br}$ & $-\mathrm{H}$ & $-F$ & $84.44 \pm 2.03$ & $307 \pm 6$ & $23 \%$ \\
\hline 70 & $-\mathrm{Br}$ & $-F$ & $-\mathrm{H}$ & $34.89 \pm 0,32$ & $1152 \pm 227$ & $26 \%$ \\
\hline
\end{tabular}

Data represent the mean \pm SEM of three experiments, each with triplicate samples. All Ki values are in nM. N.D. not determined.

\subsection{Docking Simulation Series I}

Considering the pharmacological results, docking studies aimed to rationalize the molecular interactions and binding modes of the designed compounds were carried out only in the human SERT (hSERT) and in selected cases at the $\mathrm{D}_{2}$ receptor.

\subsection{1. hSERT}

The most potent compounds $\mathbf{7 g}, \mathbf{7 h}, \mathbf{7} \mathbf{i}$, and $\mathbf{7 k}$ showed a common docking pose (Figure 1 ), which favors the following stabilizing interactions: A $\pi-\pi$ interaction between the indole ring and the $\pi$-donor aromatic residue Tyr176, a coulombic interaction between the protonated piperazine $\mathrm{N}-1$ with the Asp98 residue, and a $\pi$-cation interaction for the protonated piperazine with Tyr95. Furthermore, aromatic interactions were also observed for the benzoxazine ring with the residues Phe341 and Phe335. These drug-target interactions are in agreement with those described in the crystal structure of the hSERT in complex with the inhibitor (S)-citalopram [47,48]. 


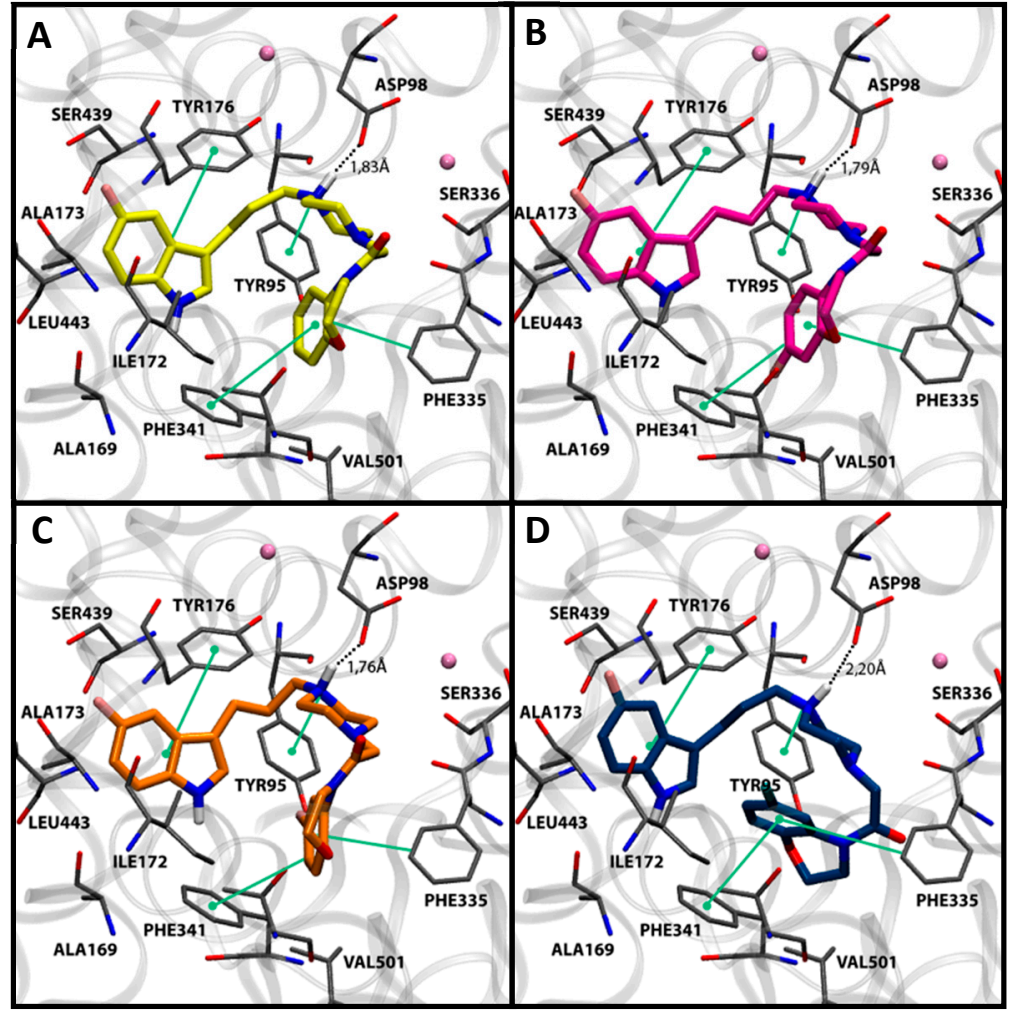

Figure 1. Docking poses in SERT obtained for compounds $7 \mathrm{~g}(\mathrm{~A})$ in yellow, $7 \mathrm{~h}(\mathbf{B})$ in magenta, $7 \mathbf{i}(\mathrm{C})$ in orange, and 7k (D) in blue. Nearby residues $<5 \AA$ (grey sticks) and $\mathrm{Na}^{+}$atoms (pink spheres) are shown. Dotted lines represent ionic interactions and aromatic interactions are shown with green lines.

The relevance of the fluorinated substitution on the indole ring is clearly evidenced by comparison of compounds $\mathbf{7 f}$ and $7 \mathbf{l}$. Both derivatives share the same substitution pattern in the benzoxazine ring, differing only by the presence of a fluorine atom at the indole moiety, which induces a different docking pose for $\mathbf{7 f}$. Thus, the least potent compound of the series (7f) adopted a binding mode in which both indole and piperazine ring interactions are clearly less favored than the C-5 fluorinated counterpart (Figure 2). Compounds showing intermediate affinities (7a-7e and $\mathbf{7 m}-\mathbf{7 o}$ ) exhibited docking poses between the most and least favorable binding modes (not shown).

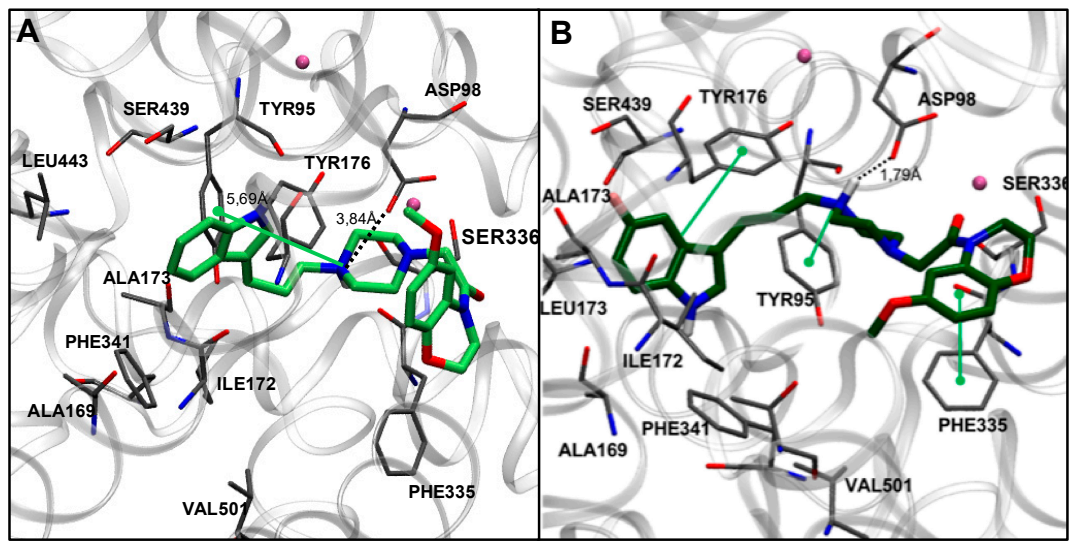

Figure 2. Comparison of the docking poses in SERT obtained for compounds $7 \mathbf{f}(\mathbf{A})$ in light green and 71 (B) in dark green. Nearby residues $<5 \AA$ (grey sticks) and $\mathrm{Na}^{+}$atoms (pink spheres) are shown. Dotted lines represent ionic interactions and aromatic interactions are shown with green lines. 


\subsection{2. $\mathrm{D}_{2}$ Receptor}

Docking simulations showed that compounds of this series adopt, at the $\mathrm{D}_{2}$ receptor, a binding mode similar to that experimentally determined for the atypical antipsychotic risperidone [49]. Thus, the indole moiety appears located into the deep hydrophobic sub-pocket of the orthosteric site, lined by Cys118, Thr119, Ser197, Phe198, and Trp386, while the protonated piperazine N-1 locates in a favorable position to establish a coulombic interaction with Asp114 (Figure 3). Furthermore, the benzoxazine portion extends to the additional hydrophobic sub-pocket lined by Val91, Trp100, Phe110, and Tyr408, in a similar fashion to that observed in the crystal structure for the pyrimidinone moiety of risperidone. Interestingly, this general binding mode was observed for both the most and the least potent compounds of this series $(\mathbf{7 a}, \mathbf{7 b}$, and $\mathbf{7 m}, \mathbf{7 n}$ ), respectively (Figure $3 \mathrm{~A}, \mathrm{~B})$. Therefore, it is tempting to speculate that the higher affinity showed by brominated derivatives $(7 \mathbf{m}$ and $\mathbf{7 n}$ ) is due to the formation of a halogen bond between the bromine and a hydroxyl group of an adjacent residue (e.g., Ser197). As observed (Figure 3), this could also change the position of the benzoxazine moiety, favoring its interactions at the more external hydrophobic sub-pocket.

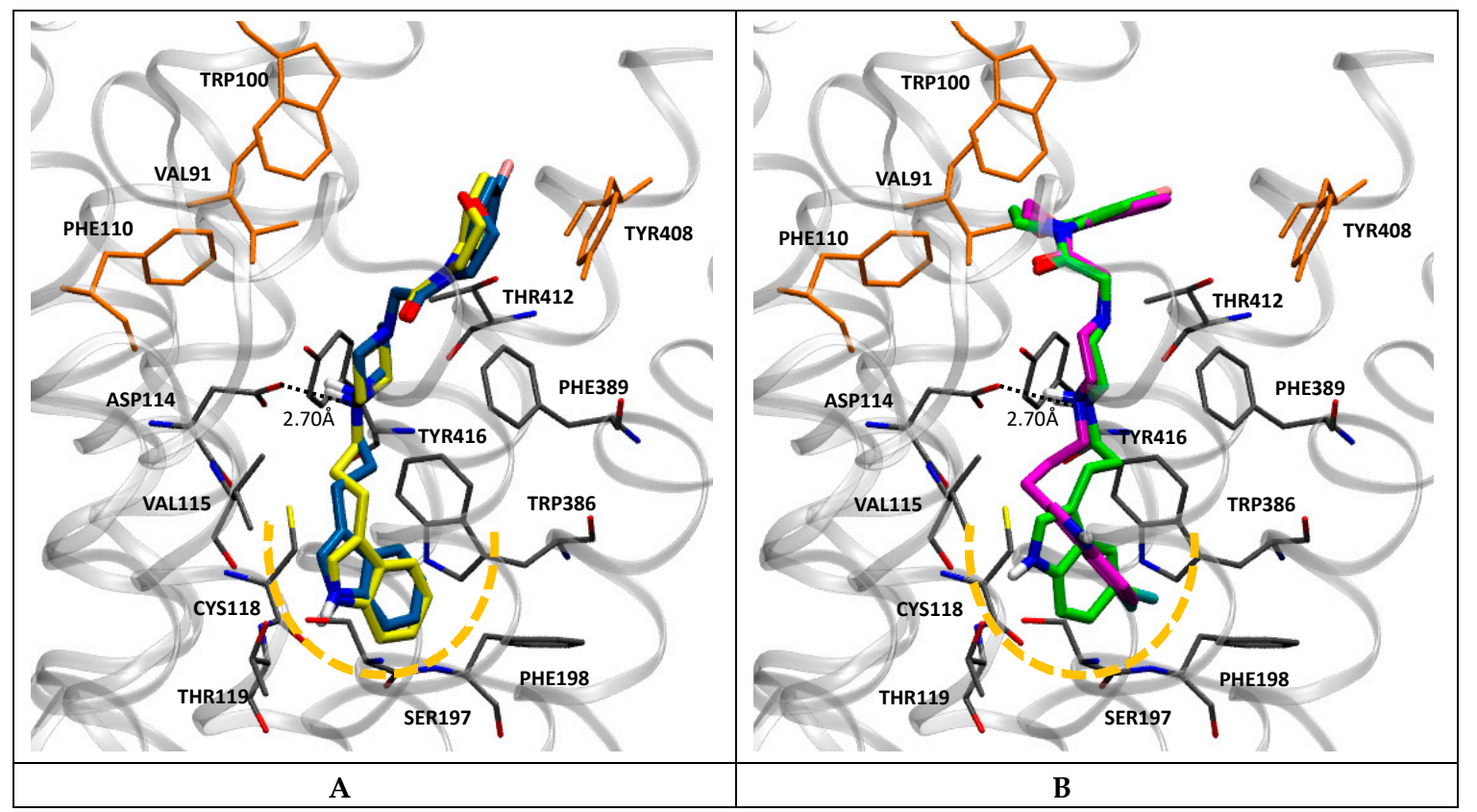

Figure 3. Docking poses in $\mathrm{D}_{2}$ obtained for compounds $7 \mathbf{a}$ in yellow and $7 \mathbf{b}$ in blue (A), and compounds $\mathbf{7 m}$ in magenta and $\mathbf{7 n}$ in green $(\mathbf{B})$. Nearby residues $<5 \AA$ (grey sticks) are shown. Dotted lines represent ionic interactions. Yellow segmented line represents the deep hydrophobic sub-pocket. Residues lining the more external hydrophobic sub-pocket are shown in orange.

\subsection{Pharmacology Series II}

Table 2 summarizes the affinity of Series II compounds 13a-131 for SERT, $\mathrm{D}_{2}$ receptor, and MAO-A. As in the case of indole benzoxazine derivatives (Series I), most indole morpholine ethylbenzamides (Series II) were potent SERT ligands, showing much lower affinities for $\mathrm{D}_{2}$ receptor and virtually no effect upon MAO-A activity. Regarding SERT activity, and in agreement with our previous studies, halogen substitution at $\mathrm{C}-5$ of the indole ring with fluorine or bromine (compounds 13a-g) conducted an increase in affinity as compared with the unsubstituted analogues $\mathbf{1 3} \mathbf{i}-\mathbf{1}$, with the fluoro derivatives 13a-d being the most potent of the series. On the other hand, when the acetanilide portion, connected to the indolylpropylpiperazinyl fragment, was functionalized with a fluorine atom (at C-2) and a morpholino ethylcarboxamide, the best affinities were obtained when the bulkier substituent was located at meta position (compounds 13c, 13g, and 13k). 
Table 2. Affinities, measured as Ki values at SERT, $\mathrm{D}_{2}$ receptor, and percent of MAO-A inhibition (at $100 \mu \mathrm{M}$ ) of indolepiperazinylmorpholinoethyl benzamide derivatives (Series II).

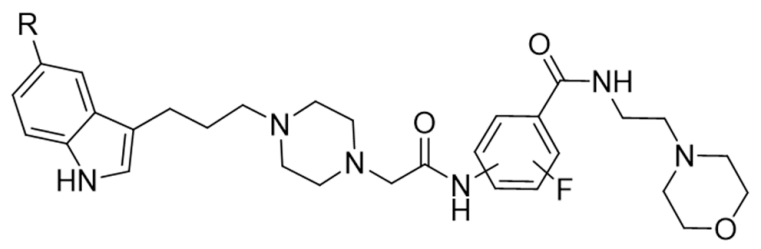

\begin{tabular}{|c|c|c|c|c|c|c|}
\hline Compound & $\mathbf{R}$ & $\begin{array}{c}\text { Position } \\
\text { (Indolylpropyl } \\
\text { Piperazineacetamide) }\end{array}$ & $\begin{array}{l}\text { Position } \\
\quad \text { F }\end{array}$ & SERT (nM) & $\mathrm{D}_{2}(\mathrm{nM})$ & $\begin{array}{c}\% \text { Inhibition } \\
\text { MAO-A at } 100 \mu \mathrm{M}\end{array}$ \\
\hline Fluoxetine & - & - & - & $3.15 \pm 0.32$ & N.D. & N.D. \\
\hline Haloperidol & - & - & - & N.D. & $3.88 \pm 0.44$ & N.D. \\
\hline $13 a$ & $\mathrm{~F}$ & 2 & 4 & $16.04 \pm 0.21$ & $5455 \pm 25$ & $19 \%$ \\
\hline $13 b$ & $\mathrm{~F}$ & 3 & 4 & $23.78 \pm 0.46$ & $3100 \pm 42$ & $42 \%$ \\
\hline $13 c$ & $\mathrm{~F}$ & 5 & 2 & $6.83 \pm 0.19$ & $592.60 \pm 30.22$ & $28 \%$ \\
\hline $13 d$ & $\mathrm{~F}$ & 2 & 5 & $98.14 \pm 1.24$ & $9060 \pm 29$ & $30 \%$ \\
\hline $13 e$ & $\mathrm{Br}$ & 2 & 4 & $222.60 \pm 16.44$ & $5619 \pm 31$ & $39 \%$ \\
\hline $13 \mathrm{f}$ & $\mathrm{Br}$ & 3 & 4 & $58.85 \pm 1.14$ & $1832 \pm 33$ & $39 \%$ \\
\hline $13 \mathrm{~g}$ & $\mathrm{Br}$ & 5 & 2 & $12.62 \pm 0.42$ & $765 \pm 16$ & $16 \%$ \\
\hline $13 \mathrm{~h}$ & $\mathrm{Br}$ & 2 & 5 & $199.60 \pm 14.53$ & $4700 \pm 168$ & $9.4 \%$ \\
\hline $13 \mathbf{i}$ & $\mathrm{H}$ & 2 & 4 & $9945 \pm 383$ & $48910 \pm 1279$ & $23 \%$ \\
\hline $13 j$ & $\mathrm{H}$ & 3 & 4 & $109.90 \pm 3.26$ & $2339 \pm 45$ & $44 \%$ \\
\hline $13 k$ & $\mathrm{H}$ & 5 & 2 & $26.81 \pm 0.91$ & $4169 \pm 335$ & $34 \%$ \\
\hline 131 & $\mathrm{H}$ & 2 & 5 & $52.16 \pm 3.24$ & $2000 \pm 34$ & $34 \%$ \\
\hline
\end{tabular}

Data represent the mean \pm SEM of three experiments, each with triplicate samples. All Ki values are in nM range. N.D. no determined.

\subsection{Docking Simulation Series II}

Similar to the analysis of Series I and considering the pharmacological results, docking studies were carried out only in hSERT. In this series, seven compounds exhibited Ki values between 7 and $60 \mathrm{nM}(13 \mathrm{a}, 13 \mathrm{~b}, 13 \mathrm{c}, 13 \mathrm{f}, 13 \mathrm{~g}, 13 \mathrm{k}$, and 131). Docking simulations showed that compounds with the lowest $\mathrm{Ki}$ values (13c and $\mathbf{1 3 g}$ ) share a common binding mode into the S1 site of the SERT, which is similar to that described for compounds of Series I (Figure 4A). Thus, the piperazine N-1 can establish ionic and $\pi$-cation interactions with Asp98 and Tyr176, respectively, while the indole moiety can participate in aromatic interactions with Tyr176 and Phe341. Interestingly, the ethylmorpholinic chain extends towards the extracellular vestibule (also known as the S2 site). On the other hand, for the compounds with the lowest affinities (13e and 13i), docking simulations showed that the piperazine $\mathrm{N}-1$ was located farther away from Asp98 and Tyr95, making the possible ionic interactions with these residues unlikely or much weaker (Figure 4B). The analysis of the docking poses indicates that the most potent compounds, i.e., those having a 5,2-substitution pattern (13c, 13g, and 13k) exhibited an extended conformation at the binding site, while the least potent compounds (13e and 13i, showing a 2,4-substitution pattern) adopted a more constrained binding mode, impairing the most relevant interactions. 


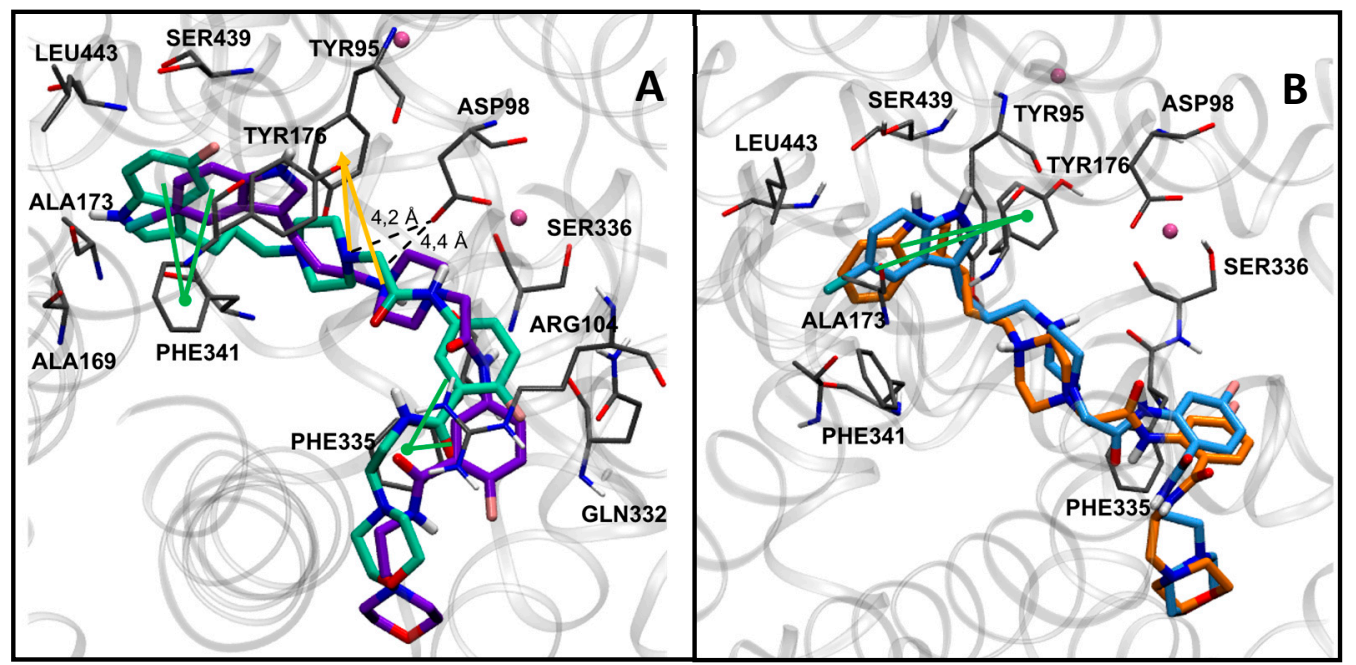

Figure 4. Docking poses in SERT obtained for compounds 13c in cyan and 13g in purple (A), and 13e in light blue, and 13i in orange (B). Nearby residues $<5 \AA$ (grey sticks) and $\mathrm{Na}^{+}$atoms (pink spheres) are shown. Dotted lines represent ionic interactions, orange lines represent $\pi$-cation interactions, and aromatic interactions are shown with green lines.

\subsection{3-D-QSAR Study}

To systematize the structure-activity relationship of the synthesized molecules, we carried out a 3-D-QSAR study of the CoMFA and CoMSIA type. The complete series of 27 molecules was divided into training (19 compounds) and test sets (8 compounds) in a ratio of 70:30, selecting the test set compounds at random to avoid bias. The $\mathrm{q}^{2}$ values for the best models were 0.625 and 0.523 for CoMFA and CoMSIA, respectively while the $\mathrm{r}^{2}{ }_{\text {ncv }}$ values were 0.967 and 0.959 for CoMFA and CoMSIA, respectively. The statistical summary, as well as the tables of affinities for both models and their respective graphs, are incorporated in the Supplementary Material.

The steric contour map of CoMFA (Figure 5A) shows a green polyhedron on the bromine atom of compound $\mathbf{7 k}$, the most active of the series. This means that the insertion of bulky atoms or groups in this position is favorable for biological activity. This is consistent with docking studies showing that compounds of series I place halogen into the void space close to lipophilic residues like Trp100 and Tyr408. In the case of compounds of series II, the meta-substituted benzamides placed the chain towards the green region, not the ortho-substituted ones, so it is preferable that the chains are in the meta-position. This is confirmed in the docking of these compounds, in which better accommodation is observed in the SERT binding site. On the other hand, the electrostatic contour map (Figure 5B) shows three blue polyhedra of significant size. This means that the presence of positively charged atoms in these positions would be favorable for affinity. Such polyhedra are located on the carbon atom bonded to the halogen in the case of series I, suggesting that the presence of electronegative atoms bonded to the aforementioned carbon is favorable. The second blue polyhedron is localized on the oxygen atom of the carbonyl group belonging to the ortho-substituted series II amide-compounds. Therefore, oxygen atom remotion would be favorable for affinity. Finally, the third polyhedron is observed on the oxygen atom of the morpholine ring in the ortho-substituted compounds for series II, indicating that changing the morpholine by a piperazine or piperidine ring should lead to better affinities. Furthermore, alkyl chains substitutions at the ortho-position in the benzamide ring resulted in less favorable affinities compared to meta substitutions as was experimentally corroborated. 


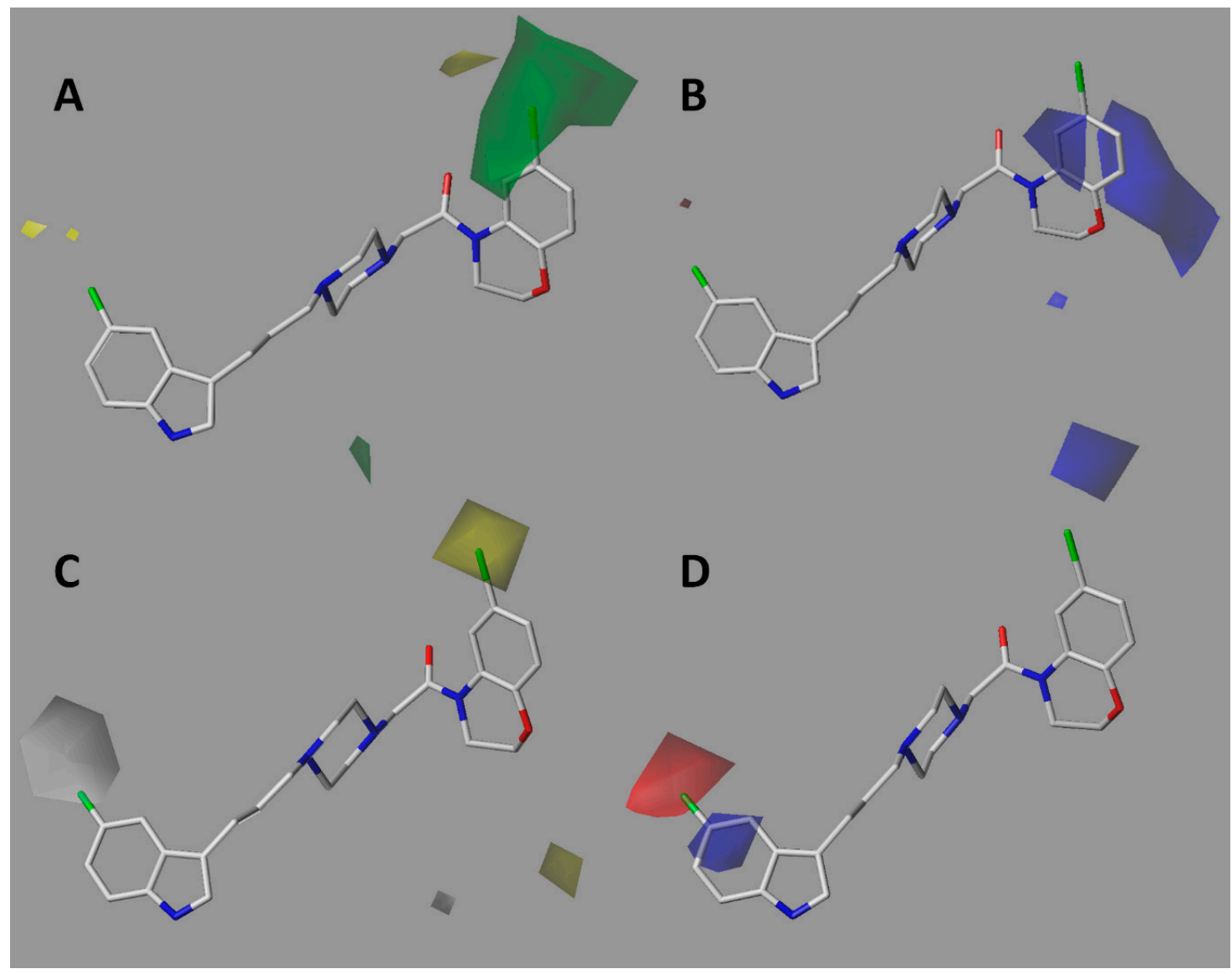

Figure 5. CoMFA $(\mathbf{A}, \mathbf{B})$ and CoMSIA $(\mathbf{C}, \mathbf{D})$ contour maps for the entire series of compounds evaluated in SERT. The compound with the best SERT affinity (7k) is depicted. A. Steric contour map. Green means that large groups are favorable for activity. B and D. Electrostatic contour maps. Red means electron-rich atoms are favorable and blue means positively charged atoms are favorable. C. Hydrophobic map. Gray means that hydrophilic groups are favorable for activity and yellow means that hydrophobic groups are favorable.

The hydrophobic contour map of CoMSIA (Figure 5C) showed a gray polyhedron at position C5 of the indole ring, meaning that the presence of hydrophilic groups is favorable for affinity. In fact, the $\mathrm{C} 5$ fluorine-substituted indoles displayed the best affinities of the series. Other polar groups like $\mathrm{OH}, \mathrm{NH}_{2}$, or $\mathrm{NR}_{2}$ would also be interesting to evaluate at this position. Similarly, a yellow polyhedron located on the bromine atom of the benzoxazine framework (compound $7 \mathbf{k}$ ) means that the presence of lipophilic groups is favorable for activity. In concordance, halogens like $\mathrm{Cl}, \mathrm{Br}$, and I would be the most appropriated substituents and groups, such as aromatic rings, alkyl, and/or alkoxy chains, could also be explored. In the case of compounds of series II, a yellow polyhedron is located on the amide group of the meta-substituted compounds; therefore, the replacement of the amide by a less-polar function, such as a ketone or ester, would be an interesting option to explore. On the other hand, the electrostatic contour map of CoMSIA (Figure 5D) showed two polyhedra around compound 7k. A red polyhedron on the halogen atom at position 5 of the indole ring means that the presence of electron-rich atoms is favorable for affinity. It is interesting to note that the blue polyhedron intersecting the carbon atom of indole at position 5 is complementary to the red polyhedron. In consequence, the presence of a positive charge on the indole ring is favorable for activity. Other potential electron-withdrawing groups to be explored are $\mathrm{CN}, \mathrm{NO}_{2}$, and $\mathrm{COR}$. Docking studies showed $\pi$-stacking interaction between the $\pi$-deficient indole ring with Tyr176, Phe341, and Trp386 residues. 


\section{Materials and Methods}

\subsection{General Methods}

Melting points were determined on a hot-stage apparatus and were uncorrected. The ${ }^{1} \mathrm{H}$ and ${ }^{13} \mathrm{C}-\mathrm{NMR}$ spectra were obtained on a Bruker DRX-300 spectrometer (300 and $75 \mathrm{MHz}$, respectively) in $\mathrm{CDCl}_{3}, \mathrm{DMSO}-d_{6}$, and $\mathrm{CD}_{3} \mathrm{COCD}_{3}-d_{6}$. Chemical shifts were recorded in ppm $(\delta)$ relative to TMS as an internal standard. $J$ values are given in Hz. Micro-analyses were carried out on a Fisons EA 1108 analyzer. High-resolution mass spectra were recorded on a DSA-TOFAxION 2 TOF MS (Perkin Elmer, Shelton, CT, USA), positive mode. Silica gel Merck 60 (70-230 mesh) and aluminum sheets coated with silica gel 60 F254 were used for column and TLC chromatography, respectively.

3.1.1. General Procedure for the Synthesis of [4-[2-(3,4-Dihydro-2H-benzo[b][1,4]oxazin-4-yl)-2-oxoethyl]-piperazine-1-yl] tert-butylcarbamate Derivatives 5a-c

[4-[2-(3,4-Dihydro-2H-benzo[b][1,4]oxazin-4-yl)-2-oxo-ethyl]-1-piperazinyl] tert-butylcarbamate (5a) as a Model

To a solution containing 2-Chloro-1-(2,3-dihydrobenzo[b][1,4]oxazin-4-yl) ethanamide 4a (1.5 g; $7.09 \mathrm{mmol})$ in dry $\mathrm{CH}_{3} \mathrm{CN}(60 \mathrm{~mL}), \mathrm{N}$-Boc-piperazine $(1321 \mathrm{mg} ; 7.09 \mathrm{mmol})$ and anhydrous $\mathrm{K}_{2} \mathrm{CO}_{3}$ (980 mg; $7.09 \mathrm{mmol}$ ) were added. The mixture was stirred at $80^{\circ} \mathrm{C}$ for $24 \mathrm{~h}$. After this time, the mixture was diluted with water $(100 \mathrm{~mL})$ and the solution extracted with EtOAc $(100 \mathrm{~mL} \times 3)$, dried over anhydrous $\mathrm{Na}_{2} \mathrm{SO}_{4}$, and concentrated under reduced pressure. The organic crude was purified by silica gel column chromatography with EtOAc as eluent, to provide 5a (2152 mg; 84\% yield) as a white solid. m.p.: $133.0-134.0{ }^{\circ} \mathrm{C} ;{ }^{1} \mathrm{H}-\mathrm{NMR}\left(\mathrm{CDCl}_{3}\right): \delta 1.45$ (s, 9H, H-4'), 2.52 (t, 4H, H-3' , J = 4.8 Hz), 3.39 (s, $\left.2 \mathrm{H}, \mathrm{H}-1^{\prime}\right), 3.45\left(\mathrm{t}, 4 \mathrm{H}, \mathrm{H}-2^{\prime}, J=4.5 \mathrm{~Hz}\right), 3.99(\mathrm{t}, 2 \mathrm{H}, \mathrm{H}-3, J=4.8 \mathrm{~Hz}), 4.31(\mathrm{t}, 2 \mathrm{H}, \mathrm{H}-2, J=4.4 \mathrm{~Hz}), 6.91(\mathrm{~m}$, 2H, H-6 and H-8), $7.08(\mathrm{t}, 1 \mathrm{H}, \mathrm{H}-7, J=7.3 \mathrm{~Hz})$, and 7.99 (br. s, $1 \mathrm{H}, \mathrm{H}-5)$ ppm. ${ }^{13} \mathrm{C}-\mathrm{NMR}\left(\mathrm{CDCl}_{3}\right): \delta 27.9$ (3X), 42.6, 43.2 (2X), $52.4(2 X), 55.4,66.4,79.3,116.8,119.7,123.5,125.6,146.3,154.2,167.3$, and 167.5 ppm. HRMS: (EI) Calculated for $\mathrm{C}_{19} \mathrm{H}_{27} \mathrm{~N}_{3} \mathrm{O}_{4}\left(\mathrm{M}^{+}\right)=362.20799$. Found: 362.2113.

[4-[2-(7-Fluoro-2,3-dihydro-benzo[b][1,4]oxazin-4-yl)-2-oxo-ethyl]-1-piperazinyl] tert-butylcarbamate $(\mathbf{5 b})$

2-Chloro-1-(7-fluoro-2,3-dihidro-benzo [1,4]oxazin-4-yl)-ethanamide 4b (1.5 g; $6.53 \mathrm{mmol})$, $\mathrm{N}$-Boc-piperazine (1216 mg; $6.53 \mathrm{mmol}$ ), and anhydrous $\mathrm{K}_{2} \mathrm{CO}_{3}(902 \mathrm{mg} ; 6.53 \mathrm{mmol}$ ), to afford $5 \mathbf{b}$ (1983 mg; 80\% yield) as a white solid. m.p.: $126.6-128.5^{\circ} \mathrm{C} ;{ }^{1} \mathrm{H}-\mathrm{NMR}\left(\mathrm{CDCl}_{3}\right): \delta 1.46\left(\mathrm{~s}, 9 \mathrm{H}, \mathrm{H}-4^{\prime}\right)$, $2.51\left(\mathrm{t}, 4 \mathrm{H}, \mathrm{H}-2^{\prime}, J=4.9 \mathrm{~Hz}\right), 3.35\left(\mathrm{~s}, 2 \mathrm{H}, \mathrm{H}-1^{\prime}\right), 3.45\left(\mathrm{t}, 4 \mathrm{H}, \mathrm{H}-3^{\prime}, J=4.8 \mathrm{~Hz}\right), 3.97(\mathrm{t}, 2 \mathrm{H}, \mathrm{H}-3, J=4.8$ $\mathrm{Hz}), 4.30(\mathrm{t}, 2 \mathrm{H}, \mathrm{H}-2, \mathrm{~J}=4.1 \mathrm{~Hz}), 6.58-6.66(\mathrm{~m}, 2 \mathrm{H}, \mathrm{H}-6$, and H-8), and $8.03(\mathrm{br} . \mathrm{s}, 1 \mathrm{H}, \mathrm{H}-5) \mathrm{ppm}$. ${ }^{13} \mathrm{C}-\mathrm{NMR}\left(\mathrm{CDCl}_{3}\right): \delta 27.9(3 \mathrm{X}), 42.543 .2(2 \mathrm{X}), 52.4(2 \mathrm{X}), 62.1,66.4,79.3,103.8\left(\mathrm{~d},{ }^{2} \mathrm{~J}_{\mathrm{C}-\mathrm{F}}=27 \mathrm{~Hz}\right), 106.7(\mathrm{~d}$, $\left.{ }^{2} J_{\mathrm{C}-\mathrm{F}}=22.5 \mathrm{~Hz}\right), 121.8\left(\mathrm{~d},{ }^{3} J_{\mathrm{C}-\mathrm{F}}=9.3 \mathrm{~Hz}\right), 124.6\left(\mathrm{~d},{ }^{4} J_{\mathrm{C}-\mathrm{F}}=8.2 \mathrm{~Hz}\right), 147.3\left(\mathrm{~d},{ }^{3^{\prime}} J_{\mathrm{C}-\mathrm{F}}=6 \mathrm{~Hz}\right), 154.2,155.6$ $\left(\mathrm{d},{ }^{1} J_{\mathrm{C}-\mathrm{F}}=222 \mathrm{~Hz}\right)$, and 167 ppm. HRMS: (EI) Calculated for $\mathrm{C}_{19} \mathrm{H}_{26} \mathrm{FN}_{3} \mathrm{O}_{4}\left(\mathrm{M}^{+}\right)=380.19856$. Found: 380.2047 .

[4-[2-(6-Fluoro-2,3-dihydro-benzo[b][1,4]oxazin-4-yl)-2-oxo-ethyl]-1-piperazinyl] tert-butylcarbamate $(5 \mathrm{c})$

2-Chloro-1-(6-fluoro-2,3-dihidro-benzo [1,4]oxazin-4-yl)-ethanamide 4c (1.5 g; $6.53 \mathrm{mmol})$, $\mathrm{N}$-Boc-piperazine (1216 mg; $6.53 \mathrm{mmol}$ ), and anhydrous $\mathrm{K}_{2} \mathrm{CO}_{3}(902 \mathrm{mg} ; 6.53 \mathrm{mmol}$ ), to afford 5c (2033 mg; 82\% yield) as a white solid. m.p.: 113.4-114.5 ${ }^{\circ} \mathrm{C} ;{ }^{1} \mathrm{H}-\mathrm{NMR}\left(\mathrm{CDCl}_{3}\right): \delta 1.46\left(\mathrm{~s}, 9 \mathrm{H}, \mathrm{H}-4^{\prime}\right)$, $2.53\left(\mathrm{t}, 4 \mathrm{H}, \mathrm{H}-2^{\prime}, \mathrm{J}=4.8 \mathrm{~Hz}\right), 3.35\left(\mathrm{~s}, 2 \mathrm{H}, \mathrm{H}-1^{\prime}\right), 3.46\left(\mathrm{t}, 4 \mathrm{H}, \mathrm{H}-3^{\prime}, J=4.8 \mathrm{~Hz}\right), 3.98(\mathrm{t}, 2 \mathrm{H}, \mathrm{H}-3, J=4.9 \mathrm{~Hz})$, $4.27(\mathrm{t}, 2 \mathrm{H}, \mathrm{H}-2, \mathrm{~J}=4.4 \mathrm{~Hz}), 6.77-6.87\left(\mathrm{~m}, 2 \mathrm{H}, \mathrm{H}-6\right.$ and H-8), and 7.82 (br. s, 1H, H-5) ppm. ${ }^{13} \mathrm{C}-\mathrm{NMR}$ $\left(\mathrm{CDCl}_{3}\right): \delta 27.9(3 \mathrm{X}), 42.543 .4(2 \mathrm{X}), 52.3(2 \mathrm{X}), 62.1,65.6,79.3,110.1\left(\mathrm{~d},{ }^{2} J_{\mathrm{C}-\mathrm{F}}=28.5 \mathrm{~Hz}\right), 112.2(\mathrm{~d}$, $\left.{ }^{2} J_{\mathrm{C}-\mathrm{F}}=23.6 \mathrm{~Hz}\right), 117.1\left(\mathrm{~d},{ }^{3} J_{\mathrm{C}-\mathrm{F}}=9.3 \mathrm{~Hz}\right), 125.7\left(\mathrm{~d},{ }^{4} J_{\mathrm{C}-\mathrm{F}}=4.9 \mathrm{~Hz}\right), 142.2\left(\mathrm{~d},{ }^{3} J_{\mathrm{C}-\mathrm{F}}=9.3 \mathrm{~Hz}\right), 154.2,155.5$ $\left(\mathrm{d},{ }^{1} J_{\mathrm{C}-\mathrm{F}}=238 \mathrm{~Hz}\right)$, and $167.1 \mathrm{ppm}$. HRMS: (EI) Calculated for $\mathrm{C}_{19} \mathrm{H}_{26} \mathrm{FN}_{3} \mathrm{O}_{4}\left(\mathrm{M}^{+}\right)=380.19856$. Found: 380.2043. 
3.1.2. General Procedure for the Synthesis of 1-(2,3-dihydro-benzo[1,4]oxazin-4-yl)-2-piperazin-1-ylethanamide Derivatives 6a-c

Synthesis of 1-(2,3-Dihydro-benzo[b][1,4] oxazin-4-yl)-2-(1-piperazinyl) ethanamide (6a) as a Model

A mixture of [4-[2-(3,4-Dihydro-2H-benzo[b][1,4]oxazin-4-yl)-2-oxo-ethyl]-1-piperazinyl] tert-butylcarbamate $5 \mathrm{a}(2 \mathrm{~g} ; 5.53 \mathrm{mmol})$ in dry $\mathrm{CH}_{2} \mathrm{Cl}_{2}(20 \mathrm{~mL})$ and trifluoroacetic acid $(12 \mathrm{~mL})$ was stirred at $0{ }^{\circ} \mathrm{C}$, for $4 \mathrm{~h}$. After this time, dry $\mathrm{CH}_{2} \mathrm{Cl}_{2}(200 \mathrm{~mL})$ was added and neutralized with solid $\mathrm{NaHCO}_{3}(10 \mathrm{~g})$ to later filter on celite. The mixture was finally diluted with a saturated solution of $\mathrm{NaHCO}_{3}(200 \mathrm{~mL})$, extracted with EtOAc $(8 \times 50 \mathrm{~mL})$, dried over anhydrous $\mathrm{Na}_{2} \mathrm{SO}_{4}$, and concentrated under vacuum to obtain pure $6 \mathbf{a}(867 \mathrm{mg} ; 82 \%$ yield) as an unstable yellow light solid, highly hygroscopic; ${ }^{1} \mathrm{H}-\mathrm{NMR}$ (DMSO- $\left.\mathrm{d}_{6}\right): \delta 2.37\left(\mathrm{~m}, 4 \mathrm{H}, \mathrm{H}-2^{\prime}\right), 2.69\left(\mathrm{~m}, 4 \mathrm{H}, \mathrm{H}-3^{\prime}\right), 3.29(\mathrm{~s}, 2 \mathrm{H}$, $\left.\mathrm{H}-1^{\prime}\right), 3.89(\mathrm{t}, 2 \mathrm{H}, \mathrm{H}-3, J=4.4 \mathrm{~Hz}), 4.25(\mathrm{t}, 2 \mathrm{H}, \mathrm{H}-2, J=4.1 \mathrm{~Hz}), 6.85(\mathrm{~m}, 2 \mathrm{H}, \mathrm{H}-6$ and H-8), $7.0(\mathrm{t}, 1 \mathrm{H}$, $\mathrm{H}-7, J=7.6 \mathrm{~Hz}$ ) and 7.85 (br. s, $1 \mathrm{H}, \mathrm{H}-5$ ) ppm. ${ }^{13} \mathrm{C}-\mathrm{NMR}$ (DMSO- $d_{6}$ ): $\delta 40.5,45.2(2 \mathrm{X}), 53.2(2 \mathrm{X}), 62.0$, $66.1,73.5,116.7,119.6,123.8,125.1,146.2$, and $167.8 \mathrm{ppm}$.

1-(7-Fluoro-2,3-dihydro-benzo[b][1,4]oxazin-4-yl)-2-(1-piperazinyl) ethanamide (6b)

[4-[2-(7-Fluoro-2,3-dihydro-benzo[b][1,4] oxazin-4-yl)-2-oxo-ethyl]-1-piperazinyl]tertbutyl carbamate $5 \mathbf{b}(2 \mathrm{~g} ; 5.27 \mathrm{mmol})$ and trifluoroacetic acid $(12 \mathrm{~mL})$, in dry $\mathrm{CH}_{2} \mathrm{Cl}_{2}(20 \mathrm{~mL})$ reacted to provide $\mathbf{6 b}$ (1398 mg; 95\% yield) as a brown light hygroscopic compound; ${ }^{1} \mathrm{H}-\mathrm{NMR}\left(\mathrm{CDCl}_{3}\right): \delta 2.57$ $\left(\mathrm{m}, 4 \mathrm{H}, \mathrm{H}-2^{\prime}\right), 2.94\left(\mathrm{t}, 4 \mathrm{H}, \mathrm{H}-3^{\prime}, J=4.4 \mathrm{~Hz}\right), 3.18$ (br. s, $\left.1 \mathrm{H}, \mathrm{H}-4^{\prime}\right), 3.33\left(\mathrm{~s}, 2 \mathrm{H}, \mathrm{H}-1^{\prime}\right), 3.97(\mathrm{t}, 2 \mathrm{H}, \mathrm{H}-3$, $J=4.8 \mathrm{~Hz}), 4.31(\mathrm{t}, 2 \mathrm{H}, \mathrm{H}-2, J=4.2 \mathrm{~Hz}), 6.59-6.65(\mathrm{~m}, 2 \mathrm{H}, \mathrm{H}-6$, and $\mathrm{H}-8)$, and $8.0(\mathrm{br} . \mathrm{s}, 1 \mathrm{H}, \mathrm{H}-5)$ ppm. ${ }^{13} \mathrm{C}-\mathrm{NMR}\left(\mathrm{CDCl}_{3}\right): \delta 45.6(2 \mathrm{X}), 53.8(2 \mathrm{X}), 60.4,66.9,77.3,104.2\left(\mathrm{~d},{ }^{2} J_{\mathrm{C}-\mathrm{F}}=24 \mathrm{~Hz}\right), 107.2(\mathrm{~d}$, $\left.{ }^{2} J_{\mathrm{C}-\mathrm{F}}=23 \mathrm{~Hz}\right), 114.5\left(\mathrm{~d},{ }^{4} J_{\mathrm{C}-\mathrm{F}}=7 \mathrm{~Hz}\right), 125.1\left(\mathrm{~d},{ }^{3} J_{\mathrm{C}-\mathrm{F}}=9.4 \mathrm{~Hz}\right), 143.4\left(\mathrm{~d},{ }^{3} J_{\mathrm{C}-\mathrm{F}}=15 \mathrm{~Hz}\right), 155.7\left(\mathrm{~d},{ }^{1} J_{\mathrm{C}-\mathrm{F}}\right.$ $=250 \mathrm{~Hz})$, and $171.2 \mathrm{ppm}$.

1-(6-Fluoro-2,3-dihydro-benzo[b][1,4]oxazin-4-yl)-2-(1-piperazinyl) ethanamide (6c)

[4-[2-(6-Fluoro-2,3-dihydro-benzo[b][1,4] oxazin-4-yl)-2-oxo-ethyl]-1-piperazinyl]tertbutyl carbamate $5 \mathrm{c}(2 \mathrm{~g} ; 5.27 \mathrm{mmol})$ and trifluoroacetic acid $(12 \mathrm{~mL})$, in dry $\mathrm{CH}_{2} \mathrm{Cl}_{2}(20 \mathrm{~mL})$ reacted to provide $6 \mathrm{c}\left(1369 \mathrm{mg} ; 93 \%\right.$ yield) as a brown light hygroscopic compound; ${ }^{1} \mathrm{H}-\mathrm{NMR}\left(\mathrm{CDCl}_{3}\right): \delta 2.62(\mathrm{~m}$, $\left.4 \mathrm{H}, \mathrm{H}-2^{\prime}\right), 2.99\left(\mathrm{t}, 4 \mathrm{H}, \mathrm{H}-3^{\prime}, \mathrm{J}=4.6 \mathrm{~Hz}\right), 3.35\left(\mathrm{~s}, 2 \mathrm{H}, \mathrm{H}-\mathrm{1}^{\prime}\right), 3.97(\mathrm{~m}, 3 \mathrm{H}, \mathrm{H}-3$ and H-4' $), 4.28(\mathrm{t}, 2 \mathrm{H}, \mathrm{H}-2$, $J=4.5 \mathrm{~Hz}), 6.76-6.87(\mathrm{~m}, 2 \mathrm{H}, \mathrm{H}-7$ and $\mathrm{H}-8)$ and 7.71 (br. s, $1 \mathrm{H}, \mathrm{H}-5) \mathrm{ppm} ;{ }^{13} \mathrm{C}-\mathrm{NMR}\left(\mathrm{CDCl}_{3}\right): \delta 45.6$ (2X), $53.8(2 \mathrm{X}), 60.4,66.9,77.2,110.6\left(\mathrm{~d},{ }^{2} J_{\mathrm{C}-\mathrm{F}}=28 \mathrm{~Hz}\right), 112.8\left(\mathrm{~d},{ }^{2} J_{\mathrm{C}-\mathrm{F}}=32 \mathrm{~Hz}\right), 117.6\left(\mathrm{~d},{ }^{3} J_{\mathrm{C}-\mathrm{F}}=9.4 \mathrm{~Hz}\right)$, $126.1\left(\mathrm{~d},{ }^{3^{\prime}} J_{\mathrm{C}-\mathrm{F}}=11 \mathrm{~Hz}\right), 142.8\left(\mathrm{~d},{ }^{4} J_{\mathrm{C}-\mathrm{F}}=6.6 \mathrm{~Hz}\right), 157.6\left(\mathrm{~d},{ }^{1} J_{\mathrm{C}-\mathrm{F}}=237 \mathrm{~Hz}\right)$, and $171.2 \mathrm{ppm}$.

3.1.3. General Procedure for the Synthesis of 2,3-dihydro-benzo[b][1,4] oxazin-4-yl)-2-\{4-[3-(1H-3indolyl)-propyl]-1-piperazinyl\} ethanamide Derivatives $7 \mathbf{a}-\mathbf{c}, 7 \mathbf{g}-\mathbf{i}$, and $\mathbf{7 m - o}$. Method 1

1-(2,3-Dihydro-benzo[b][1,4]oxazin-4-yl)-2-\{4-[3-(1H-3-indolyl)-propyl]-1-piperazinyl\} ethanamide (7a) as a Model

To a stirred solution of 3-(1H-3-indolyl)-propyl-4-methylbencensulfonate 1a (254 mg. $0.77 \mathrm{mmol}$ ), in dry $\mathrm{CH}_{3} \mathrm{CN}(50 \mathrm{~mL})$, benzoxazine $6 \mathbf{a}(200 \mathrm{mg}, 0.77 \mathrm{mmol})$ and anhydrous $\mathrm{K}_{2} \mathrm{CO}_{3}(106 \mathrm{mg} ; 0.77 \mathrm{mmol})$ were added. The mixture was heated at $80^{\circ} \mathrm{C}$ for $24 \mathrm{~h}$. After this time, the resulting mixture was poured into water $(100 \mathrm{~mL})$, extracted with EtOAc $(4 \times 50 \mathrm{~mL})$, dried over anhydrous $\mathrm{Na}_{2} \mathrm{SO}_{4}$, and concentrated under reduced pressure. The organic crude was purified by column chromatography EtOAc/MeOH (6:1) to give compound $7 \mathrm{a}\left(217 \mathrm{mg} ; 70 \%\right.$ yield) as a yellow light solid; m.p.: $124.3-125.8{ }^{\circ} \mathrm{C} ;{ }^{1} \mathrm{H}-\mathrm{NMR}$ (DMSO- $\left.d_{6}\right): \delta 1.77\left(\mathrm{q}, 2 \mathrm{H}, \mathrm{H}-2^{\prime}, J=7.0 \mathrm{~Hz}\right), 2.22-2.50\left(\mathrm{~m}, 10 \mathrm{H}, \mathrm{H}-3^{\prime}, \mathrm{H}-4^{\prime}\right.$ and $\left.\mathrm{H}-5^{\prime}\right), 2.67\left(\mathrm{t}, 2 \mathrm{H}, \mathrm{H}-1^{\prime}\right.$, $J=7.4 \mathrm{~Hz}), 3.33\left(\mathrm{~s}, 2 \mathrm{H}, \mathrm{H}-7^{\prime}\right), 3.90\left(\mathrm{t}, 2 \mathrm{H}, \mathrm{H}-3^{\prime \prime}, J=3.8 \mathrm{~Hz}\right), 4.26\left(\mathrm{t}, 2 \mathrm{H}, \mathrm{H}-2^{\prime \prime}, J=3.7 \mathrm{~Hz}\right), 6.85(\mathrm{~m}, 2 \mathrm{H}$, $\mathrm{H}-6^{\prime \prime}$ and H-8" $), 6.95$ (t, 1H, H-7" $\left.J=7.7 \mathrm{~Hz}\right), 7.03(\mathrm{~m}, 2 \mathrm{H}, \mathrm{H}-5$ and $\mathrm{H}-6), 7.09(\mathrm{~d}, 1 \mathrm{H}, \mathrm{H}-2, J=1.4 \mathrm{~Hz})$, 7.33 (d, 1H, H-4, J = 7.8 Hz), 7.49 (d, 1H, H-7, J = $7.7 \mathrm{~Hz}$ ), 7.90 (br. s, $1 \mathrm{H}, \mathrm{H}-5^{\prime \prime}$ ), and 10.79 (br. s, $1 \mathrm{H}$, H-1) ppm. ${ }^{13}$ C-NMR (DMSO- $d_{6}$ ): $\delta 22.9,27.6,39.2,40.8,53.0$ (2X), 53.2 (2X), 58.0, 66.6, 111.8, 114.8, 
117.2, 118.5, 118.7, 120.1, 121.2, 122.6, 124.3, 126.0, 127.7, 128.5, 136.7, 146.7, and 168.3 ppm. HRMS: (EI) Calculated for $\mathrm{C}_{25} \mathrm{H}_{30} \mathrm{~N}_{4} \mathrm{O}_{2}\left(\mathrm{M}^{+}\right)=419.5313$. Found: 419.4426 .

1-(7-Fluoro-2,3-dihydro-benzo[b][1,4]oxazin-4-yl)-2-\{4-[3-(1H-3-indolyl)-propyl]-1-piperazinyl\} ethanamide $(7 \mathbf{b})$

To a solution of 3-(1H-3-indolyl)-propyl-4-methylbencensulfonate $1 \mathrm{a}$ (237 $\mathrm{mg}$; $0.72 \mathrm{mmol})$, in dry $\mathrm{CH}_{3} \mathrm{CN}(50 \mathrm{~mL})$, 7-fluoro-benzoxazine $6 \mathbf{b}(200 \mathrm{mg} ; 0.72 \mathrm{mmol})$ and anhydrous $\mathrm{K}_{2} \mathrm{CO}_{3}(100 \mathrm{mg}$; $0.72 \mathrm{mmol}$ ) were added. The chromatographic purification provided $7 \mathbf{b}(242 \mathrm{mg} ; 80 \%$ yield) as a yellow light solid. m.p.: $124.0-125.3^{\circ} \mathrm{C} ;{ }^{1} \mathrm{H}-\mathrm{NMR}$ (DMSO- $\left.d_{6}\right): \delta 1.76\left(\mathrm{q}, 2 \mathrm{H}, \mathrm{H}-2^{\prime}, J=7.3 \mathrm{~Hz}\right), 2.22-2.48$

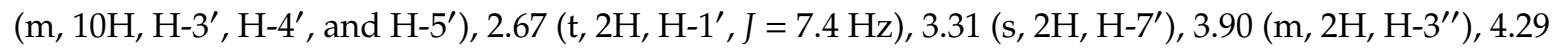
$\left(\mathrm{m}, 2 \mathrm{H}, \mathrm{H}-2^{\prime \prime}\right), 6.68-6.78\left(\mathrm{~m}, 2 \mathrm{H}, \mathrm{H}-6^{\prime \prime}\right.$ and H-8 $\left.{ }^{\prime \prime}\right), 6.95(\mathrm{t}, 1 \mathrm{H}, \mathrm{H}-5$ or H-6, J = 7.1 Hz), $7.04(\mathrm{t}, 1 \mathrm{H}, \mathrm{H}-6$ or $\mathrm{H}-5, J=7.0 \mathrm{~Hz}), 7.09(\mathrm{~d}, 1 \mathrm{H}, \mathrm{H}-2, J=1.8 \mathrm{~Hz}), 7.32(\mathrm{~d}, 1 \mathrm{H}, \mathrm{H}-4, J=8.0 \mathrm{~Hz}), 7.49(\mathrm{~d}, 1 \mathrm{H}, \mathrm{H}-7, J=7.7 \mathrm{~Hz})$, 7.92 (br. s, $1 \mathrm{H}, \mathrm{H}-5^{\prime \prime}$ ), and 10.83 (br. s, $1 \mathrm{H}, \mathrm{H}-1$ ) ppm. ${ }^{13} \mathrm{C}-\mathrm{NMR}$ (DMSO- $d_{6}$ ): $\delta 22.9,27.6,53.0(2 \mathrm{X}), 53.3$ $(2 X), 58.1,67,104.0\left(\mathrm{~d},{ }^{2} J_{\mathrm{C}-\mathrm{F}}=26 \mathrm{~Hz}\right), 106.9\left(\mathrm{~d},{ }^{2} J_{\mathrm{C}-\mathrm{F}}=23 \mathrm{~Hz}\right), 111.8,114.8,118.6\left(\mathrm{~d},{ }^{3} J_{\mathrm{C}-\mathrm{F}}=18 \mathrm{~Hz}\right)$, $121.2,122.6,123.2,125.6\left(\mathrm{~d},{ }^{4} J_{\mathrm{C}-\mathrm{F}}=9 \mathrm{~Hz}\right), 127.7,136.7,143.2,144,147.6,147.8\left(\mathrm{~d},{ }^{3} J_{\mathrm{C}-\mathrm{F}}=13 \mathrm{~Hz}\right), 159.5$ $\left(\mathrm{d},{ }^{1} J_{\mathrm{C}-\mathrm{F}}=240 \mathrm{~Hz}\right)$, and $168.2 \mathrm{ppm}$. HRMS: (EI) Calculated for $\mathrm{C}_{25} \mathrm{H}_{29} \mathrm{FN}_{4} \mathrm{O}_{2}\left(\mathrm{M}^{+}\right)=437.5318$. Found: 437.5301.

1-(6-Fluoro-2,3-dihydro-benzo[b][1,4]oxazin-4-yl)-2-\{4-[3-(1H-3-indolyl)-propyl]-1-piperazinyl\} ethanamide (7c)

To a solution of 3-(1H-3-indolyl)-propyl-4-methylbencensulfonate $1 \mathrm{a}$ (237 mg; $0.72 \mathrm{mmol})$, in dry $\mathrm{CH}_{3} \mathrm{CN}$ (50 mL), 6-fluoro-benzoxazine $6 \mathrm{c}(200 \mathrm{mg} ; 0.72 \mathrm{mmol})$ and anhydrous $\mathrm{K}_{2} \mathrm{CO}_{3}(100 \mathrm{mg}$; $0.72 \mathrm{mmol}$ ) were added. The crude mixture was column chromatographed to provide pure $7 \mathrm{c}(269$ mg; $89 \%$ yield) as a yellow light solid. m.p.: $123.8-125.1{ }^{\circ} \mathrm{C} ;{ }^{1} \mathrm{H}-\mathrm{NMR}$ (DMSO- $\left.d_{6}\right): \delta 1.83\left(\mathrm{q}, 2 \mathrm{H}, \mathrm{H}-2^{\prime}\right.$, $J=7.1 \mathrm{~Hz}), 2.30-2.54\left(\mathrm{~m}, 10 \mathrm{H}, \mathrm{H}-3^{\prime}, \mathrm{H}-4^{\prime}\right.$, and H-5'), $2.73\left(\mathrm{t}, 2 \mathrm{H}, \mathrm{H}-1^{\prime}, J=7.4 \mathrm{~Hz}\right), 3.40\left(\mathrm{~s}, 2 \mathrm{H}, \mathrm{H}-7^{\prime}\right)$, $3.98\left(\mathrm{t}, 2 \mathrm{H}, \mathrm{H}-3^{\prime \prime}, J=4.0 \mathrm{~Hz}\right), 4.30\left(\mathrm{t}, 2 \mathrm{H}, \mathrm{H}-2^{\prime \prime}, J=3.7 \mathrm{~Hz}\right), 6.91-6.97\left(\mathrm{~m}, 2 \mathrm{H}, \mathrm{H}-\mathrm{7}^{\prime \prime}\right.$ and H-8" $), 7.01$ (td, $1 \mathrm{H}, \mathrm{H}-5$ or H-6, $\left.J_{o}=7.3 \mathrm{~Hz}, J_{m}=1.0 \mathrm{~Hz}\right), 7.11\left(\mathrm{td}, 1 \mathrm{H}, \mathrm{H}-6\right.$ or H-5, $\left.J_{o}=7.0 \mathrm{~Hz}, J_{m}=1.1 \mathrm{~Hz}\right), 7.15(\mathrm{~d}, 1 \mathrm{H}$, $\mathrm{H}-2, J=2.1 \mathrm{~Hz}), 7.38(\mathrm{~d}, 1 \mathrm{H}, \mathrm{H}-4, J=8.0 \mathrm{~Hz}$ ), 7.55 (d, 1H, H-7, J = 7.7 Hz), 7.95 (br. d, 1H, H-5",$J=11.7$ $\mathrm{Hz}$ ), and 10.81 (br. s, $1 \mathrm{H}, \mathrm{H}-1$ ) ppm. ${ }^{13} \mathrm{C}-\mathrm{NMR}$ (DMSO- $d_{6}$ ): $\delta$ 22.9, 27.6, $53.0(2 \mathrm{X}), 53.3(2 \mathrm{X}), 58.1,66.1$, $110.3\left(\mathrm{~d},{ }^{2} J_{\mathrm{C}-\mathrm{F}}=29 \mathrm{~Hz}\right), 111.9\left(\mathrm{~d},{ }^{\prime} J_{\mathrm{C}-\mathrm{F}}=25 \mathrm{~Hz}\right), 114.8,117.9\left(\mathrm{~d},{ }^{3} J_{\mathrm{C}-\mathrm{F}}=9 \mathrm{~Hz}\right), 118.5,118.7,121.2,122.6$, $126.9\left(\mathrm{~d},{ }^{3} J_{\mathrm{C}-\mathrm{F}}=12 \mathrm{~Hz}\right), 127.7,128.7,129.9,136.7,138.3,143\left(\mathrm{~d},{ }^{4} J_{\mathrm{C}-\mathrm{F}}=2 \mathrm{~Hz}\right), 155.5\left(\mathrm{~d},{ }^{1} J_{\mathrm{C}-\mathrm{F}}=239 \mathrm{~Hz}\right)$, and 168.7 ppm. HRMS: (EI) Calculated for $\mathrm{C}_{25} \mathrm{H}_{29} \mathrm{FN}_{4} \mathrm{O}_{2}\left(\mathrm{M}^{+}\right)=437.5318$. Found: 437.5084.

1-(2,3-Dihydro-benzo[b][1,4] oxazin-4-yl)-2-\{4-[3-(5-fluoro-1H-3-indolyl)-propyl]-1-piperazinyl\} ethanamide $(7 \mathrm{~g})$

To a solution of 3-(5-Fluoro-1H-3-indolyl)-propyl-4-methylbencensulfonate $\mathbf{1 b}$ ( $268 \mathrm{mg}$; $0.77 \mathrm{mmol})$ in $\mathrm{CH}_{3} \mathrm{CN}$ (50 mL), 1-(2,3-Dihydro-benzo[b][1,4] oxazin-4-yl)-2-(1-piperazinyl) ethanamide $6 \mathbf{6}$ (200 mg; $0.77 \mathrm{mmol}$ ) and anhydrous $\mathrm{K}_{2} \mathrm{CO}_{3}(106 \mathrm{mg} ; 0.77 \mathrm{mmol})$ were added. The crude mixture was column chromatographed to provide pure $7 \mathrm{~g}\left(252 \mathrm{mg} ; 78 \%\right.$ yield) as a yellow light solid. m.p.: $168.4-171.3^{\circ} \mathrm{C}$; ${ }^{1} \mathrm{H}-\mathrm{NMR}$ (DMSO- $\left.d_{6}\right): \delta 1.76\left(\mathrm{q}, 2 \mathrm{H}, \mathrm{H}-2^{\prime}, J=6.9 \mathrm{~Hz}\right), 2.30-2.51\left(\mathrm{~m}, 10 \mathrm{H}, \mathrm{H}-3^{\prime}, \mathrm{H}-4^{\prime}\right.$ and $\left.\mathrm{H}-5^{\prime}\right), 2.63$ $\left(\mathrm{t}, 2 \mathrm{H}, \mathrm{H}-\mathrm{1}^{\prime}, J=7.3 \mathrm{~Hz}\right), 3.34\left(\mathrm{~s}, 2 \mathrm{H}, \mathrm{H}-7^{\prime}\right), 3.89\left(\mathrm{~m}, 2 \mathrm{H}, \mathrm{H}-3^{\prime \prime}\right), 4.26\left(\mathrm{t}, 2 \mathrm{H}, \mathrm{H}-2^{\prime \prime}, J=3.6 \mathrm{~Hz}\right), 6.75(\mathrm{td}, 1 \mathrm{H}$, $\left.\mathrm{H}-6, J_{o}=10.4 \mathrm{~Hz}, J_{m}=2.7 \mathrm{~Hz}\right), 6.80-6.91\left(\mathrm{~m}, 2 \mathrm{H}, \mathrm{H}-6^{\prime \prime}\right.$, and H-8" $), 7.01\left(\mathrm{t}, 1 \mathrm{H}, \mathrm{H}-7^{\prime \prime}, J=7.4 \mathrm{~Hz}\right), 7.18$ $(\mathrm{d}, 1 \mathrm{H}, \mathrm{H}-2, J=1.8 \mathrm{~Hz}), 7.23\left(\mathrm{dd}, 1 \mathrm{H}, \mathrm{H}-7, J_{o}=10.2 \mathrm{~Hz}, J_{m}=2.3 \mathrm{~Hz}\right), 7.30\left(\mathrm{dd}, 1 \mathrm{H}, \mathrm{H}-4, J_{o}=8.9 \mathrm{~Hz}\right.$, $J_{m}=4.5 \mathrm{~Hz}$ ), 7.88 (br. s, $\left.1 \mathrm{H}, \mathrm{H}-5^{\prime \prime}\right)$, and $10.89(\mathrm{~s}, 1 \mathrm{H}, \mathrm{H}-1)$ ppm. ${ }^{13} \mathrm{C}-\mathrm{NMR}$ (DMSO- $\left.d_{6}\right): \delta 22.6,27.2,52.7$ $(2 \mathrm{X}), 53.1(2 \mathrm{X}), 57.7,66.1,103.4\left(\mathrm{~d},{ }^{2} J_{\mathrm{C}-\mathrm{F}}=23 \mathrm{~Hz}\right), 104.2,109.3\left(\mathrm{~d},{ }^{2} J_{\mathrm{C}-\mathrm{F}}=26 \mathrm{~Hz}\right), 112.6\left(\mathrm{~d},{ }^{3} J_{\mathrm{C}-\mathrm{F}}=9.9\right.$ $\mathrm{Hz}), 115.1\left(\mathrm{~d},{ }^{4} J_{\mathrm{C}-\mathrm{F}}=4.9 \mathrm{~Hz}\right), 117.2,120.1,124.3,124.9,125.7,126.6,127.9\left(\mathrm{~d},{ }^{\prime} J_{\mathrm{C}-\mathrm{F}}=9.9 \mathrm{~Hz}\right), 133.4$, 146.7, 147.9, $157.3\left(\mathrm{~d},{ }^{1} J_{\mathrm{C}-\mathrm{F}}=234 \mathrm{~Hz}\right.$ ), and $168.2 \mathrm{ppm}$. HRMS: (EI) Calculated for $\mathrm{C}_{25} \mathrm{H}_{29} \mathrm{FN}_{4} \mathrm{O}_{2}\left(\mathrm{M}^{+}\right)=$ 437.5318. Found: 437.5312. 
1-(7-Fluoro-2,3-dihydro-benzo[b][1,4]oxazin-4-yl)-2-\{4-[3-(5-fluoro-1H-3-indolyl)-propyl]-1-piperazinyl\} ethanamide $(7 \mathrm{~h})$

To a solution of 3-(5-Fluoro-1H-3-indolyl)-propyl-4-methylbencensulfonate $\mathbf{1 b}$ ( $268 \mathrm{mg}$; $0.72 \mathrm{mmol}$ ) in $\mathrm{CH}_{3} \mathrm{CN}$ (50 mL), 1-(7-Fluoro-2,3-dihydro-benzo[b][1,4] oxazin-4-yl)-2-(1-piperazinyl) ethanamide 6b (200 mg; $0.72 \mathrm{mmol})$ and anhydrous $\mathrm{K}_{2} \mathrm{CO}_{3}(100 \mathrm{mg} ; 0.72 \mathrm{mmol})$ were added. The crude mixture was column chromatographed to provide pure $7 \mathrm{~h}(205 \mathrm{mg} ; 65 \%$ yield $)$ as a yellow light solid. m.p.: 168.1-169.9 ${ }^{\circ} \mathrm{C} ;{ }^{1} \mathrm{H}-\mathrm{NMR}$ (DMSO- $\left.d_{6}\right): \delta 1.75\left(\mathrm{q}, 2 \mathrm{H}, \mathrm{H}-2^{\prime}, J=7.0 \mathrm{~Hz}\right), 2.25-2.52\left(\mathrm{~m}, 10 \mathrm{H}, \mathrm{H}-3^{\prime}, \mathrm{H}-4^{\prime}\right.$ and H-5'), $2.64\left(\mathrm{t}, 2 \mathrm{H}, \mathrm{H}-1^{\prime}, J=7.4 \mathrm{~Hz}\right), 3.35\left(\mathrm{~s}, 2 \mathrm{H}, \mathrm{H}-7^{\prime}\right), 3.92\left(\mathrm{t}, 2 \mathrm{H}, \mathrm{H}-3^{\prime \prime}, J=3.8 \mathrm{~Hz}\right), 4.25\left(\mathrm{t}, 2 \mathrm{H}, \mathrm{H}-2^{\prime \prime}\right.$, $J=3.7 \mathrm{~Hz}), 6.85-6.93\left(\mathrm{~m}, 3 \mathrm{H}, \mathrm{H}-6, \mathrm{H}-6^{\prime \prime}\right.$, and H-8" $), 7.19(\mathrm{~d}, 1 \mathrm{H}, \mathrm{H}-2, J=2.1 \mathrm{~Hz}), 7.24(\mathrm{dd}, 1 \mathrm{H}, \mathrm{H}-7$, $\left.J_{o}=10.2 \mathrm{~Hz}, J_{m}=2.3 \mathrm{~Hz}\right), 7.31\left(\mathrm{dd}, 1 \mathrm{H}, \mathrm{H}-4, J_{o}=8.8 \mathrm{~Hz}, J_{m}=4.5 \mathrm{~Hz}\right), 7.92\left(\right.$ br. d, $\left.1 \mathrm{H}, \mathrm{H}-5^{\prime \prime}, J=9.6 \mathrm{~Hz}\right)$, and $10.88(\mathrm{~s}, 1 \mathrm{H}, \mathrm{H}-1)$ ppm. ${ }^{13} \mathrm{C}-\mathrm{NMR}$ (DMSO- $\left.d_{6}\right): \delta 22.7,27.5,52.9(2 \mathrm{X}), 53.2(2 \mathrm{X}), 57.8,66.1,103.4(\mathrm{~d}$, $\left.{ }^{2} J_{\mathrm{C}-\mathrm{F}}=23 \mathrm{~Hz}\right), 109.3\left(\mathrm{~d},{ }^{2} J_{\mathrm{C}-\mathrm{F}}=26 \mathrm{~Hz}\right), 110.3\left(\mathrm{~d},{ }^{2 \prime \prime} J_{\mathrm{C}-\mathrm{F}}=28 \mathrm{~Hz}\right), 112.0\left(\mathrm{~d},{ }^{2 \prime \prime \prime} J_{\mathrm{C}-\mathrm{F}}=25 \mathrm{~Hz}\right), 112.6(\mathrm{~d}$, $\left.{ }^{3} J_{\mathrm{C}-\mathrm{F}}=9.9 \mathrm{~Hz}\right), 115.1\left(\mathrm{~d},{ }^{4} J_{\mathrm{C}-\mathrm{F}}=4.9 \mathrm{~Hz}\right), 117.9\left(\mathrm{~d},{ }^{3} J_{\mathrm{C}-\mathrm{F}}=9 \mathrm{~Hz}\right), 124.9,126,126.9\left(\mathrm{~d},{ }^{3 \prime \prime} J_{\mathrm{C}-\mathrm{F}}=9 \mathrm{~Hz}\right), 127.9$ $\left(\mathrm{d},{ }^{3^{\prime \prime \prime}} J_{\mathrm{C}-\mathrm{F}}=9.9 \mathrm{~Hz}\right), 128.5,133.3,143.0\left(\mathrm{~d},{ }^{4^{\prime}} J_{\mathrm{C}-\mathrm{F}}=2.3 \mathrm{~Hz}\right), 155.5\left(\mathrm{~d},{ }^{1} J_{\mathrm{C}-\mathrm{F}}=234 \mathrm{~Hz}\right), 157.0\left(\mathrm{~d},{ }^{{ }^{\prime}} J_{\mathrm{C}-\mathrm{F}}=\right.$ $231 \mathrm{~Hz}$ ), and $168.7 \mathrm{ppm}$. HRMS: (EI) Calculated for $\mathrm{C}_{25} \mathrm{H}_{28} \mathrm{~F}_{2} \mathrm{~N}_{4} \mathrm{O}_{2}\left(\mathrm{M}^{+}\right)=455.5222$. Found: 455.5198 .

1-(6-Fluoro-2,3-dihydro-benzo[b][1,4]oxazin-4-yl)-2-\{4-[3-(5-fluoro-1H-3-indolyl)-propyl]-1-piperazinyl\} ethanamide (7i)

To a solution of 3-(5-Fluoro-1H-3-indolyl)-propyl-4-methylbencensulfonate $\mathbf{1 b}$ ( $268 \mathrm{mg}$; $0.72 \mathrm{mmol}$ ) in $\mathrm{CH}_{3} \mathrm{CN}$ (50 mL), 1-(6-Fluoro-2,3-dihydro-benzo[b][1,4] oxazin-4-yl)-2-(1-piperazinyl) ethanamide 6c (200 mg; $0.72 \mathrm{mmol})$ and anhydrous $\mathrm{K}_{2} \mathrm{CO}_{3}(100 \mathrm{mg} ; 0.72 \mathrm{mmol})$ were added to afford pure $7 \mathbf{i}$ (196 $\mathrm{mg} ; 62 \%$ yield) as a yellow light solid. m.p.: $166.8-168.1{ }^{\circ} \mathrm{C} ;{ }^{1} \mathrm{H}-\mathrm{NMR}$ (DMSO- $d_{6}$ ): $\delta 1.75$ (q, $2 \mathrm{H}, \mathrm{H}-2^{\prime}$, $J=7.0 \mathrm{~Hz}), 2.22-2.55\left(\mathrm{~m}, 10 \mathrm{H}, \mathrm{H}-3^{\prime}, \mathrm{H}-4^{\prime}\right.$ and $\left.\mathrm{H}-5^{\prime}\right), 2.64\left(\mathrm{t}, 2 \mathrm{H}, \mathrm{H}-1^{\prime}, J=7.4 \mathrm{~Hz}\right), 3.35\left(\mathrm{~s}, 2 \mathrm{H}, \mathrm{H}-7^{\prime}\right), 3.92$ $\left(\mathrm{t}, 2 \mathrm{H}, \mathrm{H}-3^{\prime \prime}, J=3.8 \mathrm{~Hz}\right), 4.25\left(\mathrm{t}, 2 \mathrm{H}, \mathrm{H}-2^{\prime \prime}, J=3.7 \mathrm{~Hz}\right), 6.83-6.94\left(\mathrm{~m}, 3 \mathrm{H}, \mathrm{H}-6, \mathrm{H}-7^{\prime \prime}\right.$ and $\left.\mathrm{H}-8^{\prime \prime}\right), 7.19$ $(\mathrm{d}, 1 \mathrm{H}, \mathrm{H}-2, J=2.1 \mathrm{~Hz}), 7.24\left(\mathrm{dd}, 1 \mathrm{H}, \mathrm{H}-7, J_{o}=10.2 \mathrm{~Hz}, J_{m}=2.3 \mathrm{~Hz}\right), 7.31\left(\mathrm{dd}, 1 \mathrm{H}, \mathrm{H}-4, J_{o}=8.8 \mathrm{~Hz}\right.$, $\left.J_{m}=4.5 \mathrm{~Hz}\right), 7.92\left(\mathrm{br} . \mathrm{d}, 1 \mathrm{H}, \mathrm{H}-5^{\prime \prime}, J=9.6 \mathrm{~Hz}\right)$, and $10.88(\mathrm{~s}, 1 \mathrm{H}, \mathrm{H}-1) \mathrm{ppm} .{ }^{13} \mathrm{C}-\mathrm{NMR}$ (DMSO- $\left.d_{6}\right)$ : $\delta 22.7,27.5,52.9(2 X), 53.2(2 X), 57.8,66.1,103.4\left(\mathrm{~d},{ }^{2} J_{\mathrm{C}-\mathrm{F}}=23 \mathrm{~Hz}\right), 109.3\left(\mathrm{~d},{ }^{2} J_{\mathrm{C}-\mathrm{F}}=26 \mathrm{~Hz}\right), 110.3(\mathrm{~d}$, $\left.{ }^{2 \prime \prime} J_{\mathrm{C}-\mathrm{F}}=28 \mathrm{~Hz}\right), 112.0\left(\mathrm{~d},{ }^{2 \prime \prime \prime} J_{\mathrm{C}-\mathrm{F}}=24.7 \mathrm{~Hz}\right), 112.6\left(\mathrm{~d},{ }^{3} J_{\mathrm{C}-\mathrm{F}}=9.9 \mathrm{~Hz}\right), 115.1\left(\mathrm{~d},{ }^{4} J_{\mathrm{C}-\mathrm{F}}=4.9 \mathrm{~Hz}\right), 117.9$ $\left(\mathrm{d},{ }^{3 \prime \prime} J_{\mathrm{C}-\mathrm{F}}=8.8 \mathrm{~Hz}\right), 124.9,126.9\left(\mathrm{~d},{ }^{3} J_{\mathrm{C}-\mathrm{F}}=13 \mathrm{~Hz}\right), 126,127.9\left(\mathrm{~d}, 3^{\prime \prime \prime \prime} J_{\mathrm{C}-\mathrm{F}}=9.9 \mathrm{~Hz}\right), 129,133.3,143.0$ $\left(\mathrm{d},{ }^{4} J_{\mathrm{C}-\mathrm{F}}=2.2 \mathrm{~Hz}\right), 155.5\left(\mathrm{~d},{ }^{1} J_{\mathrm{C}-\mathrm{F}}=234 \mathrm{~Hz}\right), 157.0\left(\mathrm{~d},{ }^{1} J_{\mathrm{C}-\mathrm{F}}=231 \mathrm{~Hz}\right)$, and $168.6 \mathrm{ppm}$. HRMS: (EI) Calculated for $\mathrm{C}_{25} \mathrm{H}_{28} \mathrm{~F}_{2} \mathrm{~N}_{4} \mathrm{O}_{2}\left(\mathrm{M}^{+}\right)=455.5222$. Found: 455.5205 .

1-(2,3-Dihydro-benzo[b][1,4] oxazin-4-yl)-2-\{4-[3-(5-bromo-1H-3-indolyl)-propyl]-1-piperazinyl\} ethanamide $(7 \mathrm{~m})$

To a solution of 3-(5-Bromo-1H-3-indolyl)-propyl-4-methylbencensulfonate 1c (314 mg; $0.77 \mathrm{mmol}$ ) in $\mathrm{CH}_{3} \mathrm{CN}$ (50 mL), 1-(2,3-Dihydro-benzo[b][1,4] oxazin-4-yl)-2-(1-piperazinyl) ethanamide 6a (200 mg; $0.77 \mathrm{mmol})$ and anhydrous $\mathrm{K}_{2} \mathrm{CO}_{3}(106 \mathrm{mg} ; 0.77 \mathrm{mmol})$ were added. The crude mixture was column chromatographed to provide pure $7 \mathrm{~m}\left(157 \mathrm{mg} ; 42 \%\right.$ yield) as a yellow light solid. m.p.: $90.5-92.4{ }^{\circ} \mathrm{C}$; ${ }^{1} \mathrm{H}-\mathrm{NMR}$ (DMSO- $\left.d_{6}\right): \delta 1.74\left(\mathrm{q}, 2 \mathrm{H}, \mathrm{H}-2^{\prime}, J=7.1 \mathrm{~Hz}\right), 2.25-2.51\left(\mathrm{~m}, 10 \mathrm{H}, \mathrm{H}-3^{\prime}, \mathrm{H}-4^{\prime}\right.$, and H-5'), $2.65(\mathrm{t}$, $\left.2 \mathrm{H}, \mathrm{H}-1^{\prime}, J=7.1 \mathrm{~Hz}\right), 3.34\left(\mathrm{~s}, 2 \mathrm{H}, \mathrm{H}-7^{\prime}\right), 3.90$ (t, 2H, H-3",$\left.J=7.1 \mathrm{~Hz}\right), 4.27\left(\mathrm{t}, 2 \mathrm{H}, \mathrm{H}-2^{\prime \prime}, J=7.1 \mathrm{~Hz}\right)$, 6.81-6.90 (m, 2H, H-6" , and H-8" ), $7.02\left(\mathrm{t}, 1 \mathrm{H}, \mathrm{H}-7^{\prime \prime}, J=7.6 \mathrm{~Hz}\right), 7.10-7.20(\mathrm{~m}, 2 \mathrm{H}, \mathrm{H}-2$, and H-6), 7.30 (d, $1 \mathrm{H}, \mathrm{H}-7, J=8.5 \mathrm{~Hz}), 7.68$ (d, 1H, H-4, J = 1.6 Hz), 7.85 (br. s, 1H, H-5"), and 11.04 (s, 1H, H-1) ppm. ${ }^{13}$ C-NMR (DMSO- $d_{6}$ ): $\delta 22.4,27.6,52.9(2 X), 53.2(2 X), 57.6,66.6,111.2,113.9,114.7,117.2,120.1,121.1$, 123.6, 124.3, 124.5, 125.7, 126, 126.6, 128.5, 129.6, 135.3, 146.7, and 168.2 ppm. HRMS: (EI) Calculated for $\mathrm{C}_{25} \mathrm{H}_{29} \mathrm{BrN}_{4} \mathrm{O}_{2}\left(\mathrm{M}^{+}\right)=498.4374$. Found: 498.4362 .

1-(7-Fluoro-2,3-dihydro-benzo[b][1,4]oxazin-4-yl)-2-\{4-[3-(5-bromo-1H-3-indolyl)-propyl]-1-piperazinyl\} ethanamide (7n)

To a solution of 3-(5-Bromo-1H-3-indolyl)-propyl-4-methylbencensulfonate 1c ( $294 \mathrm{mg}$; $0.72 \mathrm{mmol})$ in $\mathrm{CH}_{3} \mathrm{CN}$ (50 mL), 1-(7-Fluoro-2,3-dihydro-benzo[b][1,4]oxazin-4-yl)-2-(1-piperazinyl) ethanamide 
6b (200 mg; $0.72 \mathrm{mmol})$ and anhydrous $\mathrm{K}_{2} \mathrm{CO}_{3}(100 \mathrm{mg} ; 0.72 \mathrm{mmol})$ were added. The crude mixture was column chromatographed to provide pure $7 \mathbf{n}(180 \mathrm{mg} ; 50 \%$ yield) as a yellow light solid. m.p.: 96.1-98.1 ${ }^{\circ} \mathrm{C} ;{ }^{1} \mathrm{H}-\mathrm{NMR}$ (DMSO- $\left.d_{6}\right): \delta 1.73\left(\mathrm{q}, 2 \mathrm{H}, \mathrm{H}-2^{\prime}, J=6.6 \mathrm{~Hz}\right), 2.18-2.51\left(\mathrm{~m}, 10 \mathrm{H}, \mathrm{H}-3^{\prime}, \mathrm{H}-4^{\prime}\right.$ and $\left.\mathrm{H}-5^{\prime}\right), 2.64\left(\mathrm{t}, 2 \mathrm{H}, \mathrm{H}-1^{\prime}, J=7.0 \mathrm{~Hz}\right), 3.32\left(\mathrm{~s}, 2 \mathrm{H}, \mathrm{H}-7^{\prime}\right), 3.90$ (m, 2H, H-3"'), 4.29 (m, 2H, H-2" ) , 6.65-6.81 (m, 2H, H-6" and H-8" ), 7.10-7.20 (m, 2H, H-2, and H-6), 7.30 (d, 1H, H-7, J = 8.5 Hz), 7.68 (d, 1H, H-4, $J=1.0 \mathrm{~Hz}$ ), 7.95 (br. s, $1 \mathrm{H}, \mathrm{H}-5^{\prime \prime}$ ), and 11.05 (s, $1 \mathrm{H}, \mathrm{H}-1$ ) ppm. ${ }^{13} \mathrm{C}-\mathrm{NMR}$ (DMSO- $d_{6}$ ): $\delta 22.4,27.6,52.9$ (2X), $53.2(2 X), 57.6,66.6,110.3\left(\mathrm{~d},{ }^{2} J_{\mathrm{C}-\mathrm{F}}=28.5 \mathrm{~Hz}\right), 111.2,112.0\left(\mathrm{~d},{ }^{2} J_{\mathrm{C}-\mathrm{F}}=25 \mathrm{~Hz}\right), 113.8,114.7,117.9$ $\left(\mathrm{d},{ }^{3} J_{\mathrm{C}-\mathrm{F}}=8.8 \mathrm{~Hz}\right), 121.1,123.6,124.4,126.9\left(\mathrm{~d},{ }^{3} J_{\mathrm{C}-\mathrm{F}}=12 \mathrm{~Hz}\right), 129.6,135.3,143.0\left(\mathrm{~d},{ }^{4} J_{\mathrm{C}-\mathrm{F}}=2.3 \mathrm{~Hz}\right)$, $155.5\left(\mathrm{~d},{ }^{1} J_{\mathrm{C}-\mathrm{F}}=233 \mathrm{~Hz}\right)$, and $168.7 \mathrm{ppm}$. HRMS: (EI) Calculated for $\mathrm{C}_{25} \mathrm{H}_{28} \mathrm{BrFN}_{4} \mathrm{O}_{2}\left(\mathrm{M}^{+}\right)=516.4278$. Found: 516.4267.

1-(6-Fluoro-2,3-dihydro-benzo[b][1,4]oxazin-4-yl)-2-\{4-[3-(5-bromo-1H-3-indolyl)-propyl]-1-piperazinyl\} ethanamide (7o)

To a solution of 3-(5-Bromo-1H-3-indolyl)-propyl-4-methylbencensulfonate 1c (294 mg; $0.72 \mathrm{mmol}$ ) in $\mathrm{CH}_{3} \mathrm{CN}$ (50 mL), 1-(6-Fluoro-2,3-dihydro-benzo[b][1,4] oxazin-4-yl)-2-(1-piperazinyl) ethanamide 6c $(200 \mathrm{mg} ; 0.72 \mathrm{mmol})$ and anhydrous $\mathrm{K}_{2} \mathrm{CO}_{3}(100 \mathrm{mg} ; 0.72 \mathrm{mmol})$ were added. The crude mixture was column chromatographed to provide pure $7 \mathbf{0}(251 \mathrm{mg} ; 70 \%$ yield) as a yellow light solid. m.p.: 97.2-98.8 ${ }^{\circ} \mathrm{C} ;{ }^{1} \mathrm{H}-\mathrm{NMR}\left(\mathrm{DMSO}-d_{6}\right): \delta 1.74\left(\mathrm{q}, 2 \mathrm{H}, \mathrm{H}-2^{\prime}, J=6.7 \mathrm{~Hz}\right), 2.21-2.52\left(\mathrm{~m}, 10 \mathrm{H}, \mathrm{H}-3^{\prime}, \mathrm{H}-4^{\prime}\right.$ and $\left.\mathrm{H}-5^{\prime}\right), 2.65\left(\mathrm{t}, 2 \mathrm{H}, \mathrm{H}-1^{\prime}, J=7.0 \mathrm{~Hz}\right), 3.35\left(\mathrm{~s}, 2 \mathrm{H}, \mathrm{H}-7^{\prime}\right), 3.92\left(\mathrm{~m}, 2 \mathrm{H}, \mathrm{H}-3^{\prime \prime}\right), 4.24\left(\mathrm{~m}, 2 \mathrm{H}, \mathrm{H}-2^{\prime \prime}\right), 6.84-6.93$ $\left(\mathrm{m}, 2 \mathrm{H}, \mathrm{H}-7^{\prime \prime}\right.$ and $\left.\mathrm{H} 8^{\prime \prime}\right), 7.10-7.21(\mathrm{~m}, 2 \mathrm{H}, \mathrm{H}-2$ and H-6), $7.30(\mathrm{~d}, 1 \mathrm{H}, \mathrm{H}-7, J=8.6 \mathrm{~Hz}), 7.68(\mathrm{~s}, 1 \mathrm{H}, \mathrm{H}-4)$, 7.85 (br. d, $1 \mathrm{H}, \mathrm{H}-5^{\prime \prime}, J=7.8 \mathrm{~Hz}$ ), and 11.04 (s, $1 \mathrm{H}, \mathrm{H}-1$ ) ppm. ${ }^{13} \mathrm{C}-\mathrm{NMR}$ (DMSO- $d_{6}$ ): $\delta 22.4,27.6$, $52.9(2 \mathrm{X}), 53.2(2 \mathrm{X}), 57.7,66.1,110.3\left(\mathrm{~d},{ }^{2} J_{\mathrm{C}-\mathrm{F}}=28.5 \mathrm{~Hz}\right), 111.2,112.0\left(\mathrm{~d},{ }^{2} J_{\mathrm{C}-\mathrm{F}}=22.5 \mathrm{~Hz}\right), 113.8,114.7$, $117.9\left(\mathrm{~d},{ }^{3} J_{\mathrm{C}-\mathrm{F}}=8.8 \mathrm{~Hz}\right), 121.1,123.6,124.4,126,126.9\left(\mathrm{~d},{ }^{3} J_{\mathrm{C}-\mathrm{F}}=12.1 \mathrm{~Hz}\right), 128.5,129.6,132.2,135.3$, 138.2, $143.0\left(\mathrm{~d},{ }^{4} J_{\mathrm{C}-\mathrm{F}}=2.2 \mathrm{~Hz}\right), 155.5\left(\mathrm{~d},{ }^{1} J_{\mathrm{C}-\mathrm{F}}=233 \mathrm{~Hz}\right)$, and $168.7 \mathrm{ppm}$. HRMS: (EI) Calculated for $\mathrm{C}_{25} \mathrm{H}_{28} \mathrm{BrFN}_{4} \mathrm{O}_{2}\left(\mathrm{M}^{+}\right)=516.4278$. Found: 516.4272 .

3.1.4. General procedure for the synthesis of 2,3-Dihydro-benzo[1,4]oxazin-4-yl)-2-\{4-[3-(1H-3indolyl)-propyl]-1-piperazinyl\} ethanamides $\mathbf{7} \mathbf{d}-\mathbf{f}$ and $\mathbf{7 j}-\mathbf{1}$. Method 2

Synthesis of 1-(6-Chloro-2,3-dihydro-benzo[b][1,4]oxazin-4-yl)-2-\{4-[3-(1H-3-indolyl)-propyl]-1piperazinyl\} ethanamide. Compound (7d) as a Model

To a stirred solution of 3-(3-Piperazin-1-yl-propyl)-1H-indol 9a (224 mg; $0.92 \mathrm{mmol})$, in dry $\mathrm{CH}_{3} \mathrm{CN}$ (50 mL), 2-Chloro-1-(6-chloro-2,3-dihydro-benzo[b][1,4]oxazin-4-yl) ethanamide 4e (227 mg; $0.92 \mathrm{mmol})$, and anhydrous $\mathrm{K}_{2} \mathrm{CO}_{3}(127 \mathrm{mg} ; 0.92 \mathrm{mmol})$ were added. The mixture was heated at $80^{\circ} \mathrm{C}$ for $24 \mathrm{~h}$. After this time, the resulting mixture was poured into water $(100 \mathrm{~mL})$ and extracted with EtOAc $(4 \times 50 \mathrm{~mL})$, dried over anhydrous $\mathrm{Na}_{2} \mathrm{SO}_{4}$, and concentrated under reduced pressure. The organic crude was purified by column chromatography EtOAc/MeOH (6:1) to give pure $7 \mathbf{d}(290 \mathrm{mg}$; 71.6\% yield) as a yellow light solid. m.p.: $135.3-136.1{ }^{\circ} \mathrm{C} ;{ }^{1} \mathrm{H}-\mathrm{NMR}$ (DMSO- $\left.d_{6}\right): \delta 1.78\left(\mathrm{q}, 2 \mathrm{H}, \mathrm{H}-2^{\prime}\right.$, $J=7.0 \mathrm{~Hz}), 2.20-2.52\left(\mathrm{~m}, 10 \mathrm{H}, \mathrm{H}-3^{\prime}, \mathrm{H}-4^{\prime}\right.$ and $\left.\mathrm{H}-5^{\prime}\right), 2.68\left(\mathrm{t}, 2 \mathrm{H}, \mathrm{H}-1^{\prime}, J=7.3 \mathrm{~Hz}\right), 3.32\left(\mathrm{~s}, 2 \mathrm{H}, \mathrm{H}-7^{\prime}\right), 3.91$ $\left(\mathrm{t}, 2 \mathrm{H}, \mathrm{H}-3^{\prime \prime}, J=3.7 \mathrm{~Hz}\right), 4.27\left(\mathrm{t}, 2 \mathrm{H}, \mathrm{H}-2^{\prime \prime}, J=3.8 \mathrm{~Hz}\right), 6.90\left(\mathrm{~d}, 1 \mathrm{H}, \mathrm{H}-8^{\prime \prime}, J=8.8 \mathrm{~Hz}\right), 6.96(\mathrm{t}, 1 \mathrm{H}, \mathrm{H}-5$ or H-6, J = 7.3 Hz), 7.02-7.12 (m, 3H, 2-H, H-6 or H-5 and H-7" $), 7.34(\mathrm{~d}, 1 \mathrm{H}, \mathrm{H}-4, J=8.0 \mathrm{~Hz}), 7.50$ (d, $1 \mathrm{H}, \mathrm{H}-7, J=7.8 \mathrm{~Hz}$ ), 8.09 (br. s, $1 \mathrm{H}, \mathrm{H}-5^{\prime \prime}$ ), and 10.75 (br. s, $1 \mathrm{H}, \mathrm{H}-1$ ) ppm. ${ }^{13} \mathrm{C}-\mathrm{NMR}$ (DMSO- $d_{6}$ ): $\delta 22.9,27.6,39.3,40.5,52.9(2 \mathrm{X}), 53.2(2 \mathrm{X}), 58.0,66.4,111.8,114.9,118.5,118.6,118.7,121.2,122.6,123.5$, 123.6, 125.2, 127.6, 127.7, 136.8, 145.5, and 168.6 ppm. HRMS: (EI) Calculated for $\mathrm{C}_{25} \mathrm{H}_{29} \mathrm{ClN}_{4} \mathrm{O}_{2}\left(\mathrm{M}^{+}\right)$ $=453.9864$. Found: 453.9856 .

1-(6-Bromo-2,3-dihydro-benzo[b][1,4]oxazin-4-yl)-2-\{4-[3-(1H-3-indolyl)-propyl]-1-piperazinyl\} ethanamide (7e)

To a solution of 3-(3-Piperazin-1-yl-propyl)-1H-indol 9a (241 mg; $0.99 \mathrm{mmol})$ in $\mathrm{CH}_{3} \mathrm{CN}$ (50 mL), 2-Chloro-1-(6-Bromo-2,3-dihydro-benzo[b][1,4]oxazin-4-yl) ethanamide $4 \mathbf{d}$ (287 mg; $0.99 \mathrm{mmol})$ and anhydrous $\mathrm{K}_{2} \mathrm{CO}_{3}(137 \mathrm{mg} ; 0.99 \mathrm{mmol})$ were added to afford $7 \mathbf{e}(298 \mathrm{mg} ; 62.6 \%$ yield) as a yellow light 
solid. m.p.: $132.7-134.1^{\circ} \mathrm{C} ;{ }^{1} \mathrm{H}-\mathrm{NMR}$ (DMSO- $\left.d_{6}\right): \delta 1.77\left(\mathrm{q}, 2 \mathrm{H}, \mathrm{H}-2^{\prime}, J=6.7 \mathrm{~Hz}\right), 2.27-2.51(\mathrm{~m}, 10 \mathrm{H}$, H-3', H-4' and H-5'), 2.68 (t, 2H, H-1',$J=7.3 \mathrm{~Hz}), 3.32\left(\mathrm{~s}, 2 \mathrm{H}, \mathrm{H}-7^{\prime}\right), 3.91\left(\mathrm{~m}, 2 \mathrm{H}, \mathrm{H}-3^{\prime \prime}\right), 4.27(\mathrm{t}, 2 \mathrm{H}$, $\left.\mathrm{H}-2^{\prime \prime}, J=3.8 \mathrm{~Hz}\right), 6.86\left(\mathrm{~d}, 1 \mathrm{H}, \mathrm{H}-8^{\prime \prime}, J=8.8 \mathrm{~Hz}\right), 6.96\left(\mathrm{td}, 1 \mathrm{H}, \mathrm{H}-5\right.$ or H-6, $\left.J_{o}=7.3 \mathrm{~Hz}, J_{m}=1.0 \mathrm{~Hz}\right)$, 7.05 (td, 1H, H-6 or H-5, Jo = 7.3 Hz, $\left.J_{m}=1.0 \mathrm{~Hz}\right), 7.10$ (d, 1H, H-2, J = 1.9 Hz), 7.19 (dd, 1H, H-7" , $\left.J_{o}=8.7 \mathrm{~Hz}, J_{m}=2.1 \mathrm{~Hz}\right), 7.33(\mathrm{~d}, 1 \mathrm{H}, \mathrm{H}-4, J=8.0 \mathrm{~Hz}), 7.50(\mathrm{~d}, 1 \mathrm{H}, \mathrm{H}-7, J=7.8 \mathrm{~Hz}), 8.20($ br. $\mathrm{s}, 1 \mathrm{H}$, H-5"), and 10.77 (br.s, $1 \mathrm{H}, \mathrm{H}-1$ ) ppm. ${ }^{13} \mathrm{C}-\mathrm{NMR}$ (DMSO- $d_{6}$ ): $\delta 22.9,27.6,39.3,40.5,52.9(2 \mathrm{X}), 53.2(2 \mathrm{X})$, 58.0, 66.5, 111.1, 111.8, 114.8, 118.5, 118.7, 119.1, 121.2, 122.6, 125.4, 126.3, 127.6, 128.1, 136.7, 146.0, and 168.6 ppm. HRMS: (EI) Calculated for $\mathrm{C}_{25} \mathrm{H}_{29} \mathrm{BrN}_{4} \mathrm{O}_{2}\left(\mathrm{M}^{+}\right)=498.4373$. Found: 498.4364 .

1-(6-Methoxy-2,3-dihydro-benzo[b][1,4]oxazin-4-yl)-2-\{4-[3-(1H-3-indolyl)-propyl]-1-piperazinyl\} ethanamide (7f)

To a solution of 3-(3-Piperazin-1-yl-propyl)-1H-indol 9a (282 mg; $1.16 \mathrm{mmol})$ in $\mathrm{CH}_{3} \mathrm{CN}(50 \mathrm{~mL})$, 2-Chloro-1-(6-Methoxy-2,3-dihydro-benzo[b][1,4]oxazin-4-yl) ethanamide $4 \mathrm{f}$ (280 mg; $1.16 \mathrm{mmol}$ ) and anhydrous $\mathrm{K}_{2} \mathrm{CO}_{3}(160 \mathrm{mg} ; 1.16 \mathrm{mmol})$ were added to provide pure $7 \mathrm{f}(400 \mathrm{mg} ; 79 \%$ yield) as a yellow light solid. m.p.: $123.6-125.1^{\circ} \mathrm{C} ;{ }^{1} \mathrm{H}-\mathrm{NMR}$ (DMSO- $\left.d_{6}\right): \delta 1.75$ (q, $2 \mathrm{H}, \mathrm{H}-2^{\prime}, J=7.0 \mathrm{~Hz}$ ), $2.23-2.49$ (m, $10 \mathrm{H}, \mathrm{H}-3^{\prime}, \mathrm{H}-4^{\prime}$ and $\left.\mathrm{H}-5^{\prime}\right), 2.65\left(\mathrm{t}, 2 \mathrm{H}, \mathrm{H}-1^{\prime}, J=7.4 \mathrm{~Hz}\right), 3.31\left(\mathrm{~s}, 2 \mathrm{H}, \mathrm{H}-7^{\prime}\right), 3.65\left(\mathrm{~s}, 3 \mathrm{H}, \mathrm{H}-6^{\prime \prime}\right), 3.86(\mathrm{t}, 2 \mathrm{H}$, $\left.\mathrm{H}-3^{\prime \prime}, J=3.8 \mathrm{~Hz}\right), 4.18\left(\mathrm{t}, 2 \mathrm{H}, \mathrm{H}-2^{\prime \prime}, J=3.8 \mathrm{~Hz}\right), 6.63\left(\mathrm{dd}, 1 \mathrm{H}, \mathrm{H}-7^{\prime \prime}, J_{o}=8.9 \mathrm{~Hz}, J_{m}=2.3 \mathrm{~Hz}\right), 6.79(\mathrm{~d}$, $\left.1 \mathrm{H}, \mathrm{H}^{\prime \prime}, J=8.9 \mathrm{~Hz}\right), 6.94(\mathrm{t}, 1 \mathrm{H}, \mathrm{H}-5$ or H-6, $J=7.0 \mathrm{~Hz}), 7.03(\mathrm{t}, 1 \mathrm{H}, \mathrm{H}-6$ or H-5, $J=6.9 \mathrm{~Hz}), 7.08(\mathrm{~d}$, $1 \mathrm{H}, \mathrm{H}-2, J=1.9 \mathrm{~Hz}), 7.31$ (d, 1H, H-4, $J=8.0 \mathrm{~Hz}), 7.48$ (d, $1 \mathrm{H}, \mathrm{H}-7, J=7.8 \mathrm{~Hz}), 7.61$ (br. s, $\left.1 \mathrm{H}, \mathrm{H}-5^{\prime \prime}\right)$, and 10.74 (br. s, $1 \mathrm{H}, \mathrm{H}-1$ ) ppm. ${ }^{13} \mathrm{C}-\mathrm{NMR}$ (DMSO- $d_{6}$ ): $\delta 22.8,27.6,39.3,40.5,53.0(2 \mathrm{X}), 53.2(2 \mathrm{X}), 55.8$, 58.0, 66.1, 109.3, 111.7, 114.8, 117.3, 118.4, 118.7, 121.1, 122.5, 126.8, 127.4, 127.6, 136.7, 140.7, 152.6, and 168.3 ppm. HRMS: (EI) Calculated for $\mathrm{C}_{26} \mathrm{H}_{32} \mathrm{~N}_{4} \mathrm{O}_{3}\left(\mathrm{M}^{+}\right)=449.5673$. Found: 449.5669 .

1-(6-Chloro-2,3-dihydro-benzo[b][1,4]oxazin-4-yl)-2-\{4-[3-(5-fluoro-1H-3-indolyl)-propyl]-1-piperazinyl\} ethanamide $(7 \mathbf{j})$

To a solution of 5-Fluoro-3-(3-piperazin-1-yl-propyl)-1H-indol $9 \mathbf{b}(340 \mathrm{mg} ; 1.3 \mathrm{mmol})$ in $\mathrm{CH}_{3} \mathrm{CN}$ (50 mL), 2-Chloro-1-(6-chloro-2,3-dihydro-benzo[b][1,4]oxazin-4-yl) ethanamide 4e (320 mg; $1.3 \mathrm{mmol})$ and anhydrous $\mathrm{K}_{2} \mathrm{CO}_{3}(180 \mathrm{mg} 1.3 \mathrm{mmol}$ ) were added to afford pure compound $7 \mathbf{j}$ (540 mg; $91 \%$ yield) as a yellow light solid. m.p.: $120.5-122.8^{\circ} \mathrm{C} ;{ }^{1} \mathrm{H}-\mathrm{NMR}$ (DMSO- $\left.d_{6}\right): \delta 1.75\left(\mathrm{q}, 2 \mathrm{H}, \mathrm{H}-2^{\prime}, J=7.1 \mathrm{~Hz}\right.$ ), 2.21-2.50 (m, 10H, H-3' , H-4' and H-5'), $2.64\left(\mathrm{t}, 2 \mathrm{H}, \mathrm{H}-\mathrm{1}^{\prime}, J=7.4 \mathrm{~Hz}\right), 3.33\left(\mathrm{~s}, 2 \mathrm{H}, \mathrm{H}-7^{\prime}\right), 3.91(\mathrm{t}, 2 \mathrm{H}$, $\left.\mathrm{H}-3^{\prime \prime}, J=3.7 \mathrm{~Hz}\right), 4.27\left(\mathrm{t}, 2 \mathrm{H}, \mathrm{H}-2^{\prime \prime}, J=3.7 \mathrm{~Hz}\right), 6.85-6.92\left(\mathrm{~m}, 2 \mathrm{H}, \mathrm{H}-6\right.$ and H-8 $\left.{ }^{\prime \prime}\right), 7.07$ (dd, 1H, H-7" , $\left.J_{o}=8.7 \mathrm{~Hz}, J_{m}=2.3 \mathrm{~Hz}\right), 7.19(\mathrm{~d}, 1 \mathrm{H}, \mathrm{H}-2, J=1.7 \mathrm{~Hz}), 7.24\left(\mathrm{dd}, 1 \mathrm{H}, \mathrm{H}-7, \mathrm{~J}_{o}=10.2 \mathrm{~Hz}, J_{m}=2.3 \mathrm{~Hz}\right), 7.31$ (dd, $\left.1 \mathrm{H}, \mathrm{H}-4, J_{o}=8.9 \mathrm{~Hz}, J_{m}=4.7 \mathrm{~Hz}\right), 8.07$ (br. s, $\left.1 \mathrm{H}, \mathrm{H}-5^{\prime \prime}\right)$, and 10.88 (br. s, $\left.1 \mathrm{H}, \mathrm{H}-1\right)$ ppm. ${ }^{13} \mathrm{C}-\mathrm{NMR}$ (DMSO- $\left.d_{6}\right): \delta 22.7,27.5,39.3,40.5,52.9(2 X), 53.2(2 X), 57.8,66.4,103.4\left(\mathrm{~d},{ }^{2} J_{\mathrm{C}-\mathrm{F}}=23 \mathrm{~Hz}\right), 109.3\left(\mathrm{~d},{ }^{2}{ }^{\prime} \mathrm{C}_{\mathrm{C}-\mathrm{F}}\right.$ $=26.3 \mathrm{~Hz}), 112.6\left(\mathrm{~d},{ }^{3} J_{\mathrm{C}-\mathrm{F}}=9.9 \mathrm{~Hz}\right), 114.4,115.1\left(\mathrm{~d},{ }^{4} \mathrm{~J}_{\mathrm{C}-\mathrm{F}}=5.4 \mathrm{~Hz}\right), 118.7,123.5,124.8,125.2,127.5,127.9$ $\left(\mathrm{d},{ }^{3} J_{\mathrm{C}-\mathrm{F}}=9.3 \mathrm{~Hz}\right), 133.3,145.5,157.0\left(\mathrm{~d},{ }^{1} J_{\mathrm{C}-\mathrm{F}}=231 \mathrm{~Hz}\right)$, and $168.6 \mathrm{ppm}$. HRMS: (EI) Calculated for $\mathrm{C}_{25} \mathrm{H}_{28} \mathrm{ClFN}_{4} \mathrm{O}_{2}\left(\mathrm{M}^{+}\right)=471.9769$. Found: 471.9767 .

1-(6-Bromo-2,3-dihydro-benzo[b][1,4]oxazin-4-yl)-2-\{4-[3-(5-fluoro-1H-3-indolyl)-propyl]-1-piperazinyl\} ethanamide (7k)

To a solution of 5-Fluoro-3-(3-piperazin-1-yl-propyl)- $1 \mathrm{H}$-indol $9 \mathbf{b}$ (784 mg; $3 \mathrm{mmol}$ ) in $\mathrm{CH}_{3} \mathrm{CN}$ (50 $\mathrm{mL})$, 2-Chloro-1-(6-bromo-2,3-dihydro-benzo[b][1,4]oxazin-4-yl) ethanamide $4 \mathrm{~d}$ (870 $\mathrm{mg} ; 3 \mathrm{mmol}$ ) and anhydrous $\mathrm{K}_{2} \mathrm{CO}_{3}$ (180 mg $1.3 \mathrm{mmol}$ ) were added to afford pure compound $7 \mathbf{k}(1130 \mathrm{mg} ; 75.3 \%$ yield) as a yellow light solid. m.p.: $102.8-103.9^{\circ} \mathrm{C} ;{ }^{1} \mathrm{H}-\mathrm{NMR}$ (DMSO- $\left.d_{6}\right): \delta 1.80\left(\mathrm{q}, 2 \mathrm{H}, \mathrm{H}-2^{\prime}, J=7.0 \mathrm{~Hz}\right.$ ), 2.26-2.56 (m, 10H, H-3' , H-4 ${ }^{\prime}$ and H-5'), $2.69\left(\mathrm{t}, 2 \mathrm{H}, \mathrm{H}-\mathrm{-}^{\prime}, J=7.3 \mathrm{~Hz}\right), 3.38\left(\mathrm{~s}, 2 \mathrm{H}, \mathrm{H}-7^{\prime}\right), 3.97(\mathrm{t}, 2 \mathrm{H}$, $\left.\mathrm{H}-3^{\prime \prime}, J=3.4 \mathrm{~Hz}\right), 4.33\left(\mathrm{t}, 2 \mathrm{H}, \mathrm{H}-2^{\prime \prime}, J=3.6 \mathrm{~Hz}\right), 6.89-6.99(\mathrm{~m}, 2 \mathrm{H}, \mathrm{H}-6$, and H-8" $), 7.13$ (dd, 1H, H-7", $\left.J_{o}=8.7 \mathrm{~Hz}, J_{m}=2.3 \mathrm{~Hz}\right), 7.24(\mathrm{~d}, 1 \mathrm{H}, \mathrm{H}-2, J=1.9 \mathrm{~Hz}), 7.30\left(\mathrm{dd}, 1 \mathrm{H}, \mathrm{H}-7, J_{o}=10.2 \mathrm{~Hz}, J_{m}=2.3 \mathrm{~Hz}\right), 7.37$ $\left(\mathrm{dd}, 1 \mathrm{H}, \mathrm{H}-4, J_{o}=8.8 \mathrm{~Hz}, J_{m}=4.5 \mathrm{~Hz}\right), 8.13$ (br. s, $\left.1 \mathrm{H}, \mathrm{H}-5^{\prime \prime}\right)$, and 10.93 (br. s, $\left.1 \mathrm{H}, \mathrm{H}-1\right)$ ppm. ${ }^{13} \mathrm{C}-\mathrm{NMR}$ (DMSO- $\left.d_{6}\right): \delta 22.7,27.5,39.3,40.5,52.9(2 X), 53.2(2 \mathrm{X}), 57.9,66.4,103.4\left(\mathrm{~d},{ }^{2} J_{\mathrm{C}-\mathrm{F}}=23.1 \mathrm{~Hz}\right), 109.3(\mathrm{~d}$, $\left.{ }^{2} J_{\mathrm{C}-\mathrm{F}}=26 \mathrm{~Hz}\right), 112.6\left(\mathrm{~d},{ }^{3} J_{\mathrm{C}-\mathrm{F}}=9.9 \mathrm{~Hz}\right), 115.1\left(\mathrm{~d},{ }^{4} J_{\mathrm{C}-\mathrm{F}}=4.9 \mathrm{~Hz}\right), 118.7,123.4,123.5,124.8,125.3,127.6$, 
$127.9\left(\mathrm{~d},{ }^{3} J_{\mathrm{C}-\mathrm{F}}=9.3 \mathrm{~Hz}\right), 133.3,145.5,157.0\left(\mathrm{~d},{ }^{1} J_{\mathrm{C}-\mathrm{F}}=231 \mathrm{~Hz}\right)$, and $168.6 \mathrm{ppm}$. HRMS: (EI) Calculated for $\mathrm{C}_{25} \mathrm{H}_{28} \mathrm{BrFN}_{4} \mathrm{O}_{2}\left(\mathrm{M}^{+}\right)=516.4278$. Found: 516.4270.

1-(6-Methoxy-2,3-dihydro-benzo[b][1,4]oxazin-4-yl)-2-\{4-[3-(5-fluoro-1H-3-indolyl)-propyl]-1piperazinyl\} ethanamide (7l)

To a solution of 5-Fluoro-3-(3-piperazin-1-yl-propyl)-1H-indol $9 \mathrm{~b}$ (379 $\mathrm{mg} ; 1.45 \mathrm{mmol})$ in $\mathrm{CH}_{3} \mathrm{CN}$ (50 mL), 2-chloro-1-(6-methoxy-2,3-dihydro-benzo[b][1,4] oxazin-4-yl) ethanamide $4 \mathbf{f}$ (350 $\mathrm{mg} ; 1.45 \mathrm{mmol})$ and anhydrous $\mathrm{K}_{2} \mathrm{CO}_{3}(200 \mathrm{mg} ; 1.45 \mathrm{mmol})$ were added to afford pure compound 71 (535 mg; 81.6\% yield) as a yellow light solid. m.p.: $94.5-97.3^{\circ} \mathrm{C} ;{ }^{1} \mathrm{H}-\mathrm{NMR}$ (DMSO- $d_{6}$ ): $\delta 1.75$ (q, $\left.2 \mathrm{H}, \mathrm{H}-2^{\prime}, J=7.0 \mathrm{~Hz}\right), 2.2-2.50\left(\mathrm{~m}, 10 \mathrm{H}, \mathrm{H}-3^{\prime}, \mathrm{H}-4^{\prime}\right.$ and $\left.\mathrm{H}-5^{\prime}\right), 2.64\left(\mathrm{t}, 2 \mathrm{H}, \mathrm{H}-1^{\prime}, J=7.4 \mathrm{~Hz}\right), 3.34(\mathrm{~s}, 2 \mathrm{H}$,

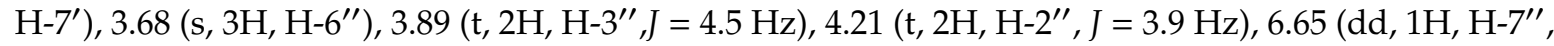
$\left.J_{o}=8.9 \mathrm{~Hz}, J_{m}=2.9 \mathrm{~Hz}\right), 6.81\left(\mathrm{~d}, 1 \mathrm{H}, \mathrm{H}-8^{\prime \prime}, J=8.9 \mathrm{~Hz}\right), 6.89\left(\mathrm{td}, 1 \mathrm{H}, \mathrm{H}-6, J_{o}=9.2 \mathrm{~Hz}, J_{m}=2.5 \mathrm{~Hz}\right), 7.19$ $(\mathrm{d}, 1 \mathrm{H}, \mathrm{H}-2, J=2.2 \mathrm{~Hz}), 7.25\left(\mathrm{dd}, 1 \mathrm{H}, \mathrm{H}-7, J_{o}=10.1 \mathrm{~Hz}, J_{m}=2.5 \mathrm{~Hz}\right), 7.32\left(\mathrm{dd}, 1 \mathrm{H}, \mathrm{H}-4, J_{o}=8.8 \mathrm{~Hz}, J_{m}\right.$ $=4.6 \mathrm{~Hz}$ ), 7.62 (br. s, $1 \mathrm{H}, \mathrm{H}-5^{\prime \prime}$ ), and 10.88 (br. s, $1 \mathrm{H}, \mathrm{H}-1$ ) ppm. ${ }^{13} \mathrm{C}-\mathrm{NMR}$ (DMSO- $d_{6}$ ): $\delta$ 22.7, 27.6, 39.3, 40.5, $53.1(2 \mathrm{X}), 53.3(2 \mathrm{X}), 55.9,57.9,66.2,103.4\left(\mathrm{~d},{ }^{2} J_{\mathrm{C}-\mathrm{F}}=23.2 \mathrm{~Hz}\right), 109.3\left(\mathrm{~d},{ }^{2} J_{\mathrm{C}-\mathrm{F}}=26 \mathrm{~Hz}\right), 109.3$, $112.6\left(\mathrm{~d},{ }^{3} J_{\mathrm{C}-\mathrm{F}}=10 \mathrm{~Hz}\right), 115.2\left(\mathrm{~d},{ }^{4} J_{\mathrm{C}-\mathrm{F}}=4.4 \mathrm{~Hz}\right), 117.4,124.9,126.9,127.9\left(\mathrm{~d},{ }^{3^{\prime}} J_{\mathrm{C}-\mathrm{F}}=10 \mathrm{~Hz}\right), 128.6$, 133.4, 140.7, 152.7, $157.0\left(\mathrm{~d},{ }^{1} J_{\mathrm{C}-\mathrm{F}}=231 \mathrm{~Hz}\right)$, and $168.4 \mathrm{ppm}$. HRMS: (EI) Calculated for $\mathrm{C}_{26} \mathrm{H}_{31} \mathrm{FN}_{4} \mathrm{O}_{3}$ $\left(\mathrm{M}^{+}\right)=467.5577$. Found: 467.5567.

3.1.5. General Procedure for the Synthesis of N-(2-morpholin-4-yl-ethyl)-benzamides nitro-fluorinated Derivatives 10a-d

4-Fluoro-N-(2-morpholin-4-yl-ethyl)-2-nitro-benzamide (10a) as a Model

To a solution of 4-Fluoro-2-nitro-benzoyl chloride ( $828 \mathrm{mg}$; $4.07 \mathrm{mmol})$ in dry THF $(50 \mathrm{~mL})$, 2-morpholin-4-yl-ethylamine $(0.53 \mathrm{~mL} ; 4.07 \mathrm{mmol})$ was added. The mixture was stirred at $0{ }^{\circ} \mathrm{C}$ under nitrogen atmosphere for $2 \mathrm{~h}$. After this time, saturated solution of $\mathrm{NaHCO}_{3}$ was added $(100 \mathrm{~mL})$ and the mixture was extracted with EtOAc $(3 \times 60 \mathrm{~mL})$. The organic layers were dried over anhydrous $\mathrm{Na}_{2} \mathrm{SO}_{4}$ filtered, and concentrated under vacuum conditions to give a crude form, which was purified by column chromatography with $\mathrm{EtOAc} / \mathrm{MeOH}(6: 1)$ as eluent to give 10 a ( $963 \mathrm{mg} ; 80 \%$ yield) as a yellow light solid. m.p.: $149.8-151.5^{\circ} \mathrm{C} ;{ }^{1} \mathrm{H}-\mathrm{NMR}$ (acetone- $\left.d_{6}\right): \delta 2.32\left(\mathrm{t}, 4 \mathrm{H}, \mathrm{H}-4^{\prime}, J=4.6 \mathrm{~Hz}\right), 2.42$ $\left(\mathrm{t}, 2 \mathrm{H}, \mathrm{H}-3^{\prime}, J=6.3 \mathrm{~Hz}\right), 3.35\left(\mathrm{q}, 2 \mathrm{H}, \mathrm{H}-2^{\prime}, J=6.3 \mathrm{~Hz}\right), 3.46\left(\mathrm{t}, 4 \mathrm{H}, \mathrm{H}-5^{\prime}, J=4.5 \mathrm{~Hz}\right), 7.45(\mathrm{td}, 1 \mathrm{H}, \mathrm{H}-5$, $\left.J_{o}=8.2 \mathrm{~Hz}, J_{m}=2.5 \mathrm{~Hz}\right), 7.59\left(\mathrm{dd}, 1 \mathrm{H}, \mathrm{H}-6, J_{o}=8.5 \mathrm{~Hz}, J_{m}=5.5 \mathrm{~Hz}\right), 7.65$ (br. s, $\left.1 \mathrm{H}, \mathrm{H}-1^{\prime}\right)$, and 7.70 $\left(\mathrm{dd}, 1 \mathrm{H}, \mathrm{H}-3, J_{o}=8.5 \mathrm{~Hz}, J_{m}=2.6 \mathrm{~Hz}\right) \mathrm{ppm} .{ }^{13} \mathrm{C}-\mathrm{NMR}$ (acetone- $\left.d_{6}\right): \delta 36.1,53(2 \mathrm{X}), 56.6,66(2 \mathrm{X}), 111.3$ $\left(\mathrm{d}, 2 J_{\mathrm{C}-\mathrm{F}}=27 \mathrm{~Hz}\right), 119.6\left(\mathrm{~d}, 2^{\prime} J_{\mathrm{C}-\mathrm{F}}=22 \mathrm{~Hz}\right), 129.1\left(\mathrm{~d}, 4 J_{\mathrm{C}-\mathrm{F}}=3.3 \mathrm{~Hz}\right), 130.5\left(\mathrm{~d}, 3 J_{\mathrm{C}-\mathrm{F}}=9 \mathrm{~Hz}\right), 148.1(\mathrm{~d}$, $\left.3^{\prime} J_{\mathrm{C}-\mathrm{F}}=8.2 \mathrm{~Hz}\right), 161.7\left(\mathrm{~d}, 1 J_{\mathrm{C}-\mathrm{F}}=251 \mathrm{~Hz}\right)$, and $164.2 \mathrm{ppm}$. HRMS: (EI) Calculated for $\mathrm{C}_{13} \mathrm{H}_{16} \mathrm{FN}_{3} \mathrm{O}_{4}$ $\left(\mathrm{M}^{+}\right)=298.1203$. Found: 298.1198 .

4-Fluoro-N-(2-morpholin-4-yl-ethyl)-3-nitro-benzamide (10b)

4-Fluoro-3-nitro-benzoyl chloride ( $553 \mathrm{mg}$; $2.72 \mathrm{mmol}$ ), and 2-morpholin-4-yl-ethylamine $(0.35 \mathrm{~mL}$; $2.72 \mathrm{mmol})$, to afford $\mathbf{1 0 b}$ (712 mg; $88 \%$ yield) as a yellow light solid. m.p.: $132.5-133.5^{\circ} \mathrm{C} ;{ }^{1} \mathrm{H}-\mathrm{NMR}$ (acetone- $\left.d_{6}\right): \delta 2.32\left(\mathrm{t}, 4 \mathrm{H}, \mathrm{H}-4^{\prime}, J=4.4 \mathrm{~Hz}\right), 2.43\left(\mathrm{t}, 2 \mathrm{H}, \mathrm{H}-3^{\prime}, J=6.6 \mathrm{~Hz}\right), 3.37-3.50\left(\mathrm{~m}, 6 \mathrm{H}, \mathrm{H}-2^{\prime}\right.$ and H-5'), 7.48 (m, 1H, H-5), 7.92 (br. s, 1H, H-1'), 8.16 (m, 1H, H-2), and 8.45 (dd, $1 \mathrm{H}, \mathrm{H}-6, J_{o}=7.2 \mathrm{~Hz}$, $\left.J_{m}=2.3 \mathrm{~Hz}\right)$ ppm. ${ }^{13} \mathrm{C}-\mathrm{NMR}\left(\right.$ acetone- $\left.d_{6}\right): \delta 36.4,53.1(2 \mathrm{X}), 56.9,66.1(2 \mathrm{X}), 118.1\left(\mathrm{~d},{ }^{2} J_{\mathrm{C}-\mathrm{F}}=21.4 \mathrm{~Hz}\right)$, $124.6\left(\mathrm{~d},{ }^{4} J_{\mathrm{C}-\mathrm{F}}=1.6 \mathrm{~Hz}\right), 131.6\left(\mathrm{~d},{ }^{3} J_{\mathrm{C}-\mathrm{F}}=3.8 \mathrm{~Hz}\right), 134.2\left(\mathrm{~d},{ }^{2} J_{\mathrm{C}-\mathrm{F}}=9.9 \mathrm{~Hz}\right), 150.4\left(\mathrm{~d},{ }^{3^{\prime}} J_{\mathrm{C}-\mathrm{F}}=3.9 \mathrm{~Hz}\right)$, $156.2\left(\mathrm{~d},{ }^{1} \mathrm{~J}_{\mathrm{C}-\mathrm{F}}=266 \mathrm{~Hz}\right)$, and $163 \mathrm{ppm}$. HRMS: (EI) Calculated for $\mathrm{C}_{13} \mathrm{H}_{16} \mathrm{FN}_{3} \mathrm{O}_{4}\left(\mathrm{M}^{+}\right)=298.1203$, Found: 298.1201.

\section{5-Fluoro- $N$-(2-morpholin-4-yl-ethyl)-2-nitro-benzamide (10c)}

5-Fluoro-2-nitro-benzoyl chloride ( $803 \mathrm{mg}$; $3.94 \mathrm{mmol})$, and 2-morpholin-4-yl-ethylamine $(0.51 \mathrm{~mL}$; $3.94 \mathrm{mmol})$, to afford $10 \mathrm{c}\left(951 \mathrm{mg} ; 81 \%\right.$ yield) as a yellow light solid. m.p.: $103.4-104.5^{\circ} \mathrm{C} ;{ }^{1} \mathrm{H}-\mathrm{NMR}$ 
(acetone- $\left.d_{6}\right): \delta 2.33\left(\mathrm{t}, 4 \mathrm{H}, \mathrm{H}-4^{\prime}, J=4.6 \mathrm{~Hz}\right), 2.44\left(\mathrm{t}, 2 \mathrm{H}, \mathrm{H}-3^{\prime}, J=6.3 \mathrm{~Hz}\right), 3.36\left(\mathrm{~m}, 2 \mathrm{H}, \mathrm{H}-2^{\prime}\right), 3.46(\mathrm{t}, 4 \mathrm{H}$, H-5',$J=4.5 \mathrm{~Hz}$ ), 7.23-7.37 (m, 2H, H-3, and H-4), 7.67 (br. s, $\left.1 \mathrm{H}, \mathrm{H}-1^{\prime}\right)$, and $8.02\left(\mathrm{dd}, 1 \mathrm{H}, \mathrm{H}-6, J_{o}=9.1\right.$ $\left.\mathrm{Hz}, J_{m}=4.8 \mathrm{~Hz}\right)$ ppm. ${ }^{13} \mathrm{C}-\mathrm{NMR}\left(\right.$ acetone- $\left.d_{6}\right): \delta 36.1,53(2 \mathrm{X}), 56.5,66(2 \mathrm{X}), 115.6\left(\mathrm{~d},{ }^{2} J_{\mathrm{C}-\mathrm{F}}=25.3 \mathrm{~Hz}\right)$, $116.5\left(\mathrm{~d},{ }^{2} J_{\mathrm{C}-\mathrm{F}}=23.6 \mathrm{~Hz}\right), 126.8\left(\mathrm{~d},{ }^{3} J_{\mathrm{C}-\mathrm{F}}=9.9 \mathrm{~Hz}\right), 136.0\left(\mathrm{~d},{ }^{3} J_{\mathrm{C}-\mathrm{F}}=8.8 \mathrm{~Hz}\right), 143\left(\mathrm{~d},{ }^{4} J_{\mathrm{C}-\mathrm{F}}=3.3 \mathrm{~Hz}\right)$, 163.9, and $163.9\left(\mathrm{~d},{ }^{1} J_{\mathrm{C}-\mathrm{F}}=256 \mathrm{~Hz}\right)$ ppm. HRMS: (EI) Calculated for $\mathrm{C}_{13} \mathrm{H}_{16} \mathrm{FN}_{3} \mathrm{O}_{4}\left(\mathrm{M}^{+}\right)=298.1203$. Found: 298.1201.

2-Fluor-N-(2-morpholin-4-yl-ethyl)-5-nitro-benzamide (10d)

2-Fluoro-5-nitro-benzoyl chloride ( $825 \mathrm{mg} ; 4.05 \mathrm{mmol})$, and 2-morpholin-4-yl-ethylamine $(0.53 \mathrm{~mL}$; $4.05 \mathrm{mmol})$, to afford $10 \mathrm{~d}\left(902 \mathrm{mg} ; 75 \%\right.$ yield) as a yellow light solid. m.p.: $126.4-127.2{ }^{\circ} \mathrm{C} ;{ }^{1} \mathrm{H}-\mathrm{NMR}$ (acetone- $\left.d_{6}\right): \delta 2.35\left(\mathrm{t}, 4 \mathrm{H}, \mathrm{H}-4^{\prime}, J=4.5 \mathrm{~Hz}\right), 2.46\left(\mathrm{t}, 2 \mathrm{H}, \mathrm{H}-3^{\prime}, J=6.3 \mathrm{~Hz}\right), 3.43\left(\mathrm{q}, 2 \mathrm{H}, \mathrm{H}-2^{\prime}, J=6.5 \mathrm{~Hz}\right)$, 3.49 (t, 4H, H-5',$J=4.5 \mathrm{~Hz}), 7.43$ (m, 1H, H-3), 7.66 (br. s, 1H, H-1'), 8.30 (m, 1H, H-4), and 8.56 (dd, $1 \mathrm{H}, \mathrm{H}-6, J_{o}=6.3 \mathrm{~Hz}, J_{m}=3 \mathrm{~Hz}$ ) ppm. ${ }^{13} \mathrm{C}-\mathrm{NMR}$ (acetone- $\left.d_{6}\right): \delta 36.1,52.9(2 \mathrm{X}), 56.2,66.1(2 \mathrm{X}), 117.4$ $\left(\mathrm{d},{ }^{2} J_{\mathrm{C}-\mathrm{F}}=26.9 \mathrm{~Hz}\right), 123.6\left(\mathrm{~d},{ }^{2} J_{\mathrm{C}-\mathrm{F}}=16.5 \mathrm{~Hz}\right), 126.3\left(\mathrm{~d},{ }^{4} J_{\mathrm{C}-\mathrm{F}}=4.9 \mathrm{~Hz}\right), 127.5\left(\mathrm{~d},{ }^{3} J_{\mathrm{C}-\mathrm{F}}=11 \mathrm{~Hz}\right), 144(\mathrm{~d}$, $\left.{ }^{3} J_{\mathrm{C}-\mathrm{F}}=9 \mathrm{~Hz}\right), 160.4$, and $162.8\left(\mathrm{~d},{ }^{1} J_{\mathrm{C}-\mathrm{F}}=259 \mathrm{~Hz}\right)$ ppm. HRMS: (EI) Calculated for $\mathrm{C}_{13} \mathrm{H}_{16} \mathrm{FN}_{3} \mathrm{O}_{4}$ $\left(\mathrm{M}^{+}\right)=298.1203$. Found: 298.1201.

3.1.6. General Procedure for the Synthesis of N-(2-morpholin-4-yl-ethyl)-benzamides amino-fluorinated Derivatives 11a-d

\section{2-Amino-N-(2-morpholin-4-yl-ethyl)-4-fluoro-benzamide (11a) as a Model}

To a mixture containing water-acetic acid-ethanol (1:1:1), 4-Fluoro- $N$-(2-morpholin-4-yl-ethyl)-2nitro-benzamide 10a (1 g; $3.36 \mathrm{mmol})$ and iron powder $(734 \mathrm{mg} ; 13.1 \mathrm{mmol})$ were added. The resulting mixture was heated and stirred for $3 \mathrm{~h}$ at $70^{\circ} \mathrm{C}$. After this time, the mixture was filtered to remove excess metallic iron, transferred to a flask containing a mixture of EtOAc/ $\mathrm{H}_{2} \mathrm{O}(400 \mathrm{~mL}, 1: 1)$, and neutralized with $\mathrm{NaHCO}_{3}(10 \mathrm{gr})$. The aqueous phase was extracted with EtOAc $(50 \mathrm{~mL} \times 3)$. The organic layer was dried over anhydrous $\mathrm{Na}_{2} \mathrm{SO}_{4}$ and concentrated under vacuum to give a crude, which was purified by column chromatography with EtOAc/MeOH (6:1) to give 11a (891 $\mathrm{mg}$; $98 \%$ yield) as a yellow light solid. m.p.: $120.3-121 .{ }^{\circ} \mathrm{C}$; ${ }^{1} \mathrm{H}-\mathrm{NMR}\left(\mathrm{CDCl}_{3}\right): \delta 2.46\left(\mathrm{t}, 4 \mathrm{H}, \mathrm{H}-4^{\prime}, J=4.4 \mathrm{~Hz}\right), 2.55\left(\mathrm{t}, 2 \mathrm{H}, \mathrm{H}-3^{\prime}\right.$, $J=6.1 \mathrm{~Hz}), 3.46\left(\mathrm{q}, 2 \mathrm{H}, \mathrm{H}-2^{\prime}, J=5.6 \mathrm{~Hz}\right), 3.69\left(\mathrm{t}, 4 \mathrm{H}, \mathrm{H}-5^{\prime}, J=4.7 \mathrm{~Hz}\right), 5.72$ (br. s, 2H, H-2), 6.28-6.35 (m, 2H, H-3 and H-5), 6.57 (br. s, 1H, H-1'), and 7.25 (dd, 1H, H-6, Jo $=9.3 \mathrm{~Hz}, J_{m}=3.2 \mathrm{~Hz}$ ) ppm. ${ }^{13} \mathrm{C}-\mathrm{NMR}\left(\mathrm{CDCl}_{3}\right): \delta 36.7,53.3(2 \mathrm{X}), 56.7,66.9(2 \mathrm{X}), 102.3\left(\mathrm{~d},{ }^{2} J_{\mathrm{C}-\mathrm{F}}=24.2 \mathrm{~Hz}\right), 103.9\left(\mathrm{~d},{ }^{\prime} J_{\mathrm{C}-\mathrm{F}}=23 \mathrm{~Hz}\right)$, $112.4\left(\mathrm{~d},{ }^{4} J_{\mathrm{C}-\mathrm{F}}=2.2 \mathrm{~Hz}\right), 129.3\left(\mathrm{~d},{ }^{3} J_{\mathrm{C}-\mathrm{F}}=11 \mathrm{~Hz}\right), 151.1\left(\mathrm{~d},{ }^{3} J_{\mathrm{C}-\mathrm{F}}=11.7 \mathrm{~Hz}\right), 165.3\left(\mathrm{~d},{ }^{1} J_{\mathrm{C}-\mathrm{F}}=249 \mathrm{~Hz}\right)$, and 168.6 ppm. HRMS: (EI) Calculated for $\mathrm{C}_{13} \mathrm{H}_{18} \mathrm{FN}_{3} \mathrm{O}_{2}\left(\mathrm{M}^{+}\right)=268.1461$. Found: 268.1454.

3-Amino-N-(2-morpholin-4-yl-ethyl)-4-fluoro-benzamide (11b)

4-Fluoro-N-(2-morpholin-4-yl-ethyl)-3-nitro-benzamide $\mathbf{1 0 b}(1 \mathrm{~g} ; 3.36 \mathrm{mmol})$ and iron powder (734 mg; $13.1 \mathrm{mmol})$ to afford $\mathbf{1 1 b}\left(726 \mathrm{mg} ; 81 \%\right.$ yield) as a yellow light solid. m.p.: $147.4-149.1^{\circ} \mathrm{C}$; ${ }^{1} \mathrm{H}-\mathrm{NMR}$ (DMSO-d 6 ): $\delta$ 2.40-2.51 (m, 4H, H-4'), 2.57 (br. s, 2H, H-3'), 3.40 (q, 2H, H-2', J = 6.6 Hz), $3.63\left(\mathrm{t}, 4 \mathrm{H}, \mathrm{H}-5^{\prime}, \mathrm{J}=4.3 \mathrm{~Hz}\right.$ ), 5.39 (br. s, 2H, H-3), 6.99-7.15 (m, 2H, H-2 and H-4), 7.31 (dd, 1H, H-6, $\left.J_{o}=8.9 \mathrm{~Hz}, J_{m}=1.6 \mathrm{~Hz}\right)$, and $6.57\left(\right.$ br. $\left.\mathrm{t}, 1 \mathrm{H}, \mathrm{H}-1^{\prime}, J=5.2 \mathrm{~Hz}\right), \mathrm{ppm} .{ }^{13} \mathrm{C}-\mathrm{NMR}\left(\mathrm{DMSO}-d_{6}\right): \delta 36.9$, $53.7(2 X), 57.8,66.6(2 X), 115.0\left(\mathrm{~d},{ }^{2} J_{\mathrm{C}-\mathrm{F}}=30 \mathrm{~Hz}\right), 115.1\left(\mathrm{~d},{ }^{3} J_{\mathrm{C}-\mathrm{F}}=3.3 \mathrm{~Hz}\right), 116\left(\mathrm{~d},{ }^{3} J_{\mathrm{C}-\mathrm{F}}=6 \mathrm{~Hz}\right), 131.7$ $\left(\mathrm{d},{ }^{4} J_{\mathrm{C}-\mathrm{F}}=2.8 \mathrm{~Hz}\right), 136.7\left(\mathrm{~d},{ }^{2} J_{\mathrm{C}-\mathrm{F}}=13.2 \mathrm{~Hz}\right), 152.5\left(\mathrm{~d},{ }^{1} J_{\mathrm{C}-\mathrm{F}}=242 \mathrm{~Hz}\right)$, and $168.3 \mathrm{ppm}$. HRMS: (EI) Calculated for $\mathrm{C}_{13} \mathrm{H}_{18} \mathrm{FN}_{3} \mathrm{O}_{2}\left(\mathrm{M}^{+}\right)=268.1461$. Found: 268.1458 .

\section{2-Amino-N-(2-morpholin-4-yl-ethyl)-5-fluoro-benzamide (11c)}

5-Fluoro-N-(2-morpholin-4-yl-ethyl)-2-nitro-benzamide 10c (1 g; $3.36 \mathrm{mmol})$ and iron powder (734 mg; $13.1 \mathrm{mmol})$ to afford 11c (697 mg; 78\% yield) as a yellow light solid. m.p.: $104.8-105.6{ }^{\circ} \mathrm{C}$; ${ }^{1} \mathrm{H}-\mathrm{NMR}\left(\mathrm{CDCl}_{3}\right): \delta 2.47\left(\mathrm{t}, 4 \mathrm{H}, \mathrm{H}-4^{\prime}, \mathrm{J}=4.4 \mathrm{~Hz}\right), 2.55\left(\mathrm{t}, 2 \mathrm{H}, \mathrm{H}-3^{\prime}, \mathrm{J}=6.1 \mathrm{~Hz}\right), 3.47\left(\mathrm{q}, 2 \mathrm{H}, \mathrm{H}-2^{\prime}\right.$, $J=5.6 \mathrm{~Hz}$ ), 3.69 (t, 4H, H-5',$J=4.6 \mathrm{~Hz}$ ), 5.28 (br. s, 2H, H-2), 6.55-6.63 (br. m, 2H, H-3, and H-1'), 
$6.93\left(\mathrm{td}, 1 \mathrm{H}, \mathrm{H}-6, J_{o}=8.6 \mathrm{~Hz}, J_{m}=2.9 \mathrm{~Hz}\right)$, and $7.0\left(\mathrm{dd}, 1 \mathrm{H}, \mathrm{H}-4, J_{o}=9.1 \mathrm{~Hz}, J_{m}=2.7 \mathrm{~Hz}\right) \mathrm{ppm}$. ${ }^{13} \mathrm{C}-\mathrm{NMR}\left(\mathrm{CDCl}_{3}\right): \delta 35.9,53.3(2 \mathrm{X}), 56.8,67(2 \mathrm{X}), 113.1\left(\mathrm{~d},{ }^{2} J_{\mathrm{C}-\mathrm{F}}=23 \mathrm{~Hz}\right), 116.6\left(\mathrm{~d},{ }^{3} J_{\mathrm{C}-\mathrm{F}}=5.1 \mathrm{~Hz}\right), 118.4$ $\left(\mathrm{d},{ }^{3} J_{\mathrm{C}-\mathrm{F}}=7.3 \mathrm{~Hz}\right), 119.5\left(\mathrm{~d},{ }^{2} J_{\mathrm{C}-\mathrm{F}}=23 \mathrm{~Hz}\right), 144.8\left(\mathrm{~d},{ }^{4} J_{\mathrm{C}-\mathrm{F}}=1.5 \mathrm{~Hz}\right)$, and $155.5\left(\mathrm{~d},{ }^{1} J_{\mathrm{C}-\mathrm{F}}=236 \mathrm{~Hz}\right)$, 168.3 ppm. HRMS: (EI) Calculated for $\mathrm{C}_{13} \mathrm{H}_{18} \mathrm{FN}_{3} \mathrm{O}_{2}\left(\mathrm{M}^{+}\right)=268.1461$. Found: 268.1459.

5-Amino- $N$-(2-morpholin-4-yl-ethyl)-2-fluoro-benzamide (11d)

2-Fluoro- $N$-(2-morpholin-4-yl-ethyl)-5-nitro-benzamide $10 \mathrm{~d}(1 \mathrm{~g} ; 3.36 \mathrm{mmol})$ and iron powder (734 mg; $13.1 \mathrm{mmol}$ ) to afford $11 \mathrm{~d}\left(798 \mathrm{mg}\right.$; $89 \%$ yield) as a yellow light solid. m.p.: 106.8-107.9 ${ }^{\circ} \mathrm{C}$; ${ }^{1} \mathrm{H}-\mathrm{NMR}\left(\mathrm{CDCl}_{3}\right): \delta 2.37\left(\mathrm{t}, 4 \mathrm{H}, \mathrm{H}-4^{\prime}, J=4.4 \mathrm{~Hz}\right), 2.45\left(\mathrm{t}, 2 \mathrm{H}, \mathrm{H}-3^{\prime}, J=6.1 \mathrm{~Hz}\right), 3.24-3.50$ (br. m, 4H, H-5 H-2'), 3.59 (t, 4H, H-5' , J = 4.7 Hz), $6.59(\mathrm{~m}, 1 \mathrm{H}, \mathrm{H}-6), 6.78$ (m, 1H, H-3), $7.23\left(\mathrm{dd}, 1 \mathrm{H}, \mathrm{H}-4, J_{o}=6.4 \mathrm{~Hz}\right.$, $J_{m}=3.2 \mathrm{~Hz}$ ), and 7.28 (br. s, $\left.1 \mathrm{H}, \mathrm{H}-1^{\prime}\right)$ ppm. ${ }^{13} \mathrm{C}-\mathrm{NMR}\left(\mathrm{CDCl}_{3}\right): \delta 36.3,53.2(2 \mathrm{X}), 56.5,67(2 \mathrm{X}), 116.6$ $\left(\mathrm{d},{ }^{2} J_{\mathrm{C}-\mathrm{F}}=26.4 \mathrm{~Hz}\right), 117.0\left(\mathrm{~d},{ }^{4} J_{\mathrm{C}-\mathrm{F}}=1.5 \mathrm{~Hz}\right), 119.1\left(\mathrm{~d},{ }^{3} J_{\mathrm{C}-\mathrm{F}}=8.8 \mathrm{~Hz}\right), 121.3\left(\mathrm{~d},{ }^{2} J_{\mathrm{C}-\mathrm{F}}=13.2 \mathrm{~Hz}\right), 143.1$ $\left(\mathrm{d},{ }^{3 \prime} J_{\mathrm{C}-\mathrm{F}}=2.2 \mathrm{~Hz}\right), 154.2\left(\mathrm{~d},{ }^{1} J_{\mathrm{C}-\mathrm{F}}=238 \mathrm{~Hz}\right)$, and $163.4 \mathrm{ppm}$. HRMS: (EI) Calculated for $\mathrm{C}_{13} \mathrm{H}_{18} \mathrm{FN}_{3} \mathrm{O}_{2}$ $\left(\mathrm{M}^{+}\right)=268.1461$. Found: 268.1457 .

3.1.7. General Procedure for the Synthesis of (2-Chloro-acetylamino)-N-(2-morpholin-4-yl-ethyl) fluorinated Benzamides Derivatives 12a-d

2-(2-Chloro-acetylamino)-4-fluoro-N-(2-morpholin-4-yl-ethyl)-benzamide (12a) as a Model

To a solution of 2-amino- $N$-(2-morpholin-4-yl-ethyl)-4-fluoro-benzamide 11a (400 mg; $1.5 \mathrm{mmol}$ ) in dry THF $(60 \mathrm{~mL}), 2$-chloro-acetylchloride $(0.12 \mathrm{~mL} ; 1.5 \mathrm{mmol})$ was added. The mixture was stirred at $0{ }^{\circ} \mathrm{C}$ under nitrogen atmosphere for $2 \mathrm{~h}$. After this time, a saturated solution of $\mathrm{NaHCO}_{3}(100$ $\mathrm{mL})$ was added. The mixture was extracted with EtOAc $(3$ X $100 \mathrm{~mL})$. The organic layers were dried over anhydrous $\mathrm{Na}_{2} \mathrm{SO}_{4}$, filtered, and concentrated under vacuum conditions to give a crude, which was purified by column chromatography EtOAc/MeOH (6:1), to give 12a (479 $\mathrm{mg}$; $93 \%$ yield) as a white solid. m.p.: $124.8-126.3^{\circ} \mathrm{C} ;{ }^{1} \mathrm{H}-\mathrm{NMR}\left(\mathrm{CDCl}_{3}\right): \delta 2.48\left(\mathrm{t}, 4 \mathrm{H}, \mathrm{H}-4^{\prime}, J=4.4 \mathrm{~Hz}\right), 2.58\left(\mathrm{t}, 2 \mathrm{H}, \mathrm{H}-3^{\prime}\right.$, $J=5.9 \mathrm{~Hz}), 3.51\left(\mathrm{q}, 2 \mathrm{H}, \mathrm{H}-2^{\prime}, J=5.4 \mathrm{~Hz}\right), 3.69\left(\mathrm{t}, 4 \mathrm{H}, \mathrm{H}-5^{\prime}, J=4.6 \mathrm{~Hz}\right), 4.13\left(\mathrm{~s}, 2 \mathrm{H}, \mathrm{H}-1^{\prime \prime}\right), 6.81(\mathrm{td}, 1 \mathrm{H}$, $\left.\mathrm{H}-5, J_{o}=7.7 \mathrm{~Hz}, J_{m}=2.7 \mathrm{~Hz}\right), 6.88\left(\mathrm{br} . \mathrm{s}, 1 \mathrm{H}, \mathrm{H}-\mathrm{1}^{\prime}\right), 7.46\left(\mathrm{dd}, 1 \mathrm{H}, \mathrm{H}-5, J_{o}=8.8 \mathrm{~Hz}, J_{m}=6.1 \mathrm{~Hz}\right), 8.42(\mathrm{dd}$, $1 \mathrm{H}, \mathrm{H}-3, J_{o}=11.6 \mathrm{~Hz}, J_{m}=2.7 \mathrm{~Hz}$ ), and 12.24 (br. s, $\left.1 \mathrm{H}, \mathrm{H}-2\right)$ ppm. ${ }^{13} \mathrm{C}-\mathrm{NMR}\left(\mathrm{CDCl}_{3}\right): \delta 36,43.2,53.3$ $(2 \mathrm{X}), 56.5,66.9(2 \mathrm{X}), 108.7\left(\mathrm{~d},{ }^{2} J_{\mathrm{C}-\mathrm{F}}=28 \mathrm{~Hz}\right), 110.6\left(\mathrm{~d},{ }^{2} J_{\mathrm{C}-\mathrm{F}}=22 \mathrm{~Hz}\right), 116.8\left(\mathrm{~d},{ }^{4} J_{\mathrm{C}-\mathrm{F}}=3.7 \mathrm{~Hz}\right), 128.4(\mathrm{~d}$, $\left.{ }^{3} J_{\mathrm{C}-\mathrm{F}}=10.3 \mathrm{~Hz}\right), 141\left(\mathrm{~d},{ }^{3^{\prime}} J_{\mathrm{C}-\mathrm{F}}=12.5 \mathrm{~Hz}\right), 164.7\left(\mathrm{~d},{ }^{1} J_{\mathrm{C}-\mathrm{F}}=252 \mathrm{~Hz}\right), 165.5$, and $167.8 \mathrm{ppm}$. HRMS: (EI) Calculated for $\mathrm{C}_{15} \mathrm{H}_{19} \mathrm{ClFN}_{3} \mathrm{O}_{3}\left(\mathrm{M}^{+}\right)=344.1177$. Found: 344.1173 .

3-(2-Chloro-acetylamino)-4-fluoro- $N$-(2-morpholin-4-yl-ethyl)-benzamide (12b)

3-Amino- $N$-(2-morpholin-4-yl-ethyl)-4-fluoro-benzamide $11 \mathrm{~b}(400 \mathrm{mg} ; 1.5 \mathrm{mmol})$ and 2-chloro-acetylchloride $(0.12 \mathrm{~mL} ; 1.5 \mathrm{mmol})$, to afford $\mathbf{1 2 b}(482 \mathrm{mg} ; 94 \%$ yield) as a white solid. m.p.: $129.1-130.8^{\circ} \mathrm{C}^{1}{ }^{1} \mathrm{H}-\mathrm{NMR}\left(\mathrm{CDCl}_{3}\right): \delta 2.48\left(\mathrm{t}, 4 \mathrm{H}, \mathrm{H}-4^{\prime}, J=4.4 \mathrm{~Hz}\right), 2.57\left(\mathrm{t}, 2 \mathrm{H}, \mathrm{H}-3^{\prime}, J=6.1 \mathrm{~Hz}\right)$, $3.50\left(\mathrm{q}, 2 \mathrm{H}, \mathrm{H}-2^{\prime}, J=5.6 \mathrm{~Hz}\right), 3.71\left(\mathrm{t}, 4 \mathrm{H}, \mathrm{H}-5^{\prime}, J=4.7 \mathrm{~Hz}\right), 4.20\left(\mathrm{~s}, 2 \mathrm{H}, \mathrm{H}-1^{\prime \prime}\right), 6.89$ (br. s, $\left.1 \mathrm{H}, \mathrm{H}-1^{\prime}\right)$, 7.14-7.19 (m, 1H, H-5), 7.64-7.68 (m, 1H, H-2), 8.57 (br. s, 1H, H-3), and 8.67 (dd, 1H, H-6, Jo = $7.3 \mathrm{~Hz}$, $\left.J_{m}=2 \mathrm{~Hz}\right)$ ppm. ${ }^{13} \mathrm{C}-\mathrm{NMR}\left(\mathrm{CDCl}_{3}\right): \delta 36.2,42.9,53.3(2 \mathrm{X}), 56.6,66.9(2 \mathrm{X}), 115.4\left(\mathrm{~d},{ }^{2} J_{\mathrm{C}-\mathrm{F}}=19.8 \mathrm{~Hz}\right)$, $119.5\left(\mathrm{~d},{ }^{4} J_{\mathrm{C}-\mathrm{F}}=1.5 \mathrm{~Hz}\right), 125.2\left(\mathrm{~d},{ }^{3} J_{\mathrm{C}-\mathrm{F}}=8.8 \mathrm{~Hz}\right), 125.4\left(\mathrm{~d},{ }^{2} J_{\mathrm{C}-\mathrm{F}}=11 \mathrm{~Hz}\right), 131.4\left(\mathrm{~d},{ }^{3} J_{\mathrm{C}-\mathrm{F}}=2.9 \mathrm{~Hz}\right)$, $154.3\left(\mathrm{~d},{ }^{1} J_{\mathrm{C}-\mathrm{F}}=250 \mathrm{~Hz}\right), 164.1$, and 166 ppm. HRMS: (EI) Calculated for $\mathrm{C}_{15} \mathrm{H}_{19} \mathrm{ClFN}_{3} \mathrm{O}_{3}\left(\mathrm{M}^{+}\right)=$ 344.1177. Found: 344.1176.

2-(2-Chloro-acetylamino)-5-fluoro- $\mathrm{N}$-(2-morpholin-4-yl-ethyl)-benzamide (12c)

2-Amino- $N$-(2-morpholin-4-yl-ethyl)-5-fluoro-benzamide 11c $(400 \mathrm{mg} ; 1.5 \mathrm{mmol})$ and 2-chloro-acetylchloride $(0.12 \mathrm{~mL} ; 1.5 \mathrm{mmol})$, to afford $12 \mathrm{c}(496 \mathrm{mg}, 97 \%$ yield) as a white solid. m.p.: 98.5-99.8 ${ }^{\circ} \mathrm{C}^{1}{ }^{1} \mathrm{H}-\mathrm{NMR}\left(\mathrm{CDCl}_{3}\right): \delta 2.55\left(\mathrm{t}, 4 \mathrm{H}, \mathrm{H}-4^{\prime}, J=4.4 \mathrm{~Hz}\right), 2.65\left(\mathrm{t}, 2 \mathrm{H}, \mathrm{H}-3^{\prime}, J=6.1 \mathrm{~Hz}\right), 3.57$

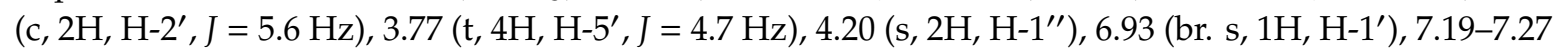
$(\mathrm{m}, 2 \mathrm{H}, \mathrm{H}-4$ and $\mathrm{H}-6), 8.61\left(\mathrm{dd}, 1 \mathrm{H}, \mathrm{H}-3, J_{o}=8.9 \mathrm{~Hz}, J_{m}=5.1 \mathrm{~Hz}\right.$ ), and 11.8 (br. s, 1H, H-2) ppm. 
${ }^{13} \mathrm{C}-\mathrm{NMR}\left(\mathrm{CDCl}_{3}\right): \delta 36.1,43.1,53.3(2 \mathrm{X}), 56.5,66.9(2 \mathrm{X}), 113.3\left(\mathrm{~d},{ }^{2} J_{\mathrm{C}-\mathrm{F}}=24 \mathrm{~Hz}\right), 119.2\left(\mathrm{~d},{ }^{4} J_{\mathrm{C}-\mathrm{F}}=22 \mathrm{~Hz}\right)$, $122.7\left(\mathrm{~d},{ }^{3} J_{\mathrm{C}-\mathrm{F}}=5.9 \mathrm{~Hz}\right), 123.6\left(\mathrm{~d},{ }^{2} J_{\mathrm{C}-\mathrm{F}}=7.3 \mathrm{~Hz}\right), 134.8\left(\mathrm{~d},{ }^{3} J_{\mathrm{C}-\mathrm{F}}=2.9 \mathrm{~Hz}\right), 158.2\left(\mathrm{~d},{ }^{1} J_{\mathrm{C}-\mathrm{F}}=245 \mathrm{~Hz}\right)$, 165.1, and 167.3 ppm. HRMS: (EI) Calculated for $\mathrm{C}_{15} \mathrm{H}_{19} \mathrm{ClFN}_{3} \mathrm{O}_{3}\left(\mathrm{M}^{+}\right)=344.1177$. Found: 344.1164.

5-(2-Chloro-acetylamino)-2-fluoro- $N$-(2-morpholin-4-yl-ethyl)-benzamide (12d)

5-Amino- $N$-(2-morpholin-4-yl-ethyl)-2-fluoro-benzamide 11d (400 mg; $1.5 \mathrm{mmol})$ and 2-chloro-acetylchloride (0.12 mL; $1.5 \mathrm{mmol})$, to afford $12 \mathrm{~d}$ (483 $\mathrm{mg} ; 94 \%$ yield) as a white solid. m.p.: $144.1-145.1^{\circ} \mathrm{C} ;{ }^{1} \mathrm{H}-\mathrm{NMR}\left(\mathrm{CDCl}_{3}\right): \delta 2.48\left(\mathrm{t}, 4 \mathrm{H}, \mathrm{H}-4^{\prime}, J=4.2 \mathrm{~Hz}\right), 2.57\left(\mathrm{t}, 2 \mathrm{H}, \mathrm{H}-3^{\prime}, J=5.9 \mathrm{~Hz}\right)$, $3.54\left(\mathrm{q}, 2 \mathrm{H}, \mathrm{H}-2^{\prime}, J=5.4 \mathrm{~Hz}\right), 3.69\left(\mathrm{t}, 4 \mathrm{H}, \mathrm{H}-5^{\prime}, J=4.4 \mathrm{~Hz}\right), 4.15\left(\mathrm{~s}, 2 \mathrm{H}, \mathrm{H}-\mathrm{-}^{\prime \prime}\right), 7.05-7.13(\mathrm{~m}, 1 \mathrm{H}, \mathrm{H}-3)$, 7.47 (br. s, $\left.1 \mathrm{H}, \mathrm{H}-1^{\prime}\right), 7.9$ (dd, $\left.1 \mathrm{H}, \mathrm{H}-4, J_{o}=6.5 \mathrm{~Hz}, J_{m}=2.7 \mathrm{~Hz}\right), 8.03-8.09(\mathrm{~m}, 1 \mathrm{H}, \mathrm{H}-6)$, and 8.70 (br. s, 1H, H-5) ppm. ${ }^{13} \mathrm{C}-\mathrm{NMR}\left(\mathrm{CDCl}_{3}\right): \delta 36.4,42.9,53.2(2 \mathrm{X}), 56.3,67(2 \mathrm{X}), 116.9\left(\mathrm{~d},{ }^{2} J_{\mathrm{C}-\mathrm{F}}=26.4 \mathrm{~Hz}\right), 121.5$ $\left(\mathrm{d},{ }^{\prime} J_{\mathrm{C}-\mathrm{F}}=13.2 \mathrm{~Hz}\right), 123.1\left(\mathrm{~d},{ }^{4} J_{\mathrm{C}-\mathrm{F}}=2.2 \mathrm{~Hz}\right), 125.2\left(\mathrm{~d},{ }^{3} J_{\mathrm{C}-\mathrm{F}}=8.8 \mathrm{~Hz}\right), 133.9\left(\mathrm{~d},{ }^{3^{\prime}} J_{\mathrm{C}-\mathrm{F}}=2.9 \mathrm{~Hz}\right), 157.4$ $\left(\mathrm{d},{ }^{1} J_{\mathrm{C}-\mathrm{F}}=246 \mathrm{~Hz}\right), 162.6$, and $164.4 \mathrm{ppm}$. HRMS: (EI) Calculated for $\mathrm{C}_{15} \mathrm{H}_{19} \mathrm{ClFN}_{3} \mathrm{O}_{3}\left(\mathrm{M}^{+}\right)=344.1177$. Found: 344.1173 .

3.1.8. General Procedure for the Synthesis of (2-\{4-[3-(1H-3-indolyl)-propyl]-1-piperazinyl\}acetylamino)- $N$-(2-morpholin-4-yl-ethyl)-fluorinated Benzamides Derivatives 13a-1

4-Fluoro-2-(2-\{4-[3-(5-fluor-1H-3-indolyl)-propyl]-1-piperazinyl\}-acetylamino)-N-(2-morpholin-4-ylethyl) Benzamide (13a) as a Model

To a stirred solution of 5-fluoro-3-(3-piperazin-1-yl-propyl)- $1 H$-indole $9 \mathbf{b}$ (152 $\mathrm{mg} ; 0.58 \mathrm{mmol})$, in dry $\mathrm{CH}_{3} \mathrm{CN}$ (50 mL), 2-(2-chloro-acetylamino)-4-fluoro- $N$-(2-morpholin-4-yl-ethyl)-benzamide 12a (199 mg; $0.58 \mathrm{mmol})$ and anhydrous $\mathrm{K}_{2} \mathrm{CO}_{3}(80 \mathrm{mg} ; 0.58 \mathrm{mmol})$ were added. The mixture was heated at $80{ }^{\circ} \mathrm{C}$ for $24 \mathrm{~h}$. After this time, the resulting mixture was poured into water $(100 \mathrm{~mL})$, extracted with EtOAc $(4 \times 50 \mathrm{~mL})$, dried over anhydrous $\mathrm{Na}_{2} \mathrm{SO}_{4}$, and concentrated under reduced pressure. The organic crude was purified by column chromatography EtOAc/MeOH (6:1) to give 13a (298 mg; 62.6\%) as a yellow light solid. m.p.: 91.3-93.1 ${ }^{\circ} \mathrm{C} ;{ }^{1} \mathrm{H}-\mathrm{NMR}\left(\mathrm{DMSO}-d_{6}\right): \delta 1.78\left(\mathrm{q}, 2 \mathrm{H}, \mathrm{H}-2^{\prime}\right.$, $J=7.4 \mathrm{~Hz}), 2.26-2.56\left(\mathrm{~m}, 16 \mathrm{H}, \mathrm{H}-3^{\prime}, \mathrm{H}-4^{\prime}, \mathrm{H}-5^{\prime}, \mathrm{H}-9^{\prime \prime}\right.$ and $\left.\mathrm{H}-1^{\prime \prime \prime}\right), 2.66\left(\mathrm{t}, 2 \mathrm{H}, \mathrm{H}-1^{\prime}, J=7.3 \mathrm{~Hz}\right), 3.13$ (s, 2H, H-6'), 3.45-3.62 (m, 6H, H-8', and H-2'"'), $6.90\left(\mathrm{td}, 1 \mathrm{H}, \mathrm{H}-6, J_{o}=9.2 \mathrm{~Hz}, J_{m}=2.5 \mathrm{~Hz}\right), 7.01$ $\left(\mathrm{td}, 1 \mathrm{H}, \mathrm{H}-5^{\prime \prime}, J_{o}=8.4 \mathrm{~Hz}, J_{m}=2.7 \mathrm{~Hz}\right), 7.20(\mathrm{~d}, 1 \mathrm{H}, \mathrm{H}-2, J=2.1 \mathrm{~Hz}), 7.25\left(\mathrm{dd}, 1 \mathrm{H}, \mathrm{H}-7, J_{o}=10.2 \mathrm{~Hz}\right.$, $\left.J_{m}=2.5 \mathrm{~Hz}\right), 7.32\left(\mathrm{dd}, 1 \mathrm{H}, \mathrm{H}-4, J_{o}=8.8 \mathrm{~Hz}, J_{m}=4.7 \mathrm{~Hz}\right), 7.68-7.76\left(\mathrm{~m}, 1 \mathrm{H}, \mathrm{H}-3^{\prime \prime}\right), 8.40\left(\mathrm{dd}, 1 \mathrm{H}, \mathrm{H}-6^{\prime \prime}\right.$, $\left.J_{o}=12.3 \mathrm{~Hz}, J_{m}=2.6 \mathrm{~Hz}\right), 8.62\left(\mathrm{t}, 1 \mathrm{H}, \mathrm{H}-7^{\prime \prime}, J=5.5 \mathrm{~Hz}\right), 10.87(\mathrm{~s}, 1 \mathrm{H}, \mathrm{H}-1)$, and $12.24\left(\mathrm{br} . \mathrm{s}, 1 \mathrm{H}, \mathrm{H}-7^{\prime}\right)$ ppm. ${ }^{13} \mathrm{C}-\mathrm{NMR}$ (DMSO- $d_{6}$ ): $\delta 22.2,27.0,36.4,52.4(2 \mathrm{X}), 53.0(2 \mathrm{X}), 53.2(2 \mathrm{X}), 57.1,57.5,61.8,66.1(2 \mathrm{X})$, $102.7\left(\mathrm{~d},{ }^{2} J_{\mathrm{C}-\mathrm{F}}=23.1 \mathrm{~Hz}\right), 106.5\left(\mathrm{~d},{ }^{2} J_{\mathrm{C}-\mathrm{F}}=28.2 \mathrm{~Hz}\right), 108.8\left(\mathrm{~d},{ }^{2 \prime \prime} J_{\mathrm{C}-\mathrm{F}}=26 \mathrm{~Hz}\right), 109.1\left(\mathrm{~d},{ }^{2 \prime \prime \prime} J_{\mathrm{C}-\mathrm{F}}=22.3\right.$ $\mathrm{Hz}), 112.1\left(\mathrm{~d},{ }^{3} J_{\mathrm{C}-\mathrm{F}}=9.5 \mathrm{~Hz}\right) 114.7\left(\mathrm{~d},{ }^{4} J_{\mathrm{C}-\mathrm{F}}=4.8 \mathrm{~Hz}\right), 118.2\left(\mathrm{~d},{ }^{4} J_{\mathrm{C}-\mathrm{F}}=2.9 \mathrm{~Hz}\right), 124.3,127.4\left(\mathrm{~d},{ }^{3} J_{\mathrm{C}-\mathrm{F}}=\right.$ $9.5 \mathrm{~Hz}), 130.2\left(\mathrm{~d}, 3^{3 \prime \prime} J_{\mathrm{C}-\mathrm{F}}=9.9 \mathrm{~Hz}\right), 132.9,140.2\left(\mathrm{~d}, 3^{3^{\prime \prime \prime}} J_{\mathrm{C}-\mathrm{F}}=12.1 \mathrm{~Hz}\right), 156.5\left(\mathrm{~d},{ }^{1} J_{\mathrm{C}-\mathrm{F}}=231 \mathrm{~Hz}\right), 163.3$ $\left(\mathrm{d},{ }^{1} J_{\mathrm{C}-\mathrm{F}}=246 \mathrm{~Hz}\right), 166.8$, and 169.8 ppm. HRMS: (EI) Calculated for $\mathrm{C}_{30} \mathrm{H}_{38} \mathrm{~F}_{2} \mathrm{~N}_{6} \mathrm{O}_{3}\left(\mathrm{M}^{+}\right)=569.3051$. Found: 569.3050 .

4-Fluoro-3-(2-\{4-[3-(5-fluor-1H-3-indolyl)-propyl]-1-piperazinyl\}-acetylamino)-N-(2-morpholin-4yl-ethyl) Benzamide (13b)

5-Fluoro-3-(3-piperazin-1-yl-propyl)-1H-indole $9 \mathrm{~b}$ (152 mg; $0.58 \mathrm{mmol})$, 3-(2-chloro-acetylamino)4-fluor- $\mathrm{N}$-(2-morpholin-4-yl-ethyl)-benzamide $12 \mathbf{b}$ (199 mg; $0.58 \mathrm{mmol}$ ), and anhydrous $\mathrm{K}_{2} \mathrm{CO}_{3}(80 \mathrm{mg}$; $0.58 \mathrm{mmol}$ ), to afford $\mathbf{1 3 b}$ ( $150 \mathrm{mg} ; 47 \%$ yield) as a yellow light solid. m.p.: $92.2-93.9{ }^{\circ} \mathrm{C} ;{ }^{1} \mathrm{H}-\mathrm{NMR}$ (DMSO- $d_{6}$ ): $\delta 1.78\left(\mathrm{q}, 2 \mathrm{H}, \mathrm{H}-2^{\prime}, J=7.4 \mathrm{~Hz}\right), 2.27-2.56\left(\mathrm{~m}, 16 \mathrm{H}, \mathrm{H}-3^{\prime}, \mathrm{H}-4^{\prime}, \mathrm{H}-5^{\prime}, \mathrm{H}-9^{\prime \prime}\right.$ and $\left.\mathrm{H}-1^{\prime \prime \prime}\right), 2.66$ $\left(\mathrm{t}, 2 \mathrm{H}, \mathrm{H}-1^{\prime}, J=7.3 \mathrm{~Hz}\right), 3.19\left(\mathrm{~s}, 2 \mathrm{H}, \mathrm{H}-6^{\prime}\right), 3.33-3.36\left(\mathrm{~m}, 2 \mathrm{H}, \mathrm{H}-8^{\prime}\right), 3.57\left(\mathrm{t}, 4 \mathrm{H}, \mathrm{H}-2^{\prime \prime \prime}, J=4.4 \mathrm{~Hz}\right), 6.89$ $\left(\mathrm{td}, 1 \mathrm{H}, \mathrm{H}-6, J_{o}=9.2 \mathrm{~Hz}, J_{m}=2.5 \mathrm{~Hz}\right), 7.20(\mathrm{~d}, 1 \mathrm{H}, \mathrm{H}-2, J=1.9 \mathrm{~Hz}), 7.26\left(\mathrm{dd}, 1 \mathrm{H}, \mathrm{H}-7, J_{o}=10.1 \mathrm{~Hz}\right.$, $\left.J_{m}=2.4 \mathrm{~Hz}\right), 7.29-7.42(\mathrm{~m}, 2 \mathrm{H}, \mathrm{H}-4$ and H-5" $), 7.59-7.67\left(\mathrm{~m}, 1 \mathrm{H}, \mathrm{H}-2^{\prime \prime}\right), 8.40-8.50\left(\mathrm{~m}, 2 \mathrm{H}, \mathrm{H}-6^{\prime \prime}\right.$, and H-7"), 9.68 (br. s, $1 \mathrm{H}, \mathrm{H}-7^{\prime}$ ), and 10.87 (s, $1 \mathrm{H}, \mathrm{H}-1$ ) ppm. ${ }^{13} \mathrm{C}-\mathrm{NMR}$ (DMSO- $d_{6}$ ): $\delta 22.7,27.5,37.1,53.3$ (2X), $53.4(2 X), 53.7(2 X), 57.7,57.9,61.6,66.7(2 X), 103.4\left(\mathrm{~d},{ }^{2} J_{\mathrm{C}-\mathrm{F}}=23.5 \mathrm{~Hz}\right), 109.3\left(\mathrm{~d},{ }^{2}{ }^{\prime \prime} J_{\mathrm{C}-\mathrm{F}}=26.4 \mathrm{~Hz}\right)$, $112.6\left(\mathrm{~d},{ }^{3^{\prime}} J_{\mathrm{C}-\mathrm{F}}=9.5 \mathrm{~Hz}\right), 115.2\left(\mathrm{~d},{ }^{4} J_{\mathrm{C}-\mathrm{F}}=4.4 \mathrm{~Hz}\right), 115.6\left(\mathrm{~d},{ }^{2^{\prime}} J_{\mathrm{C}-\mathrm{F}}=19.8 \mathrm{~Hz}\right), 123\left(\mathrm{~d},{ }^{4} J_{\mathrm{C}-\mathrm{F}}=6.6 \mathrm{~Hz}\right)$, 
$124.5\left(\mathrm{~d},{ }^{3} J_{\mathrm{C}-\mathrm{F}}=8.1 \mathrm{~Hz}\right), 124.9,126.2\left(\mathrm{~d},{ }^{2}{ }^{\prime \prime \prime} J_{\mathrm{C}-\mathrm{F}}=11.7 \mathrm{~Hz}\right), 127.9\left(\mathrm{~d},{ }^{3} J_{\mathrm{C}-\mathrm{F}}=9.5 \mathrm{~Hz}\right), 131.6\left(\mathrm{~d},{ }^{3} J_{\mathrm{C}-\mathrm{F}}=\right.$ $2.9 \mathrm{~Hz}), 133.4,155.3\left(\mathrm{~d},{ }^{1} J_{\mathrm{C}-\mathrm{F}}=248 \mathrm{~Hz}\right), 157.0\left(\mathrm{~d},{ }^{1} J_{\mathrm{C}-\mathrm{F}}=230.4 \mathrm{~Hz}\right) .165 .6$, and $169.1 \mathrm{ppm}$. HRMS: (EI) Calculated for $\mathrm{C}_{30} \mathrm{H}_{38} \mathrm{~F}_{2} \mathrm{~N}_{6} \mathrm{O}_{3}\left(\mathrm{M}^{+}\right)=569.3051$. Found: 569.3046 .

2-Fluoro-5-(2-\{4-[3-(5-fluor-1H-3-indolyl)-propyl]-1-piperazinyl\}-acetylamino)-N-(2-morpholin-4-ylethyl) Benzamide (13c)

5-Fluoro-3-(3-piperazin-1-yl-propyl)- $1 H$-indole $9 \mathrm{~b}$ (152 mg; $0.58 \mathrm{mmol})$, 5-(2-Chloro-acetylamino)2-fluoro- $\mathrm{N}$-(2-morpholin-4-yl-ethyl)-benzamide 12d (199 mg; $0.58 \mathrm{mmol})$, and anhydrous $\mathrm{K}_{2} \mathrm{CO}_{3}$ (80 mg; $0.58 \mathrm{mmol})$, to afford $13 \mathrm{c}\left(178 \mathrm{mg}\right.$; $55 \%$ yield) as a yellow light solid. m.p.: $91.8-93.2{ }^{\circ} \mathrm{C}$; ${ }^{1} \mathrm{H}-\mathrm{NMR}\left(\mathrm{DMSO}-d_{6}\right): \delta 1.76\left(\mathrm{q}, 2 \mathrm{H}, \mathrm{H}-2^{\prime}, J=7.4 \mathrm{~Hz}\right), 2.32\left(\mathrm{t}, 2 \mathrm{H}, \mathrm{H}-3^{\prime}, J=6.9 \mathrm{~Hz}\right), 2.36-2.56(\mathrm{~m}, 14 \mathrm{H}$, $\mathrm{H}-4^{\prime}, \mathrm{H}-5^{\prime}, \mathrm{H}-9^{\prime \prime}$ and $\left.\mathrm{H}-\mathrm{1}^{\prime \prime \prime}\right), 2.65\left(\mathrm{t}, 2 \mathrm{H}, \mathrm{H}-1^{\prime}, J=7.3 \mathrm{~Hz}\right), 3.11\left(\mathrm{~s}, 2 \mathrm{H}, \mathrm{H}-6^{\prime}\right), 3.36-3.42\left(\mathrm{~m}, 2 \mathrm{H}, \mathrm{H}-8^{\prime \prime}\right)$, $3.57\left(\mathrm{t}, 4 \mathrm{H}, \mathrm{H}-2^{\prime \prime \prime}, J=4.5 \mathrm{~Hz}\right), 6.89\left(\mathrm{td}, 1 \mathrm{H}, \mathrm{H}-6, J_{o}=8.2 \mathrm{~Hz}, J_{m}=2.6 \mathrm{~Hz}\right), 7.17-7.28(\mathrm{~m}, 3 \mathrm{H}, \mathrm{H}-2, \mathrm{H}-7$ and $\left.\mathrm{H}-3^{\prime \prime}\right), 7.31\left(\mathrm{dd}, 1 \mathrm{H}, \mathrm{H}-4, J_{o}=8.8 \mathrm{~Hz}, J_{m}=4.6 \mathrm{~Hz}\right), 7.73-7.81\left(\mathrm{~m}, 1 \mathrm{H}, \mathrm{H}-6^{\prime \prime}\right), 7.92\left(\mathrm{dd}, 1 \mathrm{H}, \mathrm{H}-4^{\prime \prime}\right.$, $\left.J_{o}=6.5 \mathrm{~Hz}, J_{m}=2.8 \mathrm{~Hz}\right), 8.13-8.24\left(\mathrm{~m}, 1 \mathrm{H}, \mathrm{H}-7^{\prime \prime}\right), 9.90\left(\right.$ br. s, $\left.1 \mathrm{H}, \mathrm{H}-7^{\prime}\right)$, and $10.89(\mathrm{~s}, 1 \mathrm{H}, \mathrm{H}-1) \mathrm{ppm}$. ${ }^{13} \mathrm{C}-\mathrm{NMR}\left(\mathrm{DMSO}-d_{6}\right): \delta 22.7,27.6,37,53.1(2 \mathrm{X}), 53.4(2 \mathrm{X}), 53.7(2 \mathrm{X}), 57.4,57.9,62.3,66.7(2 \mathrm{X}), 103.4(\mathrm{~d}$, $\left.{ }^{2} J_{\mathrm{C}-\mathrm{F}}=22.7 \mathrm{~Hz}\right), 109.3\left(\mathrm{~d},{ }^{2} J_{\mathrm{C}-\mathrm{F}}=26.5 \mathrm{~Hz}\right), 112.6\left(\mathrm{~d},{ }^{3{ }^{\prime \prime}} J_{\mathrm{C}-\mathrm{F}}=9.9 \mathrm{~Hz}\right), 115.2\left(\mathrm{~d},{ }^{4} J_{\mathrm{C}-\mathrm{F}}=4.4 \mathrm{~Hz}\right), 116.7$ $\left(\mathrm{d},{ }^{2 \prime \prime} J_{\mathrm{C}-\mathrm{F}}=23.8 \mathrm{~Hz}\right), 121.33\left(\mathrm{~d},{ }^{4} J_{\mathrm{C}-\mathrm{F}}=2.2 \mathrm{~Hz}\right), 123.6\left(\mathrm{~d},{ }^{2 \prime \prime \prime} J_{\mathrm{C}-\mathrm{F}}=8.3 \mathrm{~Hz}\right), 124.2\left(\mathrm{~d},{ }^{3} J_{\mathrm{C}-\mathrm{F}}=9.5 \mathrm{~Hz}\right)$, $124.9,127.9\left(\mathrm{~d}, 3^{\prime \prime \prime \prime} J_{\mathrm{C}-\mathrm{F}}=9.4 \mathrm{~Hz}\right), 133.6,135.5\left(\mathrm{~d},{ }^{\prime} J_{\mathrm{C}-\mathrm{F}}=3.3 \mathrm{~Hz}\right), 155.4\left(\mathrm{~d},{ }^{1} J_{\mathrm{C}-\mathrm{F}}=245 \mathrm{~Hz}\right), 157(\mathrm{~d}$, $\left.{ }^{\prime} J_{\mathrm{C}-\mathrm{F}}=231 \mathrm{~Hz}\right), 163.7$, and 168.9 ppm. HRMS: (EI) Calculated for $\mathrm{C}_{30} \mathrm{H}_{38} \mathrm{~F}_{2} \mathrm{~N}_{6} \mathrm{O}_{3}\left(\mathrm{M}^{+}\right)=569.3051$. Found: 569.3047.

5-Fluoro-2-(2-\{4-[3-(5-fluoro-1H-3-indolyl)-propyl]-1-piperazinyl\}-acetylamino)- $N$-(2-morpholin-4-ylethyl) Benzamide (13d)

5-Fluoro-3-(3-piperazin-1-yl-propyl)-1H-indole $\quad 9 b \quad(152 \quad \mathrm{mg} ; \quad 0.58 \quad \mathrm{mmol}), \quad 2$-(2-chloroacetylamino)-5-fluoro- $\mathrm{N}$-(2-morpholin-4-yl-ethyl)-benzamide $12 \mathrm{c}(199 \mathrm{mg} ; 0.58 \mathrm{mmol})$, and anhydrous $\mathrm{K}_{2} \mathrm{CO}_{3}(80 \mathrm{mg} ; 0.58 \mathrm{mmol})$, to afford $13 \mathrm{~d}$ (188 mg; 59\% yield) as a yellow light solid. m.p.: $94.2-95.4$ ${ }^{\circ} \mathrm{C} ;{ }^{1} \mathrm{H}-\mathrm{NMR}$ (DMSO- $d_{6}$ ): $\delta 1.79$ (q, 2H, H-2',$\left.J=7.2 \mathrm{~Hz}\right), 2.32-2.51$ (m, 16H, H-3', H-4', H-5' , H-9' , and $\left.\mathrm{H}-1^{\prime \prime \prime}\right), 2.66\left(\mathrm{t}, 2 \mathrm{H}, \mathrm{H}-1^{\prime}, J=7.3 \mathrm{~Hz}\right), 3.10\left(\mathrm{~s}, 2 \mathrm{H}, \mathrm{H}-6^{\prime}\right), 3.50-3.60\left(\mathrm{~m}, 6 \mathrm{H}, \mathrm{H}-8^{\prime \prime}\right.$, and $\left.\mathrm{H}-2^{\prime \prime \prime}\right), 6.89$ (td, $1 \mathrm{H}$, $\left.\mathrm{H}-6, J_{o}=9.2 \mathrm{~Hz}, J_{m}=2.5 \mathrm{~Hz}\right), 7.20(\mathrm{~d}, 1 \mathrm{H}, \mathrm{H}-2, J=2.0 \mathrm{~Hz}), 7.25\left(\mathrm{dd}, 1 \mathrm{H}, \mathrm{H}-7, J_{o}=10.1 \mathrm{~Hz}, J_{m}=2.5\right.$ $\mathrm{Hz}), 7.29-7.40\left(\mathrm{~m}, 2 \mathrm{H}, \mathrm{H}-4\right.$ and H-4" $\left.{ }^{\prime \prime}\right), 7.49\left(\mathrm{dd}, 1 \mathrm{H}, \mathrm{H}-3^{\prime \prime}, J_{o}=9.5 \mathrm{~Hz}, J_{m}=3.0 \mathrm{~Hz}\right), 8.53\left(\mathrm{dd}, 1 \mathrm{H}, \mathrm{H}-6^{\prime \prime}\right.$, $\left.J_{o}=9.2 \mathrm{~Hz}, J_{m}=5.4 \mathrm{~Hz}\right), 8.69\left(\mathrm{t}, 1 \mathrm{H}, \mathrm{H}-7^{\prime \prime}, J=5.4 \mathrm{~Hz}\right), 10.89(\mathrm{~s}, 1 \mathrm{H}, \mathrm{H}-1)$, and $11.62\left(\right.$ br. s, $\left.1 \mathrm{H}, \mathrm{H}-7^{\prime}\right)$ ppm. ${ }^{13} \mathrm{C}-\mathrm{NMR}$ (DMSO- $d_{6}$ ): $\delta$ 22.2, 26.9, 36.4, $52.4(2 \mathrm{X}), 52.9(2 \mathrm{X}), 53.2(2 \mathrm{X}), 57,57.4,61.7,66.1(2 \mathrm{X})$, $102.9\left(\mathrm{~d},{ }^{2} J_{\mathrm{C}-\mathrm{F}}=22.3 \mathrm{~Hz}\right), 108.8\left(\mathrm{~d},{ }^{2} J_{\mathrm{C}-\mathrm{F}}=26.3 \mathrm{~Hz}\right), 112.1\left(\mathrm{~d},{ }^{3} J_{\mathrm{C}-\mathrm{F}}=9.9 \mathrm{~Hz}\right), 114.5\left(\mathrm{~d},{ }^{2{ }^{\prime \prime}} J_{\mathrm{C}-\mathrm{F}}=23.8 \mathrm{~Hz}\right)$, $114.6\left(\mathrm{~d},{ }^{4} J_{\mathrm{C}-\mathrm{F}}=5 \mathrm{~Hz}\right), 118\left(\mathrm{~d},{ }^{2 \prime \prime \prime} J_{\mathrm{C}-\mathrm{F}}=22 \mathrm{~Hz}\right), 122.1\left(\mathrm{~d},{ }^{3} J_{\mathrm{C}-\mathrm{F}}=7 \mathrm{~Hz}\right), 123.8\left(\mathrm{~d},{ }^{3 \prime \prime} J_{\mathrm{C}-\mathrm{F}}=6.2 \mathrm{~Hz}\right), 124.3$, $127.4\left(\mathrm{~d},{ }^{3^{\prime \prime \prime}} J_{\mathrm{C}-\mathrm{F}}=9.5 \mathrm{~Hz}\right), 132.9,134.4\left(\mathrm{~d},{ }^{4} J_{\mathrm{C}-\mathrm{F}}=2.2 \mathrm{~Hz}\right), 152.4\left(\mathrm{~d},{ }^{1} J_{\mathrm{C}-\mathrm{F}}=231 \mathrm{~Hz}\right), 156.5\left(\mathrm{~d},{ }^{1} J_{\mathrm{C}-\mathrm{F}}\right.$ $=223 \mathrm{~Hz}$ ), 166.3, and 169 ppm. HRMS: (EI) Calculated for $\mathrm{C}_{30} \mathrm{H}_{38} \mathrm{~F}_{2} \mathrm{~N}_{6} \mathrm{O}_{3}\left(\mathrm{M}^{+}\right)=569.3051$. Found: 569.3048 .

4-Fluoro-2-(2-\{4-[3-(5-bromo-1H-3-indolyl)-propyl]-1-piperazinyl\}-acetylamino)- $N$-(2-morpholin-4-ylethyl) Benzamide (13e)

5-Bromo-3-(3-piperazin-1-yl-propyl)-1H-indole 9c (187 mg; $0.58 \mathrm{mmol})$, 2-(2-chloro-acetylamino)-4fluoro- $\mathrm{N}$-(2-morpholin-4-yl-ethyl)-benzamide $12 \mathrm{a}(199 \mathrm{mg}$; $0.58 \mathrm{mmol})$, and anhydrous $\mathrm{K}_{2} \mathrm{CO}_{3}(80 \mathrm{mg}$; $0.58 \mathrm{mmol})$, to afford $13 \mathrm{e}\left(236 \mathrm{mg} ; 66 \%\right.$ yield) as a yellow light solid. m.p.: $92.1-93.8^{\circ} \mathrm{C} ;{ }^{1} \mathrm{H}-\mathrm{NMR}$ (DMSO- $\left.d_{6}\right): \delta 1.78$ (q, 2H, H-2',$\left.J=7.4 \mathrm{~Hz}\right), 2.35\left(\mathrm{t}, 2 \mathrm{H}, \mathrm{H}-3^{\prime}, J=6.9 \mathrm{~Hz}\right), 2.38-2.52\left(\mathrm{~m}, 14 \mathrm{H}, \mathrm{H}-4^{\prime}, \mathrm{H}-5^{\prime}\right.$, $\mathrm{H}-9^{\prime \prime}$ and $\left.\mathrm{H}-1^{\prime \prime \prime}\right), 2.67\left(\mathrm{t}, 2 \mathrm{H}, \mathrm{H}-\mathrm{1}^{\prime}, J=7.3 \mathrm{~Hz}\right), 3.13\left(\mathrm{~s}, 2 \mathrm{H}, \mathrm{H}-6^{\prime}\right), 3.40-3.42\left(\mathrm{~m}, 2 \mathrm{H}, \mathrm{H}-8^{\prime \prime}\right), 3.54(\mathrm{t}, 4 \mathrm{H}$, $\left.\mathrm{H}-2^{\prime \prime \prime}, J=4.5 \mathrm{~Hz}\right), 7.0\left(\mathrm{td}, 1 \mathrm{H}, \mathrm{H}-5^{\prime \prime}, J_{o}=8.3 \mathrm{~Hz}, J_{m}=2.8 \mathrm{~Hz}\right), 7.12-7.20(\mathrm{~m}, 2 \mathrm{H}, \mathrm{H}-2$, and H-6), $7.31(\mathrm{~d}$, $1 \mathrm{H}, \mathrm{H}-7, J=8.6 \mathrm{~Hz}), 7.62(\mathrm{~d}, 1 \mathrm{H}, \mathrm{H}-4, J=1.7 \mathrm{~Hz}), 7.77\left(\mathrm{dd}, 1 \mathrm{H}, \mathrm{H}-3^{\prime \prime}, J_{o}=8.8 \mathrm{~Hz}, J_{m}=6.6 \mathrm{~Hz}\right), 8.39$ $\left(\mathrm{dd}, 1 \mathrm{H}, \mathrm{H}-6^{\prime \prime}, J_{o}=12.3 \mathrm{~Hz}, J_{m}=2.7 \mathrm{~Hz}\right), 8.70\left(\mathrm{t}, 1 \mathrm{H}, \mathrm{H}-7^{\prime \prime}, J=5.4 \mathrm{~Hz}\right), 11.1(\mathrm{~s}, 1 \mathrm{H}, \mathrm{H}-1)$, and 12.1 (br. s, $1 \mathrm{H}, \mathrm{H}-7^{\prime}$ ) ppm. ${ }^{13} \mathrm{C}-\mathrm{NMR}$ (DMSO- $\left.d_{6}\right): \delta 22.8,27.4,36.9,52.6(2 \mathrm{X}), 52.7(2 \mathrm{X}), 53.7(2 \mathrm{X}), 56.2,57.6$, $62.2,66.6(2 \mathrm{X}), 107.1\left(\mathrm{~d},{ }^{2} J_{\mathrm{C}-\mathrm{F}}=26.5 \mathrm{~Hz}\right), 109.7\left(\mathrm{~d},{ }^{2} J_{\mathrm{C}-\mathrm{F}}=22.7 \mathrm{~Hz}\right), 111.2,113.3,118.6\left(\mathrm{~d},{ }^{4} J_{\mathrm{C}-\mathrm{F}}=4.9\right.$ 
$\mathrm{Hz}), 118.7,121.6,123.7,124.4,129.6,130.8\left(\mathrm{~d},{ }^{3} J_{\mathrm{C}-\mathrm{F}}=9.9 \mathrm{~Hz}\right), 135.4,140.7\left(\mathrm{~d},{ }^{3} J_{\mathrm{C}-\mathrm{F}}=12.2 \mathrm{~Hz}\right), 163.8(\mathrm{~d}$, $\left.{ }^{1} J_{\mathrm{C}-\mathrm{F}}=246 \mathrm{~Hz}\right), 167.3$, and 170.4 ppm. HRMS: (EI) Calculated for $\mathrm{C}_{30} \mathrm{H}_{38} \mathrm{BrFN}_{6} \mathrm{O}_{3}\left(\mathrm{M}^{+}\right)=629.2251$. Found: 629.2246 .

4-Fluoro-3-(2-\{4-[3-(5-bromo-1H-3-indolyl)-propyl]-1-piperazinyl\}-acetylamino)- $N$-(2-morpholin-4-ylethyl) Benzamide (13f)

5-Bromo-3-(3-piperazin-1-yl-propyl)-1H-indole 9c (187 mg; $0.58 \mathrm{mmol})$, 3-(2-chloro-acetylamino)4-fluoro- $\mathrm{N}$-(2-morpholin-4-yl-ethyl)-benzamide $\mathbf{1 2 b}(199 \mathrm{mg} ; 0.58 \mathrm{mmol})$, and anhydrous $\mathrm{K}_{2} \mathrm{CO}_{3}$ ( $80 \mathrm{mg}$; $0.58 \mathrm{mmol}$ ), to afford $13 \mathrm{f}$ (193 mg; $54 \%$ yield) as a yellow light solid. m.p.: $85.2-86.8{ }^{\circ} \mathrm{C}$; ${ }^{1} \mathrm{H}-\mathrm{NMR}$ (DMSO- $\left.d_{6}\right): \delta 1.78\left(\mathrm{q}, 2 \mathrm{H}, \mathrm{H}-2^{\prime}, J=6.5 \mathrm{~Hz}\right), 2.30-2.55\left(\mathrm{~m}, 16 \mathrm{H}, \mathrm{H}-3^{\prime}, \mathrm{H}-4^{\prime}, \mathrm{H}-5^{\prime}, \mathrm{H}-9^{\prime \prime}\right.$, and $\left.\mathrm{H}-1^{\prime \prime \prime}\right), 2.68\left(\mathrm{t}, 2 \mathrm{H}, \mathrm{H}-1^{\prime}, J=7.3 \mathrm{~Hz}\right), 3.20\left(\mathrm{~s}, 2 \mathrm{H}, \mathrm{H}-6^{\prime}\right), 3.40-3.44\left(\mathrm{~m}, 2 \mathrm{H}, \mathrm{H}-8^{\prime \prime}\right), 3.57$ (t, 4H, H-2'"', $J=4.4 \mathrm{~Hz}), 7.13-7.21\left(\mathrm{~m}, 2 \mathrm{H}, \mathrm{H}-2\right.$, and H-6), $7.31(\mathrm{~d}, 1 \mathrm{H}, \mathrm{H}-7, J=10.1 \mathrm{~Hz}), 7.34-7.42\left(\mathrm{~m}, 1 \mathrm{H}, \mathrm{H}-5^{\prime \prime}\right)$, 7.59-7.67 (m, 1H, H-2" ) , $7.62(\mathrm{~d}, 1 \mathrm{H}, \mathrm{H}-4, J=1.6 \mathrm{~Hz}), 8.37-8.52\left(\mathrm{~m}, 2 \mathrm{H}, \mathrm{H}-6^{\prime \prime}\right.$ and H-7"), 9.69 (br. s, $1 \mathrm{H}$, H-7'), and $11.0(\mathrm{~s}, 1 \mathrm{H}, \mathrm{H}-1)$ ppm. ${ }^{13} \mathrm{C}-\mathrm{NMR}$ (DMSO- $\left.d_{6}\right): \delta 22.2,26.9,36.4,52.4(2 \mathrm{X}), 52.9(2 \mathrm{X}), 53.2(2 \mathrm{X})$, $57,57.4,61.7,66.1(2 \mathrm{X}), 111.3,113.8,114.7,115.6\left(\mathrm{~d},{ }^{2} J_{\mathrm{C}-\mathrm{F}}=21 \mathrm{~Hz}\right), 121.1,123.1,123.28\left(\mathrm{~d},{ }^{4} J_{\mathrm{C}-\mathrm{F}}=2.9 \mathrm{~Hz}\right)$, $123.7,124.5\left(\mathrm{~d},{ }^{3} J_{\mathrm{C}-\mathrm{F}}=9.5 \mathrm{~Hz}\right), 126.2\left(\mathrm{~d},{ }^{2} J_{\mathrm{C}-\mathrm{F}}=11 \mathrm{~Hz}\right), 129.7,131.63\left(\mathrm{~d},{ }^{3} J_{\mathrm{C}-\mathrm{F}}=3.7 \mathrm{~Hz}\right), 135.7,155.3$ $\left(\mathrm{d},{ }^{1} J_{\mathrm{C}-\mathrm{F}}=248 \mathrm{~Hz}\right), 165.6$, and 169 ppm. HRMS: (EI) Calculated for $\mathrm{C}_{30} \mathrm{H}_{38} \mathrm{BrFN}_{6} \mathrm{O}_{3}\left(\mathrm{M}^{+}\right)=629.2251$. Found: 629.2250 .

2-Fluoro-5-(2-\{4-[3-(5-bromo-1H-3-indolyl)-propyl]-1-piperazinyl\}-acetylamino)- $N$-(2-morpholin-4-ylethyl) Benzamide (13g)

5-Bromo-3-(3-piperazin-1-yl-propyl)-1 $H$-indole $9 \mathrm{c}$ (187 mg; $0.58 \mathrm{mmol})$, 5-(2-chloro-acetylamino)-2fluoro- $N$-(2-morpholin-4-yl-ethyl)-benzamide $12 \mathrm{~d}(199 \mathrm{mg} ; 0.58 \mathrm{mmol})$, and anhydrous $\mathrm{K}_{2} \mathrm{CO}_{3}(80$ $\mathrm{mg} ; 0.58 \mathrm{mmol})$, to afford $13 \mathrm{~g}$ ( $249 \mathrm{mg} ; 70 \%$ yield) as a yellow light solid. m.p.: $73.2-74.5^{\circ} \mathrm{C} ;{ }^{1} \mathrm{H}-\mathrm{NMR}$ (DMSO- $\left.d_{6}\right): \delta 1.79\left(\mathrm{q}, 2 \mathrm{H}, \mathrm{H}-2^{\prime}, J=7.2 \mathrm{~Hz}\right), 2.32-2.51\left(\mathrm{~m}, 16 \mathrm{H}, \mathrm{H}-3^{\prime}, \mathrm{H}-4^{\prime}, \mathrm{H}-5^{\prime}, \mathrm{H}-9^{\prime \prime}\right.$, and $\left.\mathrm{H}-1^{\prime \prime \prime}\right), 2.66$ $\left(\mathrm{t}, 2 \mathrm{H}, \mathrm{H}-1^{\prime}, J=7.3 \mathrm{~Hz}\right), 3.10$ (s, 2H, H-6'), 3.50-3.60 (m, 6H, H-8' , and H-2'"'), 6.89 (td, 1H, H-6, $J_{o}=9.2$ $\left.\mathrm{Hz}, J_{m}=2.5 \mathrm{~Hz}\right), 7.20(\mathrm{~d}, 1 \mathrm{H}, \mathrm{H}-2, J=2.0 \mathrm{~Hz}), 7.25\left(\mathrm{dd}, 1 \mathrm{H}, \mathrm{H}-7, J_{o}=10.1 \mathrm{~Hz}, J_{m}=2.5 \mathrm{~Hz}\right), 7.29-7.40$ $\left(\mathrm{m}, 2 \mathrm{H}, \mathrm{H}-4\right.$, and $\left.\mathrm{H6}^{\prime \prime}\right), 7.49\left(\mathrm{dd}, 1 \mathrm{H}, \mathrm{H}-4^{\prime \prime}, J_{o}=9.5 \mathrm{~Hz}, J_{m}=3.0 \mathrm{~Hz}\right), 8.53\left(\mathrm{dd}, 1 \mathrm{H}, \mathrm{H}-3^{\prime \prime}, J_{o}=9.2 \mathrm{~Hz}\right.$, $\left.J_{m}=5.4 \mathrm{~Hz}\right), 8.69\left(\mathrm{t}, 1 \mathrm{H}, \mathrm{H}-7^{\prime \prime}, J=5.4 \mathrm{~Hz}\right), 10.89(\mathrm{~s}, 1 \mathrm{H}, \mathrm{H}-1)$, and $11.62\left(\mathrm{br} . \mathrm{s}, 1 \mathrm{H}, \mathrm{H}-7^{\prime}\right)$ ppm. ${ }^{13} \mathrm{C}-\mathrm{NMR}$ (DMSO- $d_{6}$ ): $\delta 22.4,27.5,36.9,52.9$ (2X), 53 (2X), $53.6(2 X), 574,57.6,62.1,66.7$ (2X), 111.3, 113.8, 114.7, $116.7\left(\mathrm{~d},{ }^{2} J_{\mathrm{C}-\mathrm{F}}=23.5 \mathrm{~Hz}\right), 121.1,121.4\left(\mathrm{~d},{ }^{3} J_{\mathrm{C}-\mathrm{F}}=3.7 \mathrm{~Hz}\right), 123.6\left(\mathrm{~d},{ }^{3} J_{\mathrm{C}-\mathrm{F}}=7.3 \mathrm{~Hz}\right), 123.7,124.2\left(\mathrm{~d},{ }^{2} J_{\mathrm{C}-\mathrm{F}}\right.$ $=15.4 \mathrm{~Hz}), 124.5,129.6,135.3,135.4\left(\mathrm{~d},{ }^{4} J_{\mathrm{C}-\mathrm{F}}=2.2 \mathrm{~Hz}\right), 155.4\left(\mathrm{~d},{ }^{1} J_{\mathrm{C}-\mathrm{F}}=245 \mathrm{~Hz}\right), 163.7$, and $168.9 \mathrm{ppm}$. HRMS: (EI) Calculated for $\mathrm{C}_{30} \mathrm{H}_{38} \mathrm{BrFN}_{6} \mathrm{O}_{3}\left(\mathrm{M}^{+}\right)=629.2251$. Found: 629.2248.

5-Fluoro-2-(2-\{4-[3-(5-bromo-1H-3-indolyl)-propyl]-1-piperazinyl\}-acetylamino)-N-(2-morpholin-4-ylethyl) Benzamide (13h)

5-Bromo-3-(3-piperazin-1-yl-propyl)-1H-indole 9c (187 mg; $0.58 \mathrm{mmol})$, 2-(2-chloro-acetylamino)-5fluoro- $\mathrm{N}$-(2-morpholin-4-yl-ethyl)-benzamide $12 \mathrm{c}(199 \mathrm{mg} ; 0.58 \mathrm{mmol})$, and anhydrous $\mathrm{K}_{2} \mathrm{CO}_{3}(80 \mathrm{mg}$; $0.58 \mathrm{mmol}$ ), to afford $13 \mathrm{~h}$ (303 mg; 85\% yield) as a yellow light solid. m.p.: $73.8-74.7^{\circ} \mathrm{C} ;{ }^{1} \mathrm{H}-\mathrm{NMR}$ (DMSO- $\left.d_{6}\right): \delta 1.71-1.84\left(\mathrm{~m}, 2 \mathrm{H}, \mathrm{H}-2^{\prime}\right), 2.25-2.56\left(\mathrm{~m}, 16 \mathrm{H}, \mathrm{H}-3^{\prime}, \mathrm{H}-4^{\prime}, \mathrm{H}-5^{\prime}, \mathrm{H}-9^{\prime \prime}\right.$, and H-1'"') $2.67(\mathrm{t}, 2 \mathrm{H}$, $\left.\mathrm{H}-1^{\prime}, J=7.2 \mathrm{~Hz}\right), 3.14\left(\mathrm{~s}, 2 \mathrm{H}, \mathrm{H}-6^{\prime}\right), 3.40-3.43\left(\mathrm{~m}, 2 \mathrm{H}, \mathrm{H}-8^{\prime \prime}\right), 3.57$ (t, 4H, H-2'",$\left.J=4.5 \mathrm{~Hz}\right), 7.13-7.27$ (m, $3 \mathrm{H}, \mathrm{H}-2$, and $\mathrm{H}-6$ and H-3" $), 7.31$ (d, 1H, H-7, J = 8.5 Hz), 7.69 (d, 1H, H-4, J = 1.7 Hz), 7.73-7.81 (m, $\left.1 \mathrm{H}, \mathrm{H}-6^{\prime \prime}\right), 7.92\left(\mathrm{dd}, 1 \mathrm{H}, \mathrm{H}-4^{\prime \prime}, J_{o}=6.4 \mathrm{~Hz}, J_{m}=2.7 \mathrm{~Hz}\right), 8.15-8.22\left(\mathrm{~m}, 1 \mathrm{H}, \mathrm{H}-7^{\prime \prime}\right), 9.9$ (br. s, $\left.1 \mathrm{H}, \mathrm{H}-7^{\prime}\right)$, and $11(\mathrm{~s}, 1 \mathrm{H}, \mathrm{H}-1)$ ppm. ${ }^{13} \mathrm{C}-\mathrm{NMR}$ (DMSO- $\left.d_{6}\right)$ : $\delta 22.4,27.5,36.9,52.8(2 \mathrm{X}), 53.3(2 \mathrm{X}), 53.7(2 \mathrm{X}), 57.5$, $57.7,62.2,66.6(2 \mathrm{X}), 111.3,113.8,114.7,115.1\left(\mathrm{~d},{ }^{2} J_{\mathrm{C}-\mathrm{F}}=24.2 \mathrm{~Hz}\right), 118.5\left(\mathrm{~d},{ }^{2} J_{\mathrm{C}-\mathrm{F}}=21.3 \mathrm{~Hz}\right), 121.1,122.6$ $\left(\mathrm{d},{ }^{3} J_{\mathrm{C}-\mathrm{F}}=7.3 \mathrm{~Hz}\right), 123.7,124.3\left(\mathrm{~d},{ }^{3} J_{\mathrm{C}-\mathrm{F}}=5.9 \mathrm{~Hz}\right), 124.4,129.6,134.9\left(\mathrm{~d},{ }^{4} J_{\mathrm{C}-\mathrm{F}}=2.2 \mathrm{~Hz}\right), 135.4,157.3(\mathrm{~d}$, $\left.{ }^{1} J_{\mathrm{C}-\mathrm{F}}=241 \mathrm{~Hz}\right), 166.9$, and $169.5 \mathrm{ppm}$. HRMS: (EI) Calculated for $\mathrm{C}_{30} \mathrm{H}_{38} \mathrm{BrFN}_{6} \mathrm{O}_{3}\left(\mathrm{M}^{+}\right)=629.2251$. Found: 629.2249 . 
4-Fluoro-2-(2-\{4-[3-(1H-3-indolyl)-propyl]-1-piperazinyl\}-acetylamino)-N-(2-morpholin-4-yl-ethyl) Benzamide (13i)

3-(3-Piperazin-1-yl-propyl)-1H-indole 9a (150 mg; $0.62 \mathrm{mmol}), 2$-(2-chloro-acetylamino)-4-fluoro$\mathrm{N}$-(2-morpholin-4-yl-ethyl)-benzamide 12a $(213 \mathrm{mg} ; 0.62 \mathrm{mmol})$, and anhydrous $\mathrm{K}_{2} \mathrm{CO}_{3}(86 \mathrm{mg}$; $0.62 \mathrm{mmol})$, to afford $13 \mathbf{i}\left(249 \mathrm{mg} ; 75 \%\right.$ yield) as a yellow light solid. m.p.: $80.5-81.8^{\circ} \mathrm{C} ;{ }^{1} \mathrm{H}-\mathrm{NMR}$ (DMSO- $\left.d_{6}\right): \delta 1.86\left(\mathrm{~m}, 2 \mathrm{H}, \mathrm{H}-2^{\prime}\right), 2.25-2.49\left(\mathrm{~m}, 12 \mathrm{H}, \mathrm{H}-3^{\prime}, \mathrm{H}-4^{\prime}, \mathrm{H}-9^{\prime \prime}\right.$ and $\left.\mathrm{H}-1^{\prime \prime \prime}\right), 2.58\left(\mathrm{~m}, 4 \mathrm{H}, \mathrm{H}-5^{\prime}\right)$, $2.7\left(\mathrm{t}, 2 \mathrm{H}, \mathrm{H}-1^{\prime}, \mathrm{J}=7.4 \mathrm{~Hz}\right), 3.15\left(\mathrm{~s}, 2 \mathrm{H}, \mathrm{H}-6^{\prime}\right), 3.35-3.40\left(\mathrm{~m}, 2 \mathrm{H}, \mathrm{H}-8^{\prime \prime}\right), 3.54\left(\mathrm{t}, 4 \mathrm{H}, \mathrm{H}-2^{\prime \prime \prime}\right.$, $\left.J=4.3 \mathrm{~Hz}\right)$, 6.93-7.09 (m, 3H, H-5 and H-6 and H-5" ), $7.12(\mathrm{~d}, 1 \mathrm{H}, \mathrm{H}-2, J=1 \mathrm{~Hz}), 7.33(\mathrm{~d}, 1 \mathrm{H}, \mathrm{H}-7, J=8 \mathrm{~Hz}), 7.51(\mathrm{~d}$, $1 \mathrm{H}, \mathrm{H}-4, J=7.8 \mathrm{~Hz}), 7.76\left(\mathrm{dd}, 1 \mathrm{H}, \mathrm{H}-3^{\prime \prime}{ }^{\prime}, J_{o}=8.6 \mathrm{~Hz}, J_{m}=6.7 \mathrm{~Hz}\right), 8.39\left(\mathrm{dd}, 1 \mathrm{H}, \mathrm{H}-6^{\prime \prime}, J_{o}=12.3 \mathrm{~Hz}\right.$, $\left.J_{m}=2.6 \mathrm{~Hz}\right), 8.68\left(\mathrm{t}, 1 \mathrm{H}, \mathrm{H}-7^{\prime \prime}, J=5 \mathrm{~Hz}\right), 10.8(\mathrm{~s}, 1 \mathrm{H}, \mathrm{H}-1)$, and 12.1 (br. s, $\left.1 \mathrm{H}, \mathrm{H}-7^{\prime}\right)$ ppm. ${ }^{13} \mathrm{C}-\mathrm{NMR}$ $\left(\right.$ DMSO- $\left._{6}\right): \delta 22.5,27.7,36.9,52.9(2 X), 53.5(2 X), 53.7(2 X), 55.4,57.6,62.3,66.6(2 X), 107\left(\mathrm{~d},{ }^{2} J_{C-F}=28\right.$ $\mathrm{Hz}), 109.7\left(\mathrm{~d},{ }^{2} J_{\mathrm{C}-\mathrm{F}}=22 \mathrm{~Hz}\right), 111.8,114.5,118.6\left(\mathrm{~d},{ }^{4} J_{\mathrm{C}-\mathrm{F}}=2.9 \mathrm{~Hz}\right), 121.3,122.6,127.6,130.8\left(\mathrm{~d},{ }^{3} J_{\mathrm{C}-\mathrm{F}}=\right.$ $10.9 \mathrm{~Hz}), 134,136.8,140.8\left(\mathrm{~d},{ }^{3} J_{\mathrm{C}-\mathrm{F}}=12.5 \mathrm{~Hz}\right), 141.4,163.8\left(\mathrm{~d},{ }^{1} J_{\mathrm{C}-\mathrm{F}}=245 \mathrm{~Hz}\right), 167.3$, and $170.3 \mathrm{ppm}$. HRMS: (EI) Calculated for $\mathrm{C}_{30} \mathrm{H}_{39} \mathrm{FN}_{6} \mathrm{O}_{3}\left(\mathrm{M}^{+}\right)=551.3145$. Found: 551.3139 .

4-Fluoro-3-(2-\{4-[3-(1H-3-indolyl)-propyl]-1-piperazinyl\}-acetylamino)-N-(2-morpholin-4-yl-ethyl) Benzamide (13j)

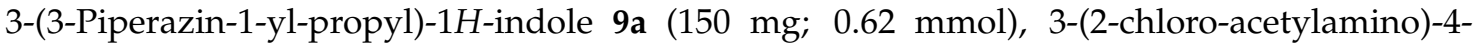
fluoro-N-(2-morpholin-4-yl-ethyl)-benzamide $\mathbf{1 2 b}$ (213 mg; $0.62 \mathrm{mmol})$, and anhydrous $\mathrm{K}_{2} \mathrm{CO}_{3}(86 \mathrm{mg}$; $0.62 \mathrm{mmol})$, to afford $13 \mathrm{j}\left(268 \mathrm{mg} ; 81 \%\right.$ yield) as a yellow light solid. m.p.: $70.2-71.9{ }^{\circ} \mathrm{C} ;{ }^{1} \mathrm{H}-\mathrm{NMR}$ (DMSO- $d_{6}$ ): $\delta 1.81$ (q, 2H, H-2',$\left.J=7.5 \mathrm{~Hz}\right), 2.21-2.53\left(\mathrm{~m}, 16 \mathrm{H}, \mathrm{H}-3^{\prime}, \mathrm{H}-4^{\prime}, \mathrm{H}-5^{\prime}, \mathrm{H}-9^{\prime \prime}\right.$, and H-1'”'), 2.7 (t, 2H, H-1',$J=7.4 \mathrm{~Hz}), 3.19\left(\mathrm{~s}, 2 \mathrm{H}, \mathrm{H}-6^{\prime}\right), 3.38-3.40\left(\mathrm{~m}, 2 \mathrm{H}, \mathrm{H}-8^{\prime \prime}\right), 3.57$ (t, 4H, H-2'",$\left.J=4.5 \mathrm{~Hz}\right), 6.96$ $\left(\mathrm{td}, 1 \mathrm{H}, \mathrm{H}-5\right.$ or H-6, $\left.J_{o}=7.5 \mathrm{~Hz}, J_{m}=1 \mathrm{~Hz}\right), 7.06\left(\mathrm{td}, 1 \mathrm{H}, \mathrm{H}-6\right.$ or H-5, $\left.J_{o}=7.4 \mathrm{~Hz}, J_{m}=1 \mathrm{~Hz}\right), 7.11(\mathrm{~d}$, $1 \mathrm{H}, \mathrm{H}-2, J=2.1 \mathrm{~Hz}), 7.32-7.40\left(\mathrm{~m}, 2 \mathrm{H}, \mathrm{H}-7\right.$ and H-5" $\left.{ }^{\prime \prime}\right), 7.51(\mathrm{~d}, 1 \mathrm{H}, \mathrm{H}-4, J=7.7 \mathrm{~Hz}), 7.61-7.66(\mathrm{~m}, 1 \mathrm{H}$, H-2' $)$, 8.40-8.52 (m, 2H, H-6" and H-7" ), 9.69 (br. s, 1H, H-7'), and 10.8 (s, 1H, H-1) ppm. ${ }^{13} \mathrm{C}-\mathrm{NMR}$ (DMSO- $d_{6}$ ): $\delta 22.4,27.1,36.6,52.8(2 \mathrm{X}), 52.9(2 \mathrm{X}), 53.2(2 \mathrm{X}), 57.2,57.5,61.1,66.2(2 \mathrm{X}), 111.3,114.3,115.1$ $\left(\mathrm{d},{ }^{2} J_{\mathrm{C}-\mathrm{F}}=20.1 \mathrm{~Hz}\right), 118.1\left(\mathrm{~d},{ }^{3} J_{\mathrm{C}-\mathrm{F}}=10.6 \mathrm{~Hz}\right), 120.7,122.1,122.4,123.0,124.0\left(\mathrm{~d},{ }^{3} J_{\mathrm{C}-\mathrm{F}}=8.1 \mathrm{~Hz}\right), 125.7(\mathrm{~d}$, $\left.{ }^{2} J_{\mathrm{C}-\mathrm{F}}=11.8 \mathrm{~Hz}\right), 127.2,131.1\left(\mathrm{~d},{ }^{4} J_{\mathrm{C}-\mathrm{F}}=3.3 \mathrm{~Hz}\right), 136.3,154.7\left(\mathrm{~d},{ }^{1} J_{\mathrm{C}-\mathrm{F}}=249 \mathrm{~Hz}\right), 165.1$, and $168.5 \mathrm{ppm}$. HRMS: (EI) Calculated for $\mathrm{C}_{30} \mathrm{H}_{39} \mathrm{FN}_{6} \mathrm{O}_{3}\left(\mathrm{M}^{+}\right)=551.3145$. Found: 551.3140.

2-Fluoro-5-(2-\{4-[3-(1H-3-indolyl)-propyl]-1-piperazinyl\}-acetylamino)-N-(2-morpholin-4-yl-ethyl) Benzamide (13k)

3-(3-Piperazin-1-yl-propyl)-1H-indole 9a (150 mg; $0.62 \mathrm{mmol})$, 5-(2-chloro-acetylamino)-2fluoro- $N$-(2-morpholin-4-yl-ethyl)-benzamide 12d (213 mg; $0.62 \mathrm{mmol})$, and anhydrous $\mathrm{K}_{2} \mathrm{CO}_{3}(86 \mathrm{mg}$; $0.62 \mathrm{mmol})$, to afford 13k (200 mg; 60.7\% yield) as a yellow light solid. m.p.: 69.7-71.5 ${ }^{\circ} \mathrm{C} ;{ }^{1} \mathrm{H}-\mathrm{NMR}$ $\left(\mathrm{DMSO}-d_{6}\right): \delta 1.81\left(\mathrm{q}, 2 \mathrm{H}, \mathrm{H}-2^{\prime}, J=6.8 \mathrm{~Hz}\right), 2.25-2.54\left(\mathrm{~m}, 16 \mathrm{H}, \mathrm{H}-3^{\prime}, \mathrm{H}-4^{\prime}, \mathrm{H}-5^{\prime}, \mathrm{H}-9^{\prime \prime}\right.$, and H-1'” $), 2.7$

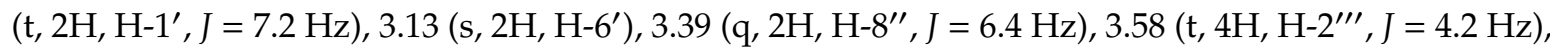
$6.96(\mathrm{t}, 1 \mathrm{H}, \mathrm{H}-5$ or H-6, $J=7.2 \mathrm{~Hz}), 7.06(\mathrm{t}, 1 \mathrm{H}, \mathrm{H}-6$ or H-5, $J=6.9 \mathrm{~Hz}), 7.11(\mathrm{~d}, 1 \mathrm{H}, \mathrm{H}-2, J=1.2 \mathrm{~Hz}), 7.24$ $\left(\mathrm{t}, 1 \mathrm{H}, \mathrm{H}-3^{\prime \prime}, J=9.8 \mathrm{~Hz}\right), 7.34(\mathrm{~d}, 1 \mathrm{H}, \mathrm{H}-4, J=8.0 \mathrm{~Hz}), 7.51(\mathrm{~d}, 1 \mathrm{H}, \mathrm{H}-7, J=7.7 \mathrm{~Hz}), 7.76-7.81(\mathrm{~m}, 1 \mathrm{H}$, H-6" $\left.{ }^{\prime \prime}\right), 7.94\left(\mathrm{dd}, 1 \mathrm{H}, \mathrm{H}-4^{\prime \prime}{ }^{\prime}, J_{o}=6.3 \mathrm{~Hz}, J_{m}=2.6 \mathrm{~Hz}\right), 8.15-8.22\left(\mathrm{~m}, 1 \mathrm{H}, \mathrm{H}-7^{\prime \prime}\right), 9.90$ (br. s, 1H, H-7'), and 10.8 (s, 1H, H-1) ppm. ${ }^{13} \mathrm{C}-\mathrm{NMR}\left(\mathrm{DMSO}_{-}\right)$): $\delta 22.9,27.5,37.0,53.0(2 \mathrm{X}), 53.2(2 \mathrm{X}), 53.7(2 \mathrm{X}), 57.4,57.9$, $62.2,66.7(2 \mathrm{X}), 111.8,114.8,116.7\left(\mathrm{~d},{ }^{2} J_{\mathrm{C}-\mathrm{F}}=24.2 \mathrm{~Hz}\right), 118.5,118.7,121.2,121.4\left(\mathrm{~d},{ }^{4} J_{\mathrm{C}-\mathrm{F}}=2.2 \mathrm{~Hz}\right), 112.6$, $123.6\left(\mathrm{~d},{ }^{3} J_{\mathrm{C}-\mathrm{F}}=8.1 \mathrm{~Hz}\right), 124.1\left(\mathrm{~d},{ }^{2} J_{\mathrm{C}-\mathrm{F}}=16.1 \mathrm{~Hz}\right), 127.7,135.4\left(\mathrm{~d},{ }^{3} J_{\mathrm{C}-\mathrm{F}}=2.9 \mathrm{~Hz}\right), 136.8,155.4\left(\mathrm{~d},{ }^{1} J_{\mathrm{C}-\mathrm{F}}\right.$ $=245 \mathrm{~Hz}), 163.7$, and 168.9 ppm. HRMS: (EI) Calculated for $\mathrm{C}_{30} \mathrm{H}_{39} \mathrm{FN}_{6} \mathrm{O}_{3}\left(\mathrm{M}^{+}\right)=551.3145$. Found: 551.3142 .

5-Fluoro-2-(2-\{4-[3-(1H-3-indolyl)-propyl]-1-piperazinyl\}-acetylamino)-N-(2-morpholin-4-yl-ethyl) Benzamide (131)

3-(3-Piperazin-1-yl-propyl)-1H-indole 9a (200 mg; $0.82 \mathrm{mmol}), 2$-(2-chloro-acetylamino)-5fluoro- $N$-(2-morpholin-4-yl-ethyl)-benzamide 12c (281 mg; $0.82 \mathrm{mmol})$, and anhydrous $\mathrm{K}_{2} \mathrm{CO}_{3}(113 \mathrm{mg}$; 
$0.82 \mathrm{mmol})$, to afford 131 (263 mg; 60\% yield) as a yellow light solid. m.p.: 68.1-69.6 ${ }^{\circ} \mathrm{C} ;{ }^{1} \mathrm{H}-\mathrm{NMR}$

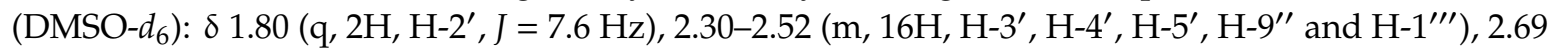
$\left(\mathrm{t}, 2 \mathrm{H}, \mathrm{H}-1^{\prime}, \mathrm{J}=7.4 \mathrm{~Hz}\right), 3.18\left(\mathrm{~s}, 2 \mathrm{H}, \mathrm{H}-6^{\prime}\right), 3.70-3.41\left(\mathrm{~m}, 2 \mathrm{H}, \mathrm{H}-8^{\prime \prime}\right), 3.56\left(\mathrm{t}, 4 \mathrm{H}, \mathrm{H}-2^{\prime \prime \prime}, J=4.5 \mathrm{~Hz}\right), 6.96$ $\left(\mathrm{td}, 1 \mathrm{H}, \mathrm{H}-5\right.$ or H-6, $\left.J_{o}=7.8 \mathrm{~Hz}, J_{m}=1.0 \mathrm{~Hz}\right), 7.05\left(\mathrm{td}, 1 \mathrm{H}, \mathrm{H}-6\right.$ or H-5, $\left.J_{o}=7 \mathrm{~Hz}, \mathrm{~J}_{m}=1.1 \mathrm{~Hz}\right), 7.11(\mathrm{~d}, 1 \mathrm{H}$, $\mathrm{H}-2, J=2.1 \mathrm{~Hz}), 7.33$ (t, 1H, H-4" $\left.{ }^{\prime \prime} J=8.4 \mathrm{~Hz}\right), 7.39$ (d, 1H, H-4, J = 8.7 Hz), 7.50 (d, 1H, H-7, J = 7.8 Hz), 7.59-7.66 (m, 1H, H-3" ), 8.40-8.48 (m, 2H, H-6" and H-7"'), 9.68 (br. s, 1H, H-7'), and 10.75 (s, 1H, H-1) ppm. ${ }^{13}$ C-NMR (DMSO- $\left.d_{6}\right): \delta 22.9,27.7,37.1,53.3(2 \mathrm{X}), 53.4(2 \mathrm{X}), 53.8(2 \mathrm{X}), 57.8,58.1,61.6,66.7(2 \mathrm{X})$, $111.7,113.4,114.9,115.6\left(\mathrm{~d},{ }^{2} J_{\mathrm{C}-\mathrm{F}}=19.4 \mathrm{~Hz}\right), 118.6\left(\mathrm{~d},{ }^{2} J_{\mathrm{C}-\mathrm{F}}=17.7 \mathrm{~Hz}\right), 121.2,122.6,123.0,124.5\left(\mathrm{~d},{ }^{3} J_{\mathrm{C}-\mathrm{F}}\right.$ $=8.3 \mathrm{~Hz}), 126.2\left(\mathrm{~d},{ }^{3} J_{\mathrm{C}-\mathrm{F}}=11.6 \mathrm{~Hz}\right), 127.7,131.63\left(\mathrm{~d},{ }^{4} J_{\mathrm{C}-\mathrm{F}}=2.8 \mathrm{~Hz}\right), 136.7,155.3\left(\mathrm{~d},{ }^{1} J_{\mathrm{C}-\mathrm{F}}=249 \mathrm{~Hz}\right)$, 165.5, and 169.1 ppm. HRMS: (EI) Calculated for $\mathrm{C}_{30} \mathrm{H}_{39} \mathrm{FN}_{6} \mathrm{O}_{3}\left(\mathrm{M}^{+}\right)=551.3145$. Found: 551.3141.

\subsection{Biological Assay}

\subsubsection{Reagents}

$\left[{ }^{3} \mathrm{H}\right]$ Paroxetine (20.8 Ci/mmol; Code NET869), [ $\left.{ }^{3} \mathrm{H}\right]-$ Methylspiperone (specific activity 64.1 $\mathrm{Ci} / \mathrm{mmol}$; NET856), membrane from clonal cell line HEK-293 that overexpresses SERT (Code: RBHSTM400UA), and membrane from CHO-K1 clonal cell line that overexpresses $\mathrm{D}_{2}$ receptor (Code: RBHD2CM400UA) were purchased from Perkin-Elmer (Boston, MA, USA). Fluoxetine and haloperidol were purchased from Sigma-Aldrich (St. Louis, MO, USA). All other reagents used were of analytical grade.

\subsubsection{SERT Binding}

To determine the binding of all compounds at SERT, competitive binding assays were performed according to previously reported procedures with some modifications [36]. Briefly, assays were carried out in a total volume of $0.5 \mathrm{~mL}$ containing $9 \mu \mathrm{g}$ protein of membrane from a clonal cell line HEK-293 that overexpresses SERT, $50 \mathrm{mM}$ Tris buffer, $\mathrm{pH}$ 7.4, $120 \mathrm{mM} \mathrm{NaCl}, 5 \mathrm{mM} \mathrm{KCl}, 2 \mathrm{nM}\left[{ }^{3} \mathrm{H}\right]$-paroxetine (specific activity $20.8 \mathrm{Ci} / \mathrm{mmol}$, PerkinElmer), and the compounds to be tested at different concentrations $\left(10^{-9}-10^{-4} \mathrm{M}\right)$. After $1 \mathrm{~h}$ at $27^{\circ} \mathrm{C}$, incubations were stopped by rapid filtration through Whatman $\mathrm{GF} / \mathrm{C}$ filters presoaked in $0.5 \%$ polyethyleneimine, which were washed five times with $3 \mathrm{~mL}$ of ice-cold buffer, dried, and put in Eppendorf tubes with scintillation liquid. Radioactivity was counted by a liquid scintillation counter (MicroBeta 2450 microplate counter, PerkinElmer). Control curve was performed with fluoxetine in the same experimental conditions. Non-specific binding was determined with $10 \mu \mathrm{M}$ fluoxetine.

\subsection{3. $\mathrm{D}_{2}$ Receptor Binding}

To determine the binding of all compounds at $\mathrm{D}_{2}$ receptor, competitive binding assays were performed according to provider indications with some modifications. Briefly, assays were carried out in a total volume of $0.5 \mathrm{~mL}$ containing $3 \mu \mathrm{g}$ protein of membrane from a $\mathrm{CHO}-\mathrm{K} 1$ clonal cell line that overexpresses $\mathrm{D}_{2}$ receptor, $50 \mathrm{mM}$ Tris buffer, $\mathrm{pH}$ 7.4, $120 \mathrm{mM} \mathrm{NaCl}, 5 \mathrm{mM} \mathrm{KCl}, 5 \mathrm{mM} \mathrm{MgCl} 2,1 \mathrm{mM}$ EDTA, $0.5 \mathrm{nM}\left[{ }^{3} \mathrm{H}\right]$-methylspiperone (specific activity $64.1 \mathrm{Ci} / \mathrm{mmol}$, PerkinElmer), and the compounds to be tested at different concentrations $\left(10^{-9}-10^{-4} \mathrm{M}\right)$. After $2 \mathrm{~h}$ at $27^{\circ} \mathrm{C}$, incubations were stopped by rapid filtration through Whatman GF/C filters presoaked in $0.5 \%$ polyethyleneimine, which were washed five times with $3 \mathrm{~mL}$ of ice-cold wash buffer ( $50 \mathrm{mM}$ Tris buffer, $\mathrm{pH} 7.4,154 \mathrm{mM} \mathrm{NaCl})$, dried, and put in Eppendorf tubes with scintillation liquid. Radioactivity was counted as described before. Control curve was performed with haloperidol in the same conditions. Non-specific binding was determined with $10 \mu \mathrm{M}$ haloperidol.

Analysis of data: All curves were fitted using the sigmoidal dose-response inhibition curve (variable slope) equation built into GraphPad PRISM 5.01 (GraphPad Software Inc., San Diego, CA, USA). The analysis gives the $\mathrm{IC}_{50}$ value (i.e., the drug concentration inhibiting specific binding by $50 \%$ ) to calculate $\mathrm{Ki}$ (affinity constant) by the Cheng-Prussof equation $\left(\mathrm{Ki}=\mathrm{IC}_{50} /(1+([\right.$ radioligand] $/ \mathrm{Kd}$ 
(radioligand))). The Kd values used correspond to $0.13 \mathrm{nM}$ to [ ${ }^{3} \mathrm{H}$ ] paroxetine on SERT [50], and 0.1 $\mathrm{nM}$ to $\left[{ }^{3} \mathrm{H}\right]$-methylspiperone on $\mathrm{D}_{2}$ (provided by the manufacturer). The $\mathrm{IC}_{50}$ and $\mathrm{Ki}$ values correspond to the results of three independent experiments, each in triplicate. All data are expressed as the mean \pm SEM.

\subsubsection{MAO-A Inhibition}

All experimental procedures were approved by the Ethics Committee of the University of Santiago de Chile and the Science Council (FONDECYT) of Chile and followed internationally accepted guidelines (NIH Guide for the Care and Use of Laboratory Animals).

The effects of the compounds on rat MAO-A activity were studied following a previously reported methodology $[50,51]$, using a crude rat brain mitochondrial suspension as a source of enzyme. Serotonin $(100 \mu \mathrm{M})$ was used as the selective substrate for MAO-A. This compound and its metabolite were detected by HPLC with electrochemical detection. As an exploratory evaluation, the percentage of MAO-A inhibition in the presence of $100 \mu \mathrm{M}$ of the different compounds was determined, with the idea of evaluating in detail those compounds showing an inhibitory activity in the range of 70-100\%.

\subsubsection{Molecular Docking}

Molecular docking studies for the two families of compounds were performed on two different protein targets (SERT and $\mathrm{D}_{2}$ receptor). All dockings were carried out at $\mathrm{pH} 7.4$ in the crystal structures of human SERT (hSERT PDB: 5I73) [47] and human $\mathrm{D}_{2}$ receptor [hD 2 PDB: 6CM4) [49]. All compounds were modelled using the Spartan'14 Software (Wavefunction, Inc. Irvine, CA) and geometry optimization calculations were carried out using the software package at the Hartree-Fock level using the 6-31G* basis set. Docking studies were performed using AutoDockv4.2 [52] software suite with Autodock Tools ADT 1.5.6 [52,53] following the standard docking procedure for rigid proteins. Grid maps were calculated using the autogrid option with a grid volume of $70 \times 70 \times 70$ points with a grid spacing of $0.375 \AA$ and centered on the coordinates $x, y, z: 33.3184 .5 ; 0.565-9.397$; and 37.019 28.136 for the SERT and $\mathrm{D}_{2}$ receptor respectively. Docking simulations were performed with a Lamarckian genetic algorithm (LGA) and binding energies were estimated according to the internal scoring function implemented by the program; 250 independent runs per ligand were carried out with an initial population of 300 individuals. Default settings were used for all other parameters. The lowest free-energy resulting complexes were selected and further analyzed using the Visual Molecular Dynamic (VMD) visualization program [54]. Validation of the docking protocol was performed using the co-crystallized ligands $(S)$-citalopram and risperidone for SERT and $\mathrm{D}_{2}$, respectively.

\subsubsection{QSAR Methods}

CoMFA and CoMSIA studies were performed with Sybyl X-1.2 software [55] installed in a Windows 10 environment on a PC with an Intel Core i7 CPU. The geometric optimization, field calculation, and charges calculation were performed as previously reported [56] (Figure S1 and Table S3 in Supplementary Material) [57]. The internal validation of the models was done by calculating the cross-validation coefficient $\mathrm{q}^{2}$ [58]. The models with the highest value of $\mathrm{q}^{2}$ were selected and then subjected to external validation [59-61] (Table S2). In all cases, the best models passed the validation limits [59] (Table S2). The regression graphs of each model and the tables of experimental versus calculated values are in the Supplementary Material (Table S3, Figure S2).

\section{Conclusions}

According to these results, the design of hybrid or bifunctional compounds, i.e., molecules that incorporate two pharmacophores known to act at different receptors into a single chemical entity, is an attractive approach for the development of agents having a targeted polypharmacological profile $[19,62,63]$. In the present work, we attempted to combine SERT effects previously demonstrated for indolylalkylpiperazine derivatives, functionalizing the parent scaffold with structural fragments of 
drugs with known activity upon $\mathrm{D}_{2}$ receptor or MAO-A. Unexpectedly, the synthesized compounds did not show, in most cases, a multitarget profile, since they exhibited a high affinity for SERT while showing almost no effect at $\mathrm{D}_{2}$ receptor or MAO-A. This indicates that this strategy, although plausible, requires a very fine design of the fragments to be connected and how these are going to be linked. Beyond these considerations, our results highlight the remarkable stability of the indolylpropylpiperazine skeleton as SERT ligand, which exhibits a high affinity by this target, apparently regardless of the type of the associated moiety [34-36]. We think that this represents an important feature for the design of polypharmacological molecules, in which an effect upon SERT is pursued. Docking and QSAR results allowed us to rationalize the high SERT affinity observed for compounds in both studied families. Thus, the presence of a halogen at the C-5 position of the indole ring and fluorine atoms at the benzoxazine (Series I) or acetanilide (Series II) moieties probably induces electronic deprotection of the corresponding aromatic rings, favoring stronger $\pi-\pi$ interactions of these frameworks with donor aromatic residues at the binding site.

Interestingly, one of the compounds (7n) showed a promissory multitarget profile, being the only derivative showing a relatively high and comparable affinity for SERT and $\mathrm{D}_{2}$ receptor $(K \mathrm{i}=84.4$ and $307 \mathrm{nM}$, respectively). Even though at this time it is difficult to determine the molecular aspects underlying this pharmacological promiscuity, it is clear that for polypharmacological drugs, a similar affinity for different receptors is the most relevant characteristic, and therefore $\mathbf{7 n}$ stands as a very attractive lead for further optimization.

Supplementary Materials: Supplementary materials are available online. Table S1. Statistical parameters and Field combinations for CoMFA and CoMSIA. Table S2. Summary of external validation parameters for CoMFA and CoMSIA. Table S3. Experimental and predicted pKi and residual values for analyzed compounds according to CoMFA and CoMSIA. Figure S1. The superimposed structures of all compounds used in the CoMFA/CoMSIA models. Figure S2. Plots of experimental versus predicted $\mathrm{pKi}$ values for the training and test set molecules for CoMFA (A, B) and CoMSIA (C, D) models. Figure S3. hSERT affinity curves for compounds of Series I (7a, 7b, $\mathbf{7 c}, 7 \mathrm{~d}, 7 \mathbf{e}, 7 \mathbf{f}, 7 \mathrm{~g}, 7 \mathbf{h}, 7 \mathbf{i}, 7 \mathbf{j}, 7 \mathbf{k}, 7 \mathbf{7}, \mathbf{7 m}, \mathbf{7 n}, \mathbf{7 o}$, and fluoxetine), displaying $\mathrm{IC}_{50}$ values. Each determination was made in triplicate and the data were expressed as the mean \pm SD. Figure S4. D2 affinity curves for compounds of Series I $\left(\mathbf{7 a}, 7 \mathbf{b}, 7 \mathbf{c}, 7 \mathbf{d}, 7 \mathbf{e}, 7 \mathbf{f}, 7 \mathbf{g}, 7 \mathbf{h}, 7 \mathbf{i}, 7 \mathbf{j}, 7 \mathbf{k}, 7 \mathbf{7}, 7 \mathbf{m}, \mathbf{7 n}, \mathbf{7 o}\right.$, and haloperidol), displaying $\mathrm{IC}_{50}$ values. Each determination was made in triplicate and the data were expressed as the mean \pm SD. Figure S5. hSERT affinity curves for compounds of Series II (13a, 13b, 13c, 13d, 13e, 13f, 13g, 13h, 13i, 13j, 13k, 13l, and fluoxetine), displaying $\mathrm{IC}_{50}$ values. Each determination was made in triplicate and the data were expressed as the mean $\pm \mathrm{SD}$. Figure S6. D2 affinity curves for compounds of Series II (13a, 13b, 13c, 13d, 13e, 13f, 13g, 13h, 13i, 13j, 13k, 131, and haloperidol), displaying $\mathrm{IC}_{50}$ values. Each determination was made in triplicate and the data expressed as the mean \pm SD.

Author Contributions: Conceptualization, H.P.-M. and M.R.-P.; methodology, C.C.-C., J.R.-L., J.A.-E., C.S., C.O.-G., G.Q. and P.I.-V.; software, J.M.-R., D.C., C.D.P.-M. and H.C.; validation, H.P.-M., R.A.-M. and M.R.-P.; formal analysis, H.P.-M., C.C.-C., R.A.-M., C.S., P.I.-V., J.M.-R., C.D.P.-M., H.C. and M.R.-P.; resources, H.P.-M., J.M.-R., P.I.-V. and M.R.-P.; data curation, H.P.-M., C.C.-C., J.M.-R., H.C. and M.R.-P.; writing-original draft preparation, H.P.-M., C.C.-C. and M.R.-P.; writing-review and editing, H.P.-M., J.R.-L., J.A.-E., J.M.-R., H.C. and M.R.-P.; supervision, H.P.-M. and M.R.-P.; project administration, H.P.-M., J.M.-R., P.I.-V. and M.R.-P.; funding acquisition, H.P.-M., P.I.-V. and M.R.-P. All authors have read and agreed to the published version of the manuscript.

Funding: This research was funded by FONDECYT (Chile) Grant numbers: 1170269 (H. Pessoa-Mahana), 1170662 (M. Reyes-Parada) and FONDECYT Postdoctoral Grant 3180602 (J. Alarcón-Espósito). G. Quiroz was fellow of the CONICYT National Ph.D. Scholar Fellowship.

Acknowledgments: The authors are grateful to Claudia Sanhueza for her valuable technical assistance. Funding: This work was supported by FONDECYT Grant numbers: 1170269 (H. Pessoa-Mahana), 1170662 (M. Reyes-Parada); FONDECYT Postdoctoral Grant 3180602 (J. Alarcón-Espósito). G. Quiroz was fellow of the CONICYT National Ph.D. Scholar Fellowship.

Conflicts of Interest: The authors declare no conflict of interest.

\section{References}

1. Depression Fact Sheet; World Health Organization (WHO): Geneva, Switzerland, 2020; Available online: https://www.who.int/news-room/fact-sheets/detail/depression (accessed on 10 September 2020).

2. Miller, N.; Perich, T.; Meade, T. Depression, mania and self-reported creativity in bipolar disorder. Psychiatry Res. 2019, 276, 129-133. [CrossRef] [PubMed] 
3. Watters, A.J.; Carpenter, J.S.; Harris, A.W.F.; Korgaonkar, M.S.; Williams, L.M. Characterizing neurocognitive markers of familial risk for depression using multi-modal imaging, behavioral and self-report measures. J. Affect. Disord. 2019, 253, 336-342. [CrossRef] [PubMed]

4. McIntosh, A.M.; Sullivan, P.F.; Lewis, C.M. Uncovering the Genetic Architecture of Major Depression. Neuron 2019, 102, 91-103. [CrossRef]

5. Coretti, S.; Rumi, F.; Cicchetti, A. The Social Cost of Major Depression. A Systematic Review. Rev. Eur. Stud. 2019, 11, 73. [CrossRef]

6. Collins, P.Y.; Patel, V.; Joestl, S.S.; March, D.; Insel, T.R.; Daar, A.S.; Bordin, I.A.; Costello, E.J.; Durkin, M.; Fairburn, C.; et al. Grand challenges in global mental health. Nature 2011, 475, 27-30. [CrossRef]

7. Hirschfeld, R.M. History and evolution of the monoamine hypothesis of depression. J. Clin. Psychiatry 2000, 61 (Suppl. 6), 4-6.

8. Berton, O.; Nestler, E.J. New approaches to antidepressant drug discovery: Beyond monoamines. Nat. Rev. Neurosci. 2006, 7, 137-151. [CrossRef]

9. Hasler, G. Pathophysiology of depression: Do we have any solid evidence of interest to clinicians? World Psychiatry 2010, 9, 155-161. [CrossRef]

10. Lazar, M.A.; McIntyre, R.S. Chapter 34 Novel Therapeutic Targets for Major Depressive Disorder. In Neurobiology of Depression; Academic Press: London, UK, 2019; pp. 383-400.

11. Hendrie, C.; Pickles, A. The failure of the antidepressant drug discovery process is systemic. J. Psychopharmacol. 2013, 27, 407-416. [CrossRef]

12. Brummelte, S.; Mc Glanaghy, E.; Bonnin, A.; Oberlander, T.F. Developmental changes in serotonin signaling: Implications for early brain function, behavior and adaptation. Neuroscience 2017, 342, 212-231. [CrossRef]

13. David, D.J.; Gardier, A.M. The pharmacological basis of the serotonin system: Application to antidepressant response. L'Encephale 2016, 42, 255-263. [CrossRef] [PubMed]

14. Javelot, H. Psychopharmacology of anxiety and depression: Historical aspects, current treatments and perspectives. Ann. Pharm. Fr. 2016, 74, 93-118. [CrossRef] [PubMed]

15. Daut, R.A.; Fonken, L.K. Circadian regulation of depression: A role for serotonin. Front. Neuroendocrinol. 2019, 54, 100746. [CrossRef] [PubMed]

16. Hillhouse, T.M.; Porter, J.H. A brief history of the development of antidepressant drugs: From monoamines to glutamate. Exp. Clin. Psychopharmacol. 2015, 23, 1-21. [CrossRef]

17. Milelli, A.; Turrini, E.; Catanzaro, E.; Maffei, F.; Fimognari, C. Perspectives in Designing Multifunctional Molecules in Antipsychotic Drug Discovery. Drug Dev. Res. 2016, 77, 437-443. [CrossRef]

18. Mendonça Júnior, F.J.B.; Scotti, L.; Ishiki, H.; Botelho, S.P.S.; Da Silva, M.S.; Scotti, M.T. Benzo- and thienobenzo- diazepines: Multi-target drugs for CNS disorders. Mini Rev. Med. Chem. 2015, 15, 630-647. [CrossRef]

19. Reyes-Parada, M.; Iturriaga-Vasquez, P. The development of novel polypharmacological agents targeting the multiple binding sites of nicotinic acetylcholine receptors. Expert Opin. Drug Discov. 2016, 11, 969-981. [CrossRef]

20. Subbaiah, M.A.M. Triple Reuptake Inhibitors as Potential Therapeutics for Depression and Other Disorders: Design Paradigm and Developmental Challenges. J. Med. Chem. 2018, 61, 2133-2165. [CrossRef]

21. Juárez Olguín, H.; Calderón Guzmán, D.; Hernández García, E.; Barragán Mejía, G. The Role of Dopamine and Its Dysfunction as a Consequence of Oxidative Stress. Oxid. Med. Cell. Longev. 2016, 2016, 9730467. [CrossRef]

22. Berridge, K.C. The debate over dopamine's role in reward: The case for incentive salience. Psychopharmacology 2007, 191, 391-431. [CrossRef]

23. Wise, R.A. Dopamine, learning and motivation. Nat. Rev. Neurosci. 2004, 5, 483-494. [CrossRef] [PubMed]

24. Belujon, P.; Grace, A.A. Dopamine System Dysregulation in Major Depressive Disorders. Int. J. Neuropsychopharmacol. 2017, 20, 1036-1046. [CrossRef] [PubMed]

25. Brisch, R.; Saniotis, A.; Wolf, R.; Bielau, H.; Bernstein, H.-G.; Steiner, J.; Bogerts, B.; Braun, K.; Jankowski, Z.; Kumaratilake, J.; et al. The Role of Dopamine in Schizophrenia from a Neurobiological and Evolutionary Perspective: Old Fashioned, but Still in Vogue. Front. Psychiatry 2014, 5, 47. [CrossRef] [PubMed]

26. Meder, D.; Herz, D.M.; Rowe, J.B.; Lehéricy, S.; Siebner, H.R. The role of dopamine in the brain-Lessons learned from Parkinson's disease. Neuroimage 2019, 190, 79-93. [CrossRef] [PubMed] 
27. Dunlop, B.W.; Nemeroff, C.B. The Role of Dopamine in the Pathophysiology of Depression. Arch. Gen. Psychiatry 2007, 64, 327-337. [CrossRef]

28. Gold, M.S.; Blum, K.; Febo, M.; Baron, D.; Modestino, E.J.; Elman, I.; Badgaiyan, R.D. Molecular role of dopamine in anhedonia linked to reward deficiency syndrome (RDS) and anti- reward systems. Front. Biosci. (Schol. Ed.) 2018, 10, 309-325. [CrossRef]

29. Cramer, A.O.J.; van Borkulo, C.D.; Giltay, E.J.; van der Maas, H.L.J.; Kendler, K.S.; Scheffer, M.; Borsboom, D. Major Depression as a Complex Dynamic System. PLoS ONE 2016, 11, e0167490. [CrossRef]

30. Liu, W.; Wang, H.; Li, X.; Xu, Y.; Zhang, J.; Wang, W.; Gong, Q.; Qiu, X.; Zhu, J.; Mao, F.; et al. Design, synthesis and evaluation of vilazodone-tacrine hybrids as multitarget-directed ligands against depression with cognitive impairment. Bioorg. Med. Chem. 2018, 26, 3117-3125. [CrossRef]

31. Dessalew, N.; Mikre, W.; Hymete, A. Multitarget Selective Antidepressants Design: Latest Developments, Opportunities and Challenges. Cent. Nerv. Syst. Agents Med. Chem. 2008, 8, 131-142. [CrossRef]

32. Millan, M.J. On "polypharmacy" and multi-target agents, complementary strategies for improving the treatment of depression: A comparative appraisal. Int. J. Neuropsychopharmacol. 2014, 17, 1009-1037. [CrossRef]

33. Rodríguez-Lavado, J.; Gallardo-Garrido, C.; Mallea, M.; Bustos, V.; Osorio, R.; Hödar-Salazar, M.; Chung, H.; Araya-Maturana, R.; Lorca, M.; Pessoa-Mahana, C.D.; et al. Synthesis, in vitro evaluation and molecular docking of a new class of indolylpropyl benzamidopiperazines as dual AChE and SERT ligands for Alzheimer's disease. Eur. J. Med. Chem. 2020, 198, 112368. [CrossRef] [PubMed]

34. Pessoa-Mahana, H.; Silva-Matus, P.; Pessoa-Mahana, C.D.; Chung, H.; Iturriaga-Vásquez, P.; Quiroz, G.; Möller-Acuña, P.; Zapata-Torres, G.; Saitz-Barría, C.; Araya-Maturana, R.; et al. Synthesis and Docking of Novel 3-Indolylpropyl Derivatives as New Polypharmacological Agents Displaying Affinity for 5-HT(1A) R/SERT. Arch. Pharm. (Weinh.) 2017, 350. [CrossRef]

35. Pessoa-Mahana, H.; González-Lira, C.; Fierro, A.; Zapata-Torres, G.; Pessoa-Mahana, C.D.; Ortiz-Severin, J.; Iturriaga-Vásquez, P.; Reyes-Parada, M.; Silva-Matus, P.; Saitz-Barría, C.; et al. Synthesis, docking and pharmacological evaluation of novel homo- and hetero-bis 3-piperazinylpropylindole derivatives at SERT and 5-HT1A receptor. Bioorg. Med. Chem. 2013, 21, 7604-7611. [CrossRef] [PubMed]

36. Ojeda-Gómez, C.; Pessoa-Mahana, H.; Iturriaga-Vásquez, P.; Pessoa-Mahana, C.D.; Recabarren-Gajardo, G.; Méndez-Rojas, C. Synthesis and biological screening of novel indolalkyl arenes targeting the serotonine transporter. Arch. Pharm. (Weinh.) 2014, 347, 174-184. [CrossRef]

37. Pessoa-Mahana, H.; Núñez, C.U.; Araya-Maturana, R.; Barría, C.S.; Zapata-Torres, G.; Pessoa-Mahana, C.D.; Iturriaga-Vasquez, P.; Mella-Raipán, J.; Reyes-Parada, M.; Celis-Barros, C. Synthesis, 5-hydroxytryptamine1A receptor affinity and docking studies of 3-[3-(4-aryl-1-piperazinyl)-propyl]-1H-indole derivatives. Chem. Pharm. Bull. (Tokyo) 2012, 60, 632-638. [CrossRef]

38. Prins, L.H.A.; Petzer, J.P.; Malan, S.F. Inhibition of monoamine oxidase by indole and benzofuran derivatives. Eur. J. Med. Chem. 2010, 45, 4458-4466. [CrossRef]

39. Heinrich, T.; Böttcher, H.; Gericke, R.; Bartoszyk, G.D.; Anzali, S.; Seyfried, C.A.; Greiner, H.E.; Van Amsterdam, C. Synthesis and structure-activity relationship in a class of indolebutylpiperazines as dual 5-HT(1A) receptor agonists and serotonin reuptake inhibitors. J. Med. Chem. 2004, 47, 4684-4692. [CrossRef]

40. Mathew, B.; Baek, S.C.; Thomas Parambi, D.G.; Lee, J.P.; Mathew, G.E.; Jayanthi, S.; Vinod, D.; Rapheal, C.; Devikrishna, V.; Kondarath, S.S.; et al. Potent and highly selective dual-targeting monoamine oxidase-B inhibitors: Fluorinated chalcones of morpholine versus imidazole. Arch. Pharm. (Weinh.) 2019, 352, 1800309. [CrossRef]

41. Weichert, D.; Stanek, M.; Hübner, H.; Gmeiner, P. Structure-guided development of dual $\beta 2$ adrenergic/dopamine D2 receptor agonists. Bioorg. Med. Chem. 2016, 24, 2641-2653. [CrossRef]

42. Männel, B.; Hübner, H.; Möller, D.; Gmeiner, P. $\beta$-Arrestin biased dopamine D2 receptor partial agonists: Synthesis and pharmacological evaluation. Bioorg. Med. Chem. 2017, 25, 5613-5628. [CrossRef]

43. Szabo, M.; Lim, H.D.; Herenbrink, C.K.; Christopoulos, A.; Lane, J.R.; Capuano, B. Proof of concept study for designed multiple ligands targeting the dopamine D2, serotonin 5-HT2A, and muscarinic M1 acetylcholine receptors. J. Med. Chem. 2015, 58, 1550-1555. [CrossRef] [PubMed]

44. Ghanbarpour, A.; Hadizadeh, F.; Piri, F.; Rashidi-Ranjbar, P. Synthesis, conformational analysis and antidepressant activity of moclobemide new analogues. Pharm. Acta Helv. 1997, 72, 119-122. [CrossRef] 
45. Bonnet, U. Moclobemide: Therapeutic use and clinical studies. Cns Drug Rev. 2003, 9, 97-140. [CrossRef] [PubMed]

46. Méndez-Rojas, C.; Quiroz, G.; Faúndez, M.; Gallardo-Garrido, C.; Pessoa-Mahana, C.D.; Chung, H.; Gallardo-Toledo, E.; Saitz-Barría, C.; Araya-Maturana, R.; Kogan, M.J.; et al. Synthesis and biological evaluation of potential acetylcholinesterase inhibitors based on a benzoxazine core. Arch. Pharm. (Weinh.) 2018, 351, 1800024. [CrossRef] [PubMed]

47. Coleman, J.A.; Green, E.M.; Gouaux, E. X-ray structures and mechanism of the human serotonin transporter. Nature 2016, 532, 334-339. [CrossRef]

48. Wang, H.; Goehring, A.; Wang, K.H.; Penmatsa, A.; Ressler, R.; Gouaux, E. Structural basis for action by diverse antidepressants on biogenic amine transporters. Nature 2013, 503, 141-145. [CrossRef]

49. Wang, S.; Che, T.; Levit, A.; Shoichet, B.K.; Wacker, D.; Roth, B.L. Structure of the D2 dopamine receptor bound to the atypical antipsychotic drug risperidone. Nature 2018, 555, 269-273. [CrossRef]

50. Scorza, M.C.; Carrau, C.; Silveira, R.; Zapata-Torres, G.; Cassels, B.K.; Reyes-Parada, M. Monoamine Oxidase Inhibitory Properties of Some Methoxylated and Alkylthio Amphetamine Derivatives: Structure-Activity Relationships. Biochem. Pharmacol. 1997, 54, 1361-1369. [CrossRef]

51. Tatsumi, M.; Groshan, K.; Blakely, R.D.; Richelson, E. Pharmacological profile of antidepressants and related compounds at human monoamine transporters. Eur. J. Pharmacol. 1997, 340, 249-258. [CrossRef]

52. Morris, G.M.; Huey, R.; Lindstrom, W.; Sanner, M.F.; Belew, R.K.; Goodsell, D.S.; Olson, A.J. AutoDock4 and AutoDockTools4: Automated docking with selective receptor flexibility. J. Comput. Chem. 2009, 30, 2785-2791. [CrossRef]

53. Sanner, M.F. Python: A programming language for software integration and development. J. Mol. Graph. Model. 1999, 17, 57-61. [PubMed]

54. Humphrey, W.; Dalke, A.; Schulten, K. VMD: Visual molecular dynamics. J. Mol. Graph. 1996, 14, 33-38. [CrossRef]

55. SYBYL-X 1.2; Tripos International: St. Louis, MO, USA, 2010.

56. Lorca, M.; Valdes, Y.; Chung, H.; Romero-Parra, J.; Pessoa-Mahana, C.D.; Mella, J. Three-Dimensional Quantitative Structure-Activity Relationships (3D-QSAR) on a Series of Piperazine-Carboxamides Fatty Acid Amide Hydrolase (FAAH) Inhibitors as a Useful Tool for the Design of New Cannabinoid Ligands. Int. J. Mol. Sci. 2019, 20, 2510. [CrossRef] [PubMed]

57. Klebe, G.; Abraham, U.; Mietzner, T. Molecular similarity indices in a comparative analysis (CoMSIA) of drug molecules to correlate and predict their biological activity. J. Med. Chem. 1994, 37, 4130-4146. [CrossRef] [PubMed]

58. Clark, M.; Cramer III, R.D.; Van Opdenbosch, N. Validation of the general purpose tripos 5.2 force field. J. Comput. Chem. 1989, 10, 982-1012. [CrossRef]

59. Golbraikh, A.; Tropsha, A. Beware of q2! J. Mol. Graph. Model. 2002, 20, 269-276. [CrossRef]

60. Tropsha, A. Best Practices for QSAR Model Development, Validation, and Exploitation. Mol. Inform. 2010, 29, 476-488. [CrossRef]

61. Roy, K.; Chakraborty, P.; Mitra, I.; Ojha, P.K.; Kar, S.; Das, R.N. Some case studies on application of "r(m)2" metrics for judging quality of quantitative structure-activity relationship predictions: Emphasis on scaling of response data. J. Comput. Chem. 2013, 34, 1071-1082. [CrossRef]

62. Butini, S.; Nikolic, K.; Kassel, S.; Brückmann, H.; Filipic, S.; Agbaba, D.; Gemma, S.; Brogi, S.; Brindisi, M.; Campiani, G.; et al. Polypharmacology of dopamine receptor ligands. Prog. Neurobiol. 2016, 142, 68-103. [CrossRef]

63. Turnaturi, R.; Aricò, G.; Ronsisvalle, G.; Parenti, C.; Pasquinucci, L. Multitarget opioid ligands in pain relief: New players in an old game. Eur. J. Med. Chem. 2016, 108, 211-228. [CrossRef]

Sample Availability: Samples of the compounds are available from the authors. 